University of Tennessee Health Science Center

UTHSC Digital Commons

\title{
Inflammatory Proteins, Genetic Variation, and Environmental Influences on Health Care Associated Infection Development in Sepsis
}

Reba Antoinette Umberger

University of Tennessee Health Science Center

Follow this and additional works at: https://dc.uthsc.edu/dissertations

Part of the Amino Acids, Peptides, and Proteins Commons, and the Bacterial Infections and Mycoses Commons

\section{Recommended Citation}

Umberger, Reba Antoinette, "Inflammatory Proteins, Genetic Variation, and Environmental Influences on Health Care Associated Infection Development in Sepsis" (2011). Theses and Dissertations (ETD). Paper 279. http://dx.doi.org/10.21007/etd.cghs.2011.0330.

This Dissertation is brought to you for free and open access by the College of Graduate Health Sciences at UTHSC Digital Commons. It has been accepted for inclusion in Theses and Dissertations (ETD) by an authorized administrator of UTHSC Digital Commons. For more information, please contact jwelch30@uthsc.edu. 


\title{
Inflammatory Proteins, Genetic Variation, and Environmental Influences on Health Care Associated Infection Development in Sepsis
}

\begin{abstract}
The purpose of this study was to determine the impact of baseline systemic inflammation (pro-inflammatory cytokine, anti-inflammatory cytokine, and their ratio), genetic variability, and environment on the development of health care associated infections (HAI) among sepsis patients during their ICU stay (up to 28 days).

Methods: A prospective observation study was conducted at the Veterans Affairs Medical Center in the Medical Intensive Care Unit over an 18 month period. A total of 78 patients were enrolled within 72 hours of presenting to the ICU with sepsis. Patient were excluded if they were receiving immunosuppressants (chemotherapy or greater than one $\mathrm{mg} / \mathrm{kg}$ of prednisone or equivalent dose), immunosuppressed (AIDS, cancer), or had liver failure (Child Pugh category $\mathrm{C}$ or higher). Baseline plasma and buccal swabs were collected. Patients were followed prospectively through their ICU stay (or for a maximum of 28 days) for the development of HAI as defined by CDC guidelines. Primary variables included baseline IL- 6 and IL-10 levels, IL-6 SNP rs1800795, IL-10 SNP rs1800896, APACHE II, invasive devices, and development of HAI.
\end{abstract}

Results: A total of $17 \mathrm{HAl}$ were identified with $64 \%$ caused by Candida. There were no significant differences in levels of pro-inflammatory cytokine, anti-inflammatory cytokine, or their ratio among subjects who did and did not develop at least one HAI during their ICU stay. There were also no significant differences in rs 1800795 or rs1800896 genotypes for those who did and did not develop HAl; however, racial differences were detected in genotypes among white and black patients with sepsis who did and did not develop HAI. There was a significant difference in rs 1800795 genotype among black patients with sepsis who did not develop HAl compared to whites patients with sepsis who did not develop HAI $(\mathrm{p}=$ 0.006). Specifically, black patients had a lower CG (17.4\% vs. $42.1 \%)$ and higher GG ( $82.6 \%$ vs. $42.1 \%)$ than white patients. There were no racial differences when comparing white and black sepsis patients who developed $\mathrm{HAI}(p=1.0)$. In a series of Cox regression analyses investigating timing to first HAI among those who did and did not develop HAI during ICU stay, the final model included only APACHE II, cumulative invasive device score, and IL-6 rs1800795.

Conclusion: This study provides evidence of a genetic risk for development of HAI. Despite best evidenced based practices some patients will develop HAI. Strict aseptic technique is essential to preventing infection. In addition to eliminating invasive devices as quickly as possible, patients with a high severity of illness may need to be isolated to lower their risk. Early administration of antibiotics not only provides prompt treatment for the initial infection but also lowers risk for subsequent infections.

\section{Document Type}

Dissertation

Degree Name

Doctor of Philosophy (PhD)

Program

Nursing

Research Advisor

Carol L. Thompson, Ph.D. 


\section{Keywords}

Critical Care, Health Care Associated Infections, Polymorphisms, Sepsis, Systemic Inflammation

\section{Subject Categories}

Amino Acids, Peptides, and Proteins | Bacterial Infections and Mycoses | Chemicals and Drugs | Diseases I Medicine and Health Sciences

\section{Comments}

One year embargo expired May 2012 
INFLAMMATORY PROTEINS, GENETIC VARIATION, AND

\title{
ENVIRONMENTAL INFLUENCES ON HEALTH CARE ASSOCIATED
} INFECTION DEVELOPMENT IN SEPSIS

\author{
A Dissertation \\ Presented for \\ The Graduate Studies Council \\ The University of Tennessee \\ Health Science Center
}

\author{
In Partial Fulfullment \\ Of the Requirements for the Degree \\ Doctor of Philosophy \\ From The University of Tennessee
}

By

Reba Antionette Umberger

May 2011 
Copyright (C) 2011 by Reba Antionette Umberger.

All rights reserved. 


\section{DEDICATION}

This dissertation is dedicated in memory of my loving grandmother, Nell Acree (1923-2010). 


\section{ACKNOWLEDGEMENTS}

I would like to first express gratitude to my family and friends for their patience, love, and support throughout this endeavor. They gave me strength and encouragement when I need it. I appreciate their understanding of my time constraints while pursuing this degree and working full time. I am humbled and grateful for the Lord's guidance through the various challenges I have experienced.

I would like express my sincere appreciation to my dissertation chair, mentor, and advisor, Dr. Carol L. Thompson, whose guidance was invaluable. She has been an excellent role model in leadership, and helped me to stay focused while providing feedback and support throughout this process. I would also like to thank my committee members: Dr. Ann Cashion, Dr. David Kuhl, Dr. Jim Wan, and Dr. Ryan Yates. Each contributed significantly to this dissertation project and their guidance and support have been indispensable.

Many have contributed to my development as a nurse researcher and scientist. In addition to my committee, there are several College of Nursing faculty members who have contributed to my education and broadened my perspective. They include Dr. Mona Wicks, Dr. Carolyn Graff, and Dr. Patricia Cowan, who all have served as Program Chairs during my studies. I would like to acknowledge Dr.Veronica Engle, for designing seminar projects that contributed to early stages of this dissertation. I would also like to acknowledge the Office of Research and Grant Support (Dr. Mona Wicks, Ms. Gail Spake, and Ms. Deborah Talley) for review and assistance with grants submitted, as well as assistance with research posters. I am grateful to Ms. Spake for her initial review of this dissertation. Finally, Dr. Thompson and Dr. Cashion encouraged me to attend NIH's Summer Genetics Institute in 2008, which significantly influenced this dissertation.

I would also like to thank my clinical co-investigators at the VAMC, Dr. G. Umberto Meduri and Dr. Muthiah P. Muthiah, who allowed me access to patients in the MICU and provided their medical expertise as needed. My first experience in research, as a research nurse, was given to me by Dr. Meduri and he inspired me to further my education as a researcher and pursue doctoral education. Work from his laboratory by Dr. Siva Kanagat generated ideas for this dissertation project. I would like to thank Dr. Andy Bell for his assistance in identifying potential subjects and in reviewing de-identified data with me for appropriate antibiotic usage. I also appreciate the MICU nurses who notified me of new patients and witnessed informed consents. I am grateful to Mr. Peter St. Arnold, Mrs. Fredrika Curry, and Mr. Leighton Legos for their regulatory guidance at the VAMC IRB. I would also like to thank Mr. Barry Powell for providing me a place to work in the VAMC library and access to a photocopier for de-identified records.

Several individuals have contributed to laboratory components of this study. Dr. Ted Strom allowed my use of the VAMC Core Laboratory for specimen processing and temporarily storage, and the Core Laboratory staff were helpful in providing access. I would like to express gratitude to Dr. Ryan Yates for allowing me to use space in his 
laboratory to complete cytokine analysis and DNA isolation, and for providing guidance. His doctoral student, now Dr. Karin Emmons, was an essential resource by provided me with training and support in the use of Luminex for cytokine measurement. Additionally, his laboratory technician, Mrs. Wenbo Ge, provided assistance with equipment as needed. I am also grateful to the Molecular Resource Center faculty and staff, Dr. William Taylor and Mrs. Felecia Waller, for their training and assistance with genotyping.

This project was funded by University of Tennessee Health Science Center's College of Nursing (cytokine kits) and Millipore (additional cytokine kits). A grant from the International Society of Nurses in Genetics provided funding for genetic analysis, and the College of Nursing provided additional funding for expenses not covered by this grant. The College of Graduate Health Sciences provided travel awards for presentation of various aspects of this dissertation. 


\begin{abstract}
The purpose of this study was to determine the impact of baseline systemic inflammation (pro-inflammatory cytokine, anti-inflammatory cytokine, and their ratio), genetic variability, and environment on the development of health care associated infections (HAI) among sepsis patients during their ICU stay (up to 28 days).

Methods: A prospective observation study was conducted at the Veterans Affairs Medical Center in the Medical Intensive Care Unit over an 18 month period. A total of 78 patients were enrolled within 72 hours of presenting to the ICU with sepsis. Patient were excluded if they were receiving immunosuppressants (chemotherapy or greater than one $\mathrm{mg} / \mathrm{kg}$ of prednisone or equivalent dose), immunosuppressed (AIDS, cancer), or had liver failure (Child Pugh category $\mathrm{C}$ or higher). Baseline plasma and buccal swabs were collected. Patients were followed prospectively through their ICU stay (or for a maximum of 28 days) for the development of HAI as defined by CDC guidelines. Primary variables included baseline IL-6 and IL-10 levels, IL-6 SNP rs1800795, IL-10 SNP rs1800896, APACHE II, invasive devices, and development of HAI.
\end{abstract}

Results: A total of 17 HAI were identified with $64 \%$ caused by Candida. There were no significant differences in levels of pro-inflammatory cytokine, anti-inflammatory cytokine, or their ratio among subjects who did and did not develop at least one HAI during their ICU stay. There were also no significant differences in rs 1800795 or rs1800896 genotypes for those who did and did not develop HAI; however, racial differences were detected in genotypes among white and black patients with sepsis who did and did not develop HAI. There was a significant difference in rs1800795 genotype among black patients with sepsis who did not develop HAI compared to whites patients with sepsis who did not develop HAI $(\mathrm{p}=0.006)$. Specifically, black patients had a lower CG (17.4\% vs. $42.1 \%)$ and higher GG (82.6\% vs. $42.1 \%)$ than white patients. There were no racial differences when comparing white and black sepsis patients who developed HAI $(p=1.0)$. In a series of Cox regression analyses investigating timing to first HAI among those who did and did not develop HAI during ICU stay, the final model included only APACHE II, cumulative invasive device score, and IL-6 rs1800795.

Conclusion: This study provides evidence of a genetic risk for development of HAI. Despite best evidenced based practices some patients will develop HAI. Strict aseptic technique is essential to preventing infection. In addition to eliminating invasive devices as quickly as possible, patients with a high severity of illness may need to be isolated to lower their risk. Early administration of antibiotics not only provides prompt treatment for the initial infection but also lowers risk for subsequent infections. 


\section{TABLE OF CONTENTS}

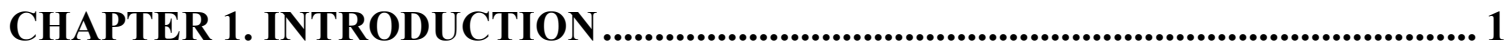

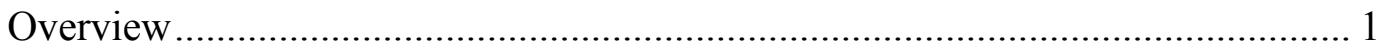

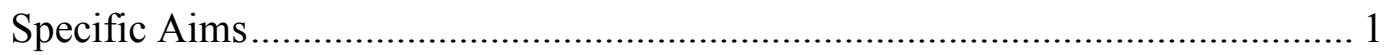

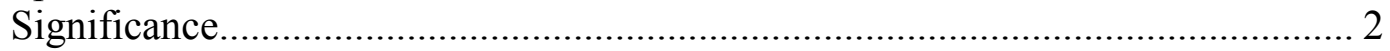

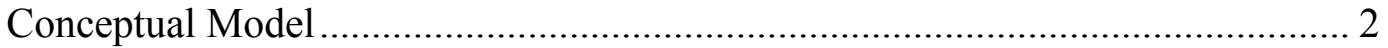

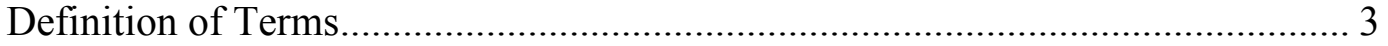

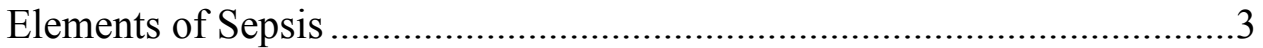

Elements of Severity of Illness ........................................................... 5

Elements of Predisposing Factors .........................................................5

Elements of Inflammatory Response ...................................................6

Health Care Associated Infections ........................................................6

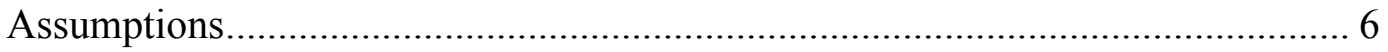

Potential Limitations .............................................................................. 7

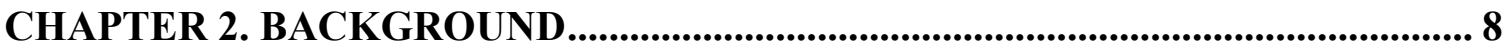

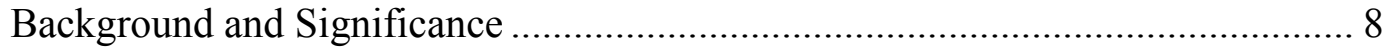

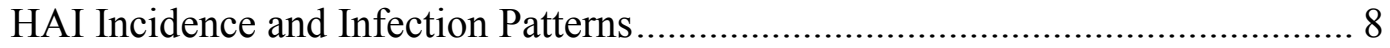

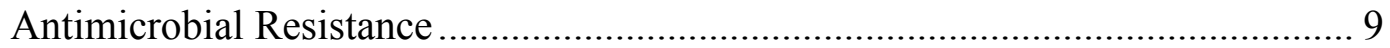

Risk Factors for Developing HAI .............................................................. 9

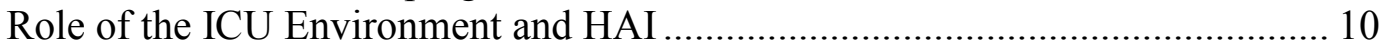

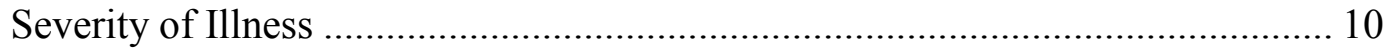

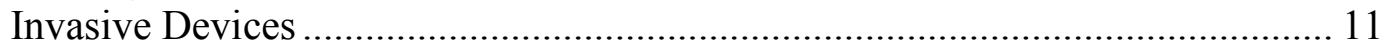

Systemic Inflammatory Response Syndrome ............................................. 11

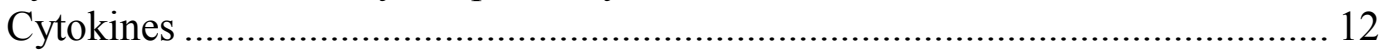

HAI Risk Posed by the Inflammatory Response ......................................... 12

Rationale for Selecting IL-6 and IL-10 ..................................................... 15

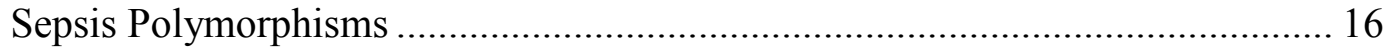

Cytokine Gene SNPs for IL-6 and IL-10 ...................................................... 16

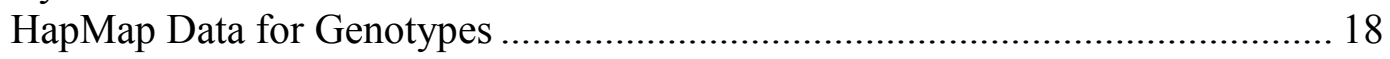

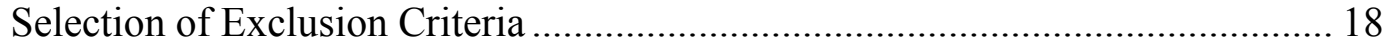

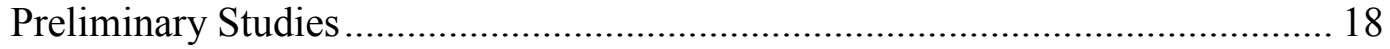

CHAPTER 3. METHODS.................................................................................................. 23

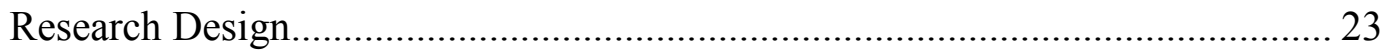

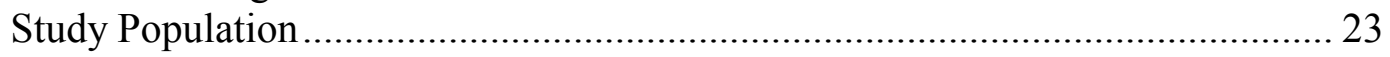

Inclusion Criteria ..........................................................................23

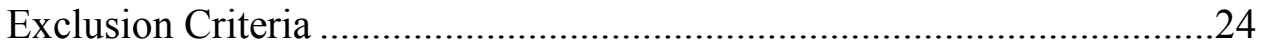

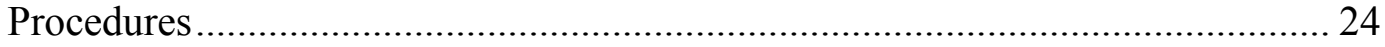

Summary of Procedures ..................................................................24

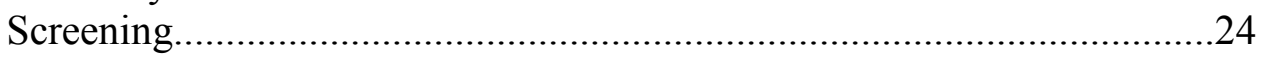

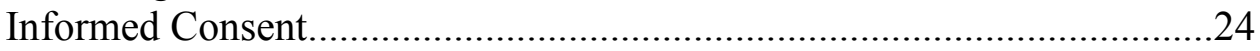




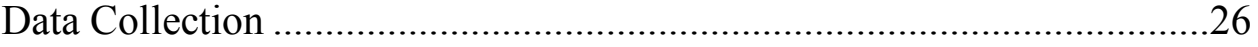

Reliability and Validity of Common ICU Measures .................................26

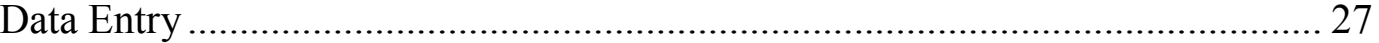

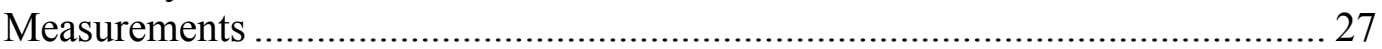

Health Care Associated Infections........................................................27

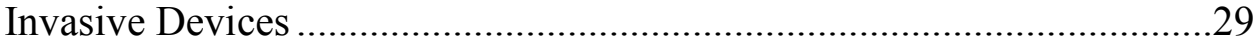

Specimen Processing and Analysis................................................................ 29

Plasma Processing............................................................................

Cytokine Analysis ..................................................................................

Reliability and Validity of Cytokine Measures .........................................31

Buccal Swab Collection for DNA..........................................................32

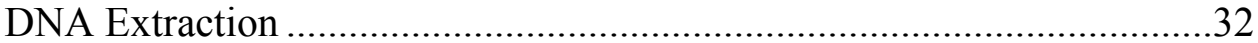

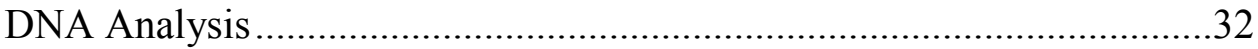

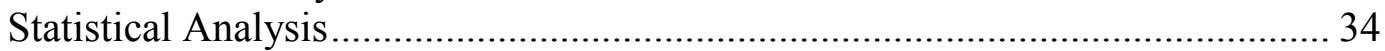

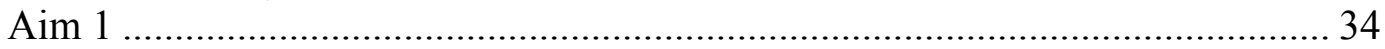

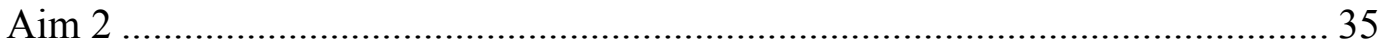

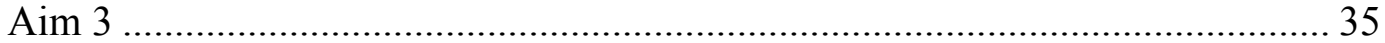

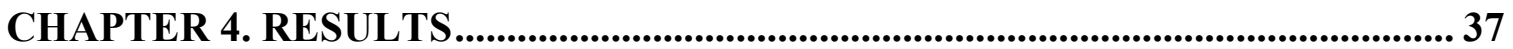

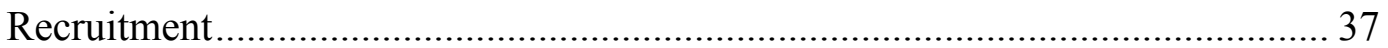

Demographics and Baseline Infections ............................................................ 37

Description of First Health Care Associated Infection ......................................... 45

Differences in Variables among Those Who Did and Did Not Develop HAI...... 45

Differences in Variables by Pro- and Anti-inflammatory Cytokine Quartiles among Those Who Did and Did Not Develop HAI................................................ 49

Cytokine and Genotype Measurements ............................................................. 49

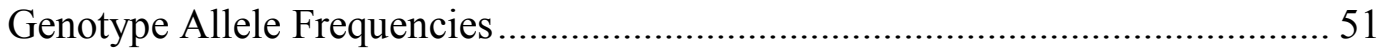

Genotype Comparisons to HapMap 3 Reference Population ................................ 54

Baseline Cytokine Levels by Genotype and Haplotypes ......................................... 54

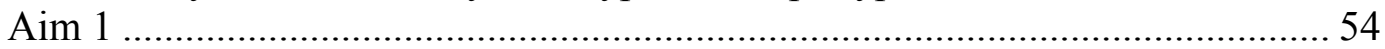

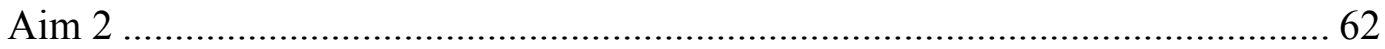

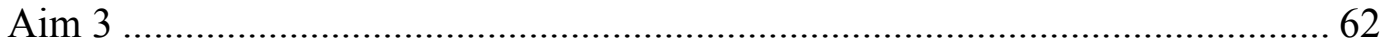

CHAPTER 5. DISCUSSION .......................................................................................... 70

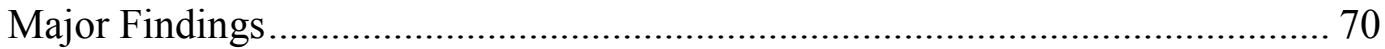

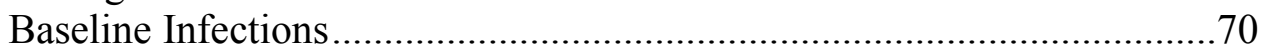

First Health Care Associate Infection ......................................................71

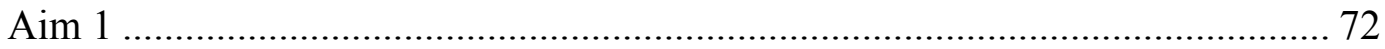

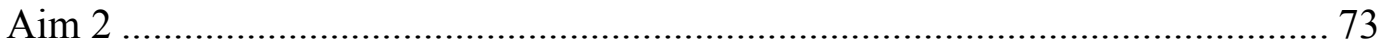

Genotye and Cytokine Level ................................................................73

Genotype and Development of HAI …………………….......................74

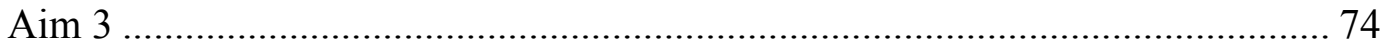

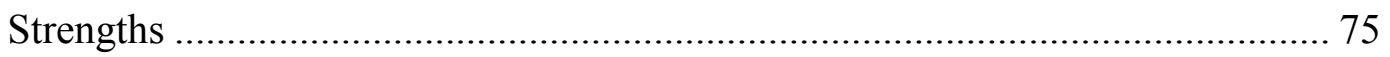

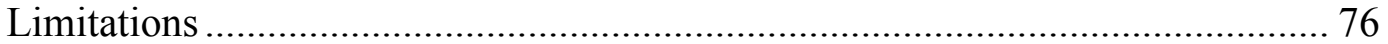

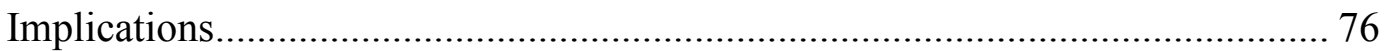


Conclusions.

LIST OF REFERENCES........................................................................................... 78

APPENDIX A. VETERAN'S ADMINISTRATION MEDICAL CENTER INSTITUTIONAL REVIEW BOARD INITIAL

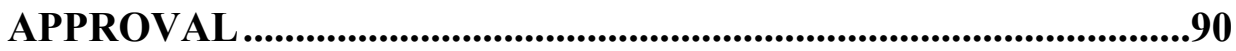

APPENDIX B. VETERAN'S ADMINISTRATION RESEARCH AND

DEVELOPMENT COMMITTEE ANNUAL REVIEW .....................92

APPENDIX C. THE UNIVERSITY OF TENNESSEE HEALTH SCIENCE CENTER INSTITUTIONAL REVIEW BOARD INITIAL

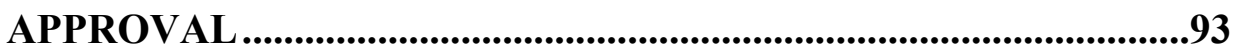

APPENDIX D. VETERAN'S ADMINISTRATION RESEARCH

CONSENT FORM...................................................................................95

APPENDIX E. DATA COLLECTION FORMS ...........................................................101

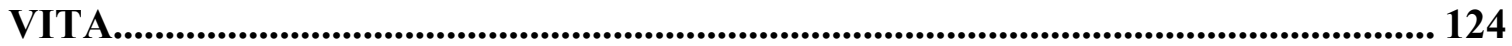




\section{LIST OF TABLES}

Table 2-1. Summary of Cytokine Function..........................................................13

Table 2-2. Selected Cytokine Polymorphism Investigated in Sepsis..........................17

Table 2-3. HapMap 3 RS1800795 Genotype and Allele Frequencies.........................20

Table 2-4. HapMap 3 RS1900896 Genotype and Allele Frequencies........................21

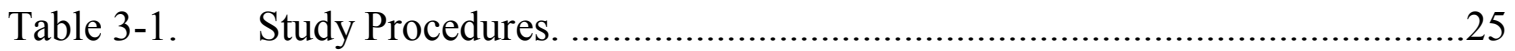

Table 3-2. SNP Primer and Reporter Details for Genotyping.......................................33

Table 4-1. Demographic and Clinical Characteristics................................................40

Table 4-2. Baseline Infection Findings at ICU Admission..........................................42

Table 4-3. Most Common Baseline Micro-organisms. ………......................................44

Table 4-4. Description of First Health Care Associated Infection................................46

Table 4-5. First HAI Micro-organisms (Ranked).....................................................47

Table 4-6. Differences in Variables among Those Who Did and Did Not

Develop Health Care Associated Infections. ...............................................48

Table 4-7. Differences in Variables Pro- and Anti-inflammatory Cytokine

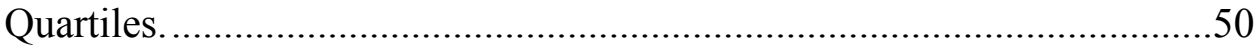

Table 4-8. RS1800795 Genotype and Allele Frequencies...........................................52

Table 4-9. RS1900896 Genotype and Allele Frequencies.........................................53

Table 4-10. Genotype Comparisons of IL-6 and IL-10 SNPs among Sepsis and HapMap Reference Population..................................................................55

Table 4-11. Range of Plasma IL6 and IL10 Levels. ……............................................56

Table 4-12. Median and Inter-quartile Range of Pro- and Anti-Inflammatory Cytokine Levels by Genotypes.

Table 4-13. Comparison of Cytokine Levels among Subjects Developing HAI. .........63 
Table 4-14. SNP Genotypes and Haplotypes for All Subjects by HAI Development and Pro- and Anti-inflammatory Cytokine Quartiles. .........64

Table 4-15. Cytokine levels by Genotype.............................................................65

Table 4-16. Univariate Cox Regression Hazard Ratios for Development of

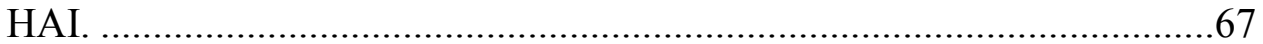

Table 4-17. Final Multivariate Cox Regression Model.............................................68 


\section{LIST OF FIGURES}

Figure 1-1. Development of Health Care Associated Infections in Sepsis.......................4

Figure 2-1. Promoter SNP Locations.......................................................................19

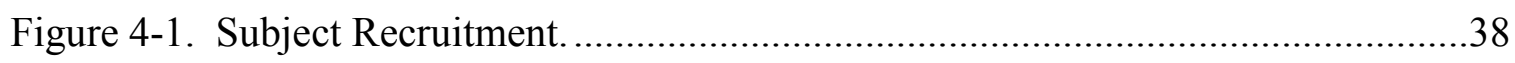

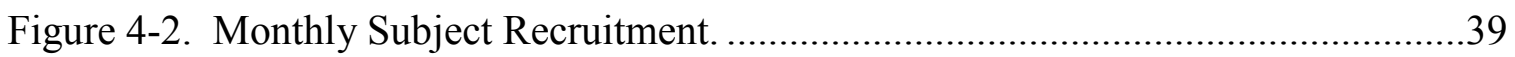

Figure 4-3. Box Plot of Plasma IL-6 levels by IL-6 Genotypes Pre and Post Log

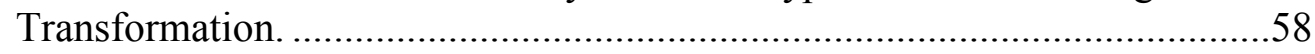

Figure 4-4. Box Plot of Plasma IL-10 levels by IL-10 Genotypes Pre and Post Log

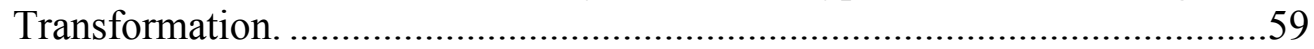

Figure 4-5. Box Plot of Plasma IL-6:IL10 ratios by IL-16 Genotypes Pre and Post Log Transformation.........................................................................60

Figure 4-6. Box Plot of Plasma IL-6:IL10 ratios by IL-10 Genotypes Pre and Post Log Transformation..........................................................................61

Figure 4-7. Log Plasma Cytokine Levels by Genotype............................................66 


\section{LIST OF ABBREVIATIONS}

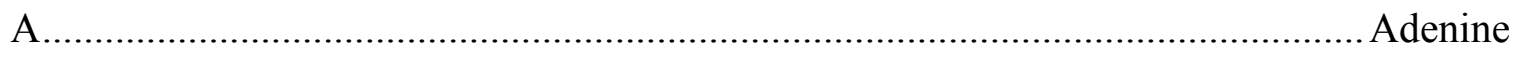

APACHE Acute Physiologic and Chronic Health Evaluation

BAL Bronchoscopic Alveolar Lavage

BMI Body Mass Index

C. Cytosine

CAP Community Acquired Pneumonia

CRP C-Reactive Protein

DNA Deoxyribonucleic Acid

G. Guanine

HAI Health Care Associated Infections HCAP Health Care Acquired Pneumonia IDSA International Disease Society of America IL-1 $\beta$ Interleukin 1 beta IL-1ra Interleukin 1 receptor antagonist

IL-10 Interleukin 10

IL-6 Interleukin 6

LAR ..Legally Authorized Representative MIF Median Florescent Intensity MODS Multiple Organ Dysfunction Syndrome MRC Molecular Resource Center

NCBI National Center for Biotechnology Information NNIS National Nosocomial Infections Surveillance 
PCR Polymerase Chain Reaction

PIRO Predisposition Infection Response Organ Dysfunction SIRS Systemic Inflammatory Response Syndrome

SNP Single Nucleotide Polymorphism SOFA Sequential Organ Dysfunction Syndrome

$\mathrm{T}$ Thymine

TLR Toll-Like Receptor

TNF- $\alpha$ Tumor Necrosis Factor alpha

UTI Urinary Tract Infection

VAP Ventilator Associated Pneumonia

WBC White Blood Cells 


\section{CHAPTER 1. INTRODUCTION}

\section{Overview}

Health care associated infection (HAI) among patients in the intensive care unit (ICU) has been identified as an independent risk factor for hospital mortality. ${ }^{1}$ Patients presenting to the ICU are at high risk for development of $\mathrm{HAI}^{2}$ which can result in prolonged ICU stay, as well as sequelae leading to organ dysfunction and death. ${ }^{2-5}$ The mechanism for development of all HAI is unclear; although, predisposing factors such as invasive devices ${ }^{6-8}$ may be responsible for approximately half of all $\mathrm{HAI}^{9}$ and severity of illness is a predisposing factor. ${ }^{9,10}$ Although the central role of the inflammatory response is to control infections, an exaggerated response may also play a role in the development of HAI.

Cytokines are proteins that orchestrate the inflammatory response. The inflammatory response begins locally and may become systemic. There is usually a balanced inflammatory response, with pro-inflammatory cytokines (i.e., IL-1 $\beta$, TNF- $\alpha$, and IL-6) initiating the inflammatory process against infection, and anti-inflammatory cytokines (i.e., IL-1 ra and IL-10) functioning to down regulate the inflammatory response. ${ }^{11}$ Systemic inflammation is usually manifested with fever or hypothermia, tachycardia, hyperpnea, and leukocytosis or leukopenia.

Baseline severity of illness (measured by APACHE II) correlates with baseline inflammatory response and this process is independent of the causative organism. ${ }^{12}$ The relationship between the development of new HAI and the degree of baseline systemic inflammation or severity of illness has not been fully explored in patients with sepsis. Excessive inflammation has harmful effects and may be a contributing cause of HAI in the ICU.

Sepsis is a complex disease involving a large number of genes. Several polymorphisms have been well characterized in sepsis. ${ }^{13-19}$ Knowledge is accumulating regarding the genetic susceptibility to infectious disease; however, gene-gene, geneenvironment, and host-pathogen interactions should also be considered. ${ }^{20-22}$ Identification of promoter polymorphisms is important when examining an exaggerated inflammatory response. There are a number of important candidate polymorphisms that may be involved in the development of HAI.

\section{Specific Aims}

The purpose of this study is to determine the impact of baseline systemic inflammation (pro-inflammatory cytokine, anti-inflammatory cytokines, and their ratio), genetic variability, and environment on the development of HAI among patients with sepsis during their ICU stay. One of the primary goals of this study is to determine whether exaggerated baseline systemic inflammation increases risk for development of 
HAI during ICU stay. If true, this recognition may promote earlier detection and treatment of infections. Another goal of this study is to identify candidate genes involved in susceptibility of recurrent infections (new HAIs) in sepsis. It is unknown if these genes may differ from genes responsible for the sentinel sepsis event.

The specific aims were to:

1. Investigate whether baseline protein expression levels of pro-inflammatory cytokines, anti-inflammatory cytokines, or their ratios influence the development of subsequent $\mathrm{HAI}$ in patients with sepsis.

2. Investigate the variance in cytokine genes to determine if they influence levels of protein expression or development of HAI.

3. Investigate the effects of protein expression levels, genetic variation, and environment on development of HAI.

\section{Significance}

The findings of this study may provide important new insights into risk factors that contribute to the development of HAI in patients presenting to the ICU with sepsis. It is possible that these findings may be relevant for all patients who develop HAI, as patients may develop sepsis as the result of developing HAI. Targeting early exaggerated inflammation and increased severity of illness may allow earlier detection of HAIs, and promote earlier diagnosis and treatment, perhaps reducing the cost of care (by reducing hospital length of stay) and sequelae that lead to organ dysfunction and death. Thus, these findings may impact nursing and other critical care clinician practice first by helping to identify patients at risk, then implementing stricter targeted infection control practices in efforts to prevent development of HAIs (in addition to current standard and recommended practices), and lastly in early recognition and treatment when HAI occur.

\section{Conceptual Model}

The effects of sepsis and severe sepsis are far-reaching, with severe sepsis affecting approximately 751,000 annually in the US, with a $29-38 \%$ mortality rate. ${ }^{23}$ It is unclear how many of these cases represent new HAI (sepsis occurring at least three days after hospital admission) versus those who were admitted to the hospital or ICU with sepsis. Patients admitted with sepsis seem to be at higher risk for development of HAI. ${ }^{3,4,24}$ An appropriate immune response to an infectious insult ${ }^{25}$ as well as early intervention of appropriate antibiotics, fluid resuscitation, and supportive care ${ }^{26-28}$ are key to a favorable outcome in sepsis. Sepsis is the clinical manifestations of an infectious insult and was defined by Consensus Conference in 1992. ${ }^{29}$ These definitions are still commonly used by clinicians today, and their usefulness was reaffirmed by international 
experts attending the 2001 International Sepsis Definitions Conference, who also proposed a new staging classification system using the acrostic $\mathrm{PIRO}(\mathrm{P}=$ predisposition, $\mathrm{I}=$ insult infection, $\mathrm{R}=$ response, $\mathrm{O}=$ organ dysfunction), similar to the $\mathrm{TMN}$ approach used for tumor staging, to better characterize sepsis. ${ }^{30-34}$ Use of the PIRO model has been described as an effective classification system for researchers given the diversity and heterogeneity of patients with sepsis. ${ }^{35}$ Aspects of this system were incorporated into the conceptual model.

The conceptual model for the development of HAI in sepsis (Figure 1-1) is very complex; yet, this map represents a simplified depiction and incorporates the work of others. $4,31-33,35$ The infectious insult, predisposing factors, severity of illness, and inflammatory response are all key factors to understanding the development of HAIs.

\section{Definition of Terms}

The following terms and definitions are provided for the major concepts in the model. These were the operational definitions used for this study.

\section{Elements of Sepsis}

- Systemic Inflammatory Response Syndrome (SIRS): The presence of at least two of the following: temperature $>38^{\circ} \mathrm{C}$ or $<36^{\circ} \mathrm{C}$, minimum heart rate ( $>$ 90 per minute), elevated respiratory rate $>20$ per minute) or a $\mathrm{PaCO}_{2}$ of $<32$ $\mathrm{mmHg}$, and an abnormal white blood cell count $(>12,000$ or $<4,000$ cells $\left.\mu \mathrm{L}^{-1}\right)$ or bandemia $(>10 \%){ }^{29}$

- Sepsis: SIRS with an identified or suspected source of infection. ${ }^{29}$

- Severe Sepsis: The manifestation of sepsis with organ dysfunction. ${ }^{29}$

- Sequential Organ Dysfunction Assessment (SOFA): The SOFA was used to assess the degree of organ failure among participants. The SOFA score include assessment of six organs (Respiratory, Coagulation, Liver, Cardiovascular, Central nervous system, and Renal). The score for each organ ranges from 0 to 4 , with a total SOFA score ranging from 0 to $24 .^{36}$

- Multiple Organ Dysfunction Score (MODS): The MODS score is an alternative approach for assessing these 6 organs assessed by SOFA. ${ }^{37}$ For this study the MODS is used to measure of the number of organs with clinically significant organ dysfunction (range 0 to 6 ). Similar to the SOFA score, the MODS score also provides a range of categories based on organ function from normal to extreme dysfunction. The cutoff for moderate dysfunction is considered to be clinically significant organ dysfunction. One point is assigned for each clinically significant organ dysfunction using the 


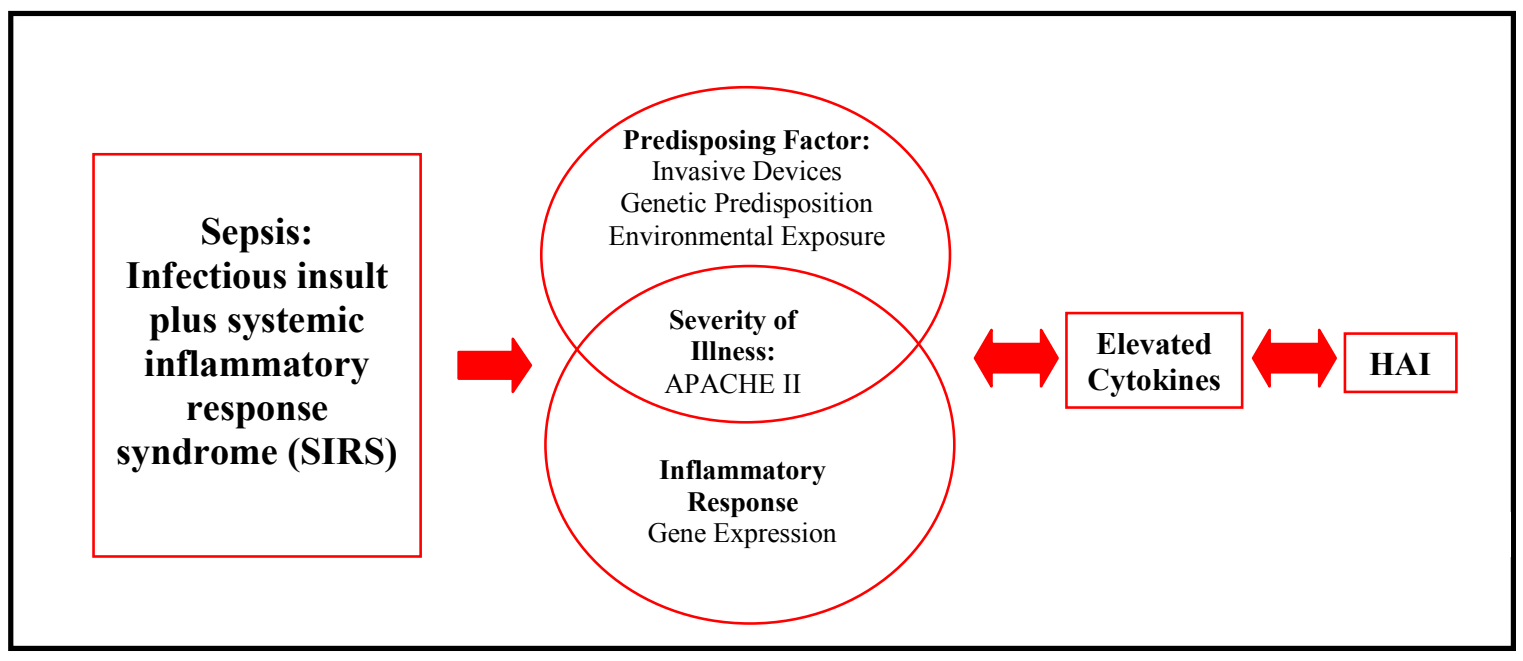

Figure 1-1. Development of Health Care Associated Infections in Sepsis.

Note: This conceptual model incorporates the work of others. ${ }^{4,31-33,35}$ 
following criteria for each organ: (A) Cardiovascular failure is defined by systolic blood pressure less than or equal to $90 \mathrm{mmHg}$ which is not responsive to fluids; (B) Pulmonary failure is defined by a $\mathrm{PaO}_{2}: \mathrm{FiO}_{2}$ of $300 \mathrm{mmHg}$ or less; (C) Central nervous system failure is defined as a Glascow Coma Score of 12 or less; (D) Coagulation failure is defined as a platelet count of 80,000 or less; (E) Renal failure is defined as a creatinine of $2 \mathrm{mg} / \mathrm{dl}$ or less; and (F) Hepatic failure is defined as a total bilirubin of $2 \mathrm{mg} / \mathrm{dl}$ or less. ${ }^{37}$ to fluids; (B) Pulmonary failure is defined by a $\mathrm{PaO}_{2}: \mathrm{FiO}_{2}$ of $300 \mathrm{mmHg}$ or less; (C) Central nervous system failure is defined as a Glascow Coma Score of 12 or less; (D) Coagulation failure is defined as a platelet count of 80,000 or less;

(E) Renal failure is defined as a creatinine of $2 \mathrm{mg} / \mathrm{dl}$ or less; and (F) Hepatic failure is defined as a total bilirubin of $2 \mathrm{mg} / \mathrm{dl}$ or less. ${ }^{37}$

- Infectious insult: Any definitive or suspected infection present at ICU admission will be described as a baseline infection. Suspected infections were defined clinically by the healthcare team.

\section{Elements of Severity of Illness}

- Severity of illness: An objective measure of each participant's illness.

- Acute Physiology and Chronic Health Evaluation II (APACHE II): A commonly used severity of illness classification system designed to capture the worst of 12 physiological variables within the first 24 hours of ICU admission. APACHE II scores range from 0 to 71 with a higher scores associated with a worse outcome. ${ }^{38}$ APACHE II will be used as a measure of severity of illness in this study.

\section{Elements of Predisposing Factors}

- Predisposing factors: Potential risk factors for development of HAI.

- Invasive Devise: Any artificial device that bypasses the body's first line of defense, the integument, is considered an invasive devise. Common invasive devices in this study include endotracheal tubes, tracheostomy tubes, central venous catheters, peripheral venous catheters, arterial catheters, chest tubes, surgical drains, nasal and oral feeding tubes, percutaneous endoscopic gastrostomy tubes, and Foley catheters.

- Genetic Predisposition: Increased susceptibility to a disease due to the presence of one or more gene mutations, and/or a combination of alleles (haplotype), that are associated with an increased risk for the disease. ${ }^{39}$ In this study, single nucleotide polymorphisms (SNPs) will be used to detect genetic variation among participants. 
- Single nucleotide polymorphisms (SNPs): A difference in a single DNA nucleotide which can be measured and may be associated with disease. ${ }^{39}$

- Environmental Exposure: Any potential exposure in a patient's environment that could introduce an infection. In this study, environmental exposure includes invasive devices, nurse-patient ratios $>2: 1$, and administration of blood products. Universal precautions are expected to be followed on all subjects and this includes appropriate hand-washing, protective equipment use, and aseptic technique to prevent cross-infections.

\section{Elements of Inflammatory Response}

- Gene Expression: The process of translating genes into a functional protein product. For the purpose of this study, gene expression will be limited to measurement of cytokine proteins by multiplex bead based assays.

- Cytokines: Protein mediators of the inflammatory response.

\section{Health Care Associated Infections}

- Health care associated infection refers to any infection that occurs after at least three days of hospitalization which is not a recurrence of the baseline infection.

- Specific HAIs are defined by CDC guidelines as described in the methods section. The operational definition for this study will include NEW HAIs that occur after at least three days of admission to the ICU which are not a recurrence of the baseline ICU infection.

- HAI that occur up to 48 hours after ICU admission are considered to be ICU related HAI.

\section{Assumptions}

The following assumptions were made for the purpose of the study:

1. An underlying assumption of this study is that baseline systemic inflammation will be prolonged.

2. HAI will be detected when they occur.

3. Subjects will be classified correctly in analyses based on degree of baseline systemic inflammation and development of HAI. 


\section{Potential Limitations}

This study included the following potential limitations:

1. The usage of corticosteroid therapy may impact the degree and duration of systemic inflammation; thus, potentially limiting the possible impact of systemic inflammation on the development of HAI in participants receiving corticosteroids.

2. The use of corticosteroids may limit fever among participants experiencing HAI, and may result in failure to detect HAI when they occur. It is recommended clinical practice in our ICU to use sepsis surveillance, and thus a high degree of suspicion when steroids are used.

3. There may be predisposing factors for development of HAI that were not measured.

4. Participants receiving mechanical ventilation may have lower DNA yields from buccal swabs due to the presence of a large obtrusive bite block which limited access to swab the inside of the entire cheek as recommended.

5. The investigator is a novice bench researcher, and although efforts were made to accurately follow protocols, it is possible that errors could have influenced results.

6. Endpoint genotyping of the single nucleotide polymorphisms RS1800896 required manual calls in seven samples.

7. Interleukin 6 was selected as a proinflammatory cytokine; however, it does have some anti-inflammatory properties. 


\section{CHAPTER 2. BACKGROUND}

\section{Background and Significance}

Health care associated infections (HAI) affect more than 2 million persons annually in the US, with approximately 35\% occurring in the ICU. A 1999 Institute of Medicine report attributed 44,000-98,000 annual deaths and the associated cost was as high as $\$ 29$ billion. $^{40}$ HAIs (or hospital acquired infections) are typically referred to as infections that are not present or incubating at the time of hospital admission. Infections are "considered to be hospital-acquired if they develop at least 48 [hours] after hospital admission without proven prior incubation." bloodstream infection, pneumonia, and urinary tract infections. Despite standard practices to prevent them, HAIs remain a significant public health care concern.

\section{HAI Incidence and Infection Patterns}

Rates of infections vary but the most common hospital infections in descending order are urinary tract infection, surgical wound infection, lower respiratory tract infection, bacteremia, and others. ${ }^{41}$ Infections are generally classified as primary or secondary and the source may be endogenous or exogenous. ${ }^{41}$ The National Nosocomial Infections Surveillance (NNIS) System collects surveillance information across medical intensive care units in the United States. An analysis of NNIS data $(n=181,993)$ determined the most frequent types of HAI in the ICU were urinary tract infections (31\%), pneumonia (27\%), and primary bloodstream infection (19\%) from surveillance data between 1992 and 1997. ${ }^{42}$ The most common pathogens reported were Coagulatenegative Staphylococcus, Staphylococcus aureus, and Enterococcus spp. The authors provide a comprehensive summary with percentages for each type of infection.

In contrast to the pattern of infections among all US ICU patients, a one day ICU prevalence study conducted in 1992 provides a similar pattern of infection types occurring in European ICU patients. In descending order, Vincent et al. reported the most common ICU infection as pneumonia (46.9\%), lower respiratory tract infection $(17.8 \%)$, UTI (17.6\%), and bacteremia (12\%) in a large European study of 10,038 subjects in 1,417 intensive care units with a total of 4051 infections. ${ }^{43}$ These authors reported the most common organism as Enterobacteriaceae (34.4\%) followed by Staphylococcus aureus (30.1\%), Pseudomonas aeruginosa (28.7\%), Coagulate-negative Staphylococci (19.1\%), and fungi (17.1\%). Organisms were not reported for each type of infection separately. $^{43}$

In a more recent study of a combined medical and surgical ICU, the most frequent infections (in descending order) were ventilator associated pneumonia, surgical site infection, lower respiratory tract infection, intrabdominal infection, sinusitis, soft tissue or skin infection, bacteremia, and finally, UTI. ${ }^{2}$ This study examined risk factors for mortality and organisms were not provided. The pattern of infections in the ICU varies. 
The rates of NI may vary widely depending on the type of ICU and population served. Eggiman and Pitet provide a comprehensive list of infection rates by type of ICU. ${ }^{40}$ The causative organism may not be detected in all infections and the rate of viral infections in the ICU may be underappreciated. There is evidence that despite causative organism type (gram negative, gram positive, polymicrobial, or fungal) the host's inflammatory and coagulation response is similar in severe sepsis. ${ }^{12}$

\section{Antimicrobial Resistance}

Several factors in the ICU contribute to promoting antimicrobial resistance. These include (1) cross-transmission often caused by the urgency of care that may result in inconsistent aseptic technique or hand washing, (2) compromise of host defenses through the use of invasive devices which may become colonized, (3) the use of antibiotics. ${ }^{44}$ The emergence of antibiotic resistant organisms such as Methicillin resistant Staphylococcus aureus (MRSA), Vancomycin resistant Enterococcus, and other multidrug resistant organisms are major concerns. ${ }^{44-47}$ Caution in antibiotic prescribing in both the community and hospital setting has been advised. Only approximately $30 \%$ of antimicrobials used in hospitals are being given as definitive therapy for known infection associated pathogens based on microbiologic susceptibility. ${ }^{44}$ Cycling empiric antimicrobial therapy has been proposed to reduce antimicrobial resistance and has had favorable results in the susceptibility profile of gram positive but not gram negative organisms. ${ }^{48,49}$

The concept of antibiotic cycling to reduce antimicrobial resistance has been around since at least $1986 .{ }^{50}$ Since microorganisms are generally adaptive to their environment, limiting the introduction of particular antimicrobial for periods of time may help to decrease resistance patterns. There are few controlled trials published. A number of methods have been proposed to assist with cycling including the use of hospital formularies. ${ }^{51}$ The use of local antibiograms should be used to guide empiric therapy and antimicrobial therapy should be guided by susceptibility testing as soon possible since a delay in appropriate antimicrobial therapy worsens outcome. Antibiograms are frequently used to adjust antibiotics in the ICU; however, failure to use them in other settings is a contributor to antimicrobial resistance. ${ }^{52}$

\section{Risk Factors for Developing HAI}

Patients admitted to the ICU have an increased risk for developing HAI which may be related to underlying disease conditions, impaired immunity, invasive devices, inappropriate aseptic technique, or secondary infections after broad spectrum antibiotics. ${ }^{2}$ Eggimann identifies severity of illness, prolonged length of stay, and vascular access as independent risk factors for the development of HAI. He provides a thorough review of infection control practices in the ICU, and attributes most infections to inadequate infection control practice, and also implicates understaffing and overcrowding of the ICU as contributing factors. ${ }^{40}$ Genetic susceptibilities are known to increase the risk of 
developing infections; ${ }^{20,21}$ however, the influence of these genetic susceptibilities on development of subsequent HAI is unknown. Recommendations for standard practices to prevent the development of HAIs in the ICU have been reported by the Infectious Disease Society of America (IDSA), the Center for Disease Control (CDC), and others. ${ }^{53-57}$

\section{Role of the ICU Environment and HAI}

As noted in the prior sections, being a patient in the ICU presents several inherent risks factors for the development of HAI including: presence of invasive devices, use of broad spectrum antibiotics, use of blood products, and generally more physical contact with the health care team administering care. A standard patient to nurse ratio for most critical care units is 2:1 depending upon patient acuity. The implementation of open visitation in the ICU has the potential to introduce additional risk of cross-contamination to this vulnerable population. Appropriate hand hygiene is essential to prevent crosscontamination, in addition the CDC reports the potential role of HAI transmission by health care provides wearing rings and long or artificial nails. ${ }^{58}$ Routine isolation practices are used when any multi-drug resistant organism is detected. ${ }^{59}$ The floors are mopped and cleaned daily as well as between patients according to CDC guidelines. ${ }^{60}$ The areas closest to the patient including the bed side rails, bed controls, bedside tray, and call light are typically only cleaned between patients unless soiled and may represent items that needs to be targeted for more frequent cleaning.

\section{Severity of Illness}

Severity of illness can be quantified by using the Acute Physiology and Chronic Health Evaluation (APACHE) II, a composite score that ranks a patient's worst physiologic functioning within the first 24 hours of ICU admission. ${ }^{38}$ The APACHE II is in the public domain and was used for this study. Severity of illness has been investigated as a risk factor for developing HAI. Girou et al, conducted a retrospective case-control study $(\mathrm{n}=82)$ with 1:1 matching based on initial severity of illness using APACHE II and also obtained serial APACHE II scores to determine the influence of severity of illness on development of infections. Among several variables assessed, they identified day three APACHE II score as significantly higher in the cases $(p=0.04) .{ }^{61}$ All patients had similar APACHE II scores at baseline, and worsening of APACHE II score provides evidence of worsening severity of illness among those who developed HAI. In contrast, Vincent et al, in a one day prevalence study across 17 European ICUs $(n=10,038)$, identified a high APACHE II score of greater than 31 to be independently associated with risk of mortality; however, they reported the highest HAI rates in patients with APACHE II scores ranging from $11-20{ }^{43}$ 


\section{Invasive Devices}

Invasive devices bypass the body's first line of defense, the integument, and may lead to infection if penetrating organisms are not promptly sequestered by the innate immune system. ${ }^{25}$ Common invasive devices in the ICU include endotracheal tubes, central venous catheters, peripheral venous catheters, arterial catheters, chest tubes, nasal feeding tubes, and Foley catheters. Several studies have been published describing the relationship between invasive devices and infections. ${ }^{8,40,42,53,62-64}$ In many cases HAIs are used as a benchmark for quality of care, and Maki suggests that peripheral intravenous catheters (previously considered low risk) should begin to be targeted by infection control practices to reduce invasive device related infections. ${ }^{64}$ The use of antibiotic impregnated devices and targeted strategies have helped to reduce the rates of invasive devise related infections. ${ }^{65,66}$

Invasive devices may be colonized with bacteria or fungi through biofilm formation. Biofilms generally forms when microorganisms adhere to invasive devices which provide them with an environment capable of evading antibiotics. They initially adhere to the foreign surface and then begin to secrete extracellular polymeric substance (EPS) to protect formation of the biofilm and attract other organisms. The microorganisms within a biofilm exhibit different physiologic and growth characteristics than do free floating (or planktonic) organisms. ${ }^{67}$ They use quorum sensing to communicate and control the gene expression of other organisms within the biofilm, and to control each phase of biofilm formation: attachment, cell-to-cell aggregation, proliferation, EPS production, grown, and detachment or degradation. ${ }^{68}$ Acute infections are generally caused by the planktonic (free floating micro-organisms) but the role of biofilm formation on chronic and acute infection is an area of research. ${ }^{67,69,70}$

\section{Systemic Inflammatory Response Syndrome}

The body is subject to a variety of insults daily and the immune system is usually able to isolate and clear potential pathogens without systemic effects. When the body is not able to maintain inflammation at the local level, inflammation becomes systemic. The systemic inflammatory response syndrome (SIRS) is the clinical manifestation of the body's host defense response to a variety of insults and initially manifests in at least two of four major organ systems (cardiovascular, hematopoietic, neurologic, and respiratory system). These insults can be infectious or noninfectious in nature. Noninfectious insults include pancreatitis, burns, trauma, tissue ischemia or necrosis, massive transfusion, chemical aspiration, foreign bodies, and immune hypersensitivity reactions. ${ }^{29,71,72}$

The goal of the inflammatory response is to control the initial insult or injury. Three primary responses (vascular, neutrophil, and plasma protein) function to increase blood flow to the affected area, increase vascular permeability to allow leukocytes and plasma proteins into the site of injury or infection. ${ }^{71}$ SIRS is a dysregulated inflammatory response caused by activation of inflammatory cells. It is a generalized response regardless of the type of insult. This dysregulated inflammatory response leads 
to generalized systemic inflammation, damaged vascular endothelium, altered immune function, fluid shifts, and organ hypoperfusion. ${ }^{71}$ If this process is not controlled, it can progress to multiple organ dysfunction syndrome. ${ }^{71}$ SIRS occurs in approximately one third of all hospitalized patients and one half of ICU patients. ${ }^{71}$

\section{Cytokines}

Cytokines are produced by a variety of cells (lymphocytes, macrophages, epithelium, endothelium, connective tissue, adopocytes, and myocytes) which modulate the function of other cell types. ${ }^{72}$ Cytokines are pleotropic which means that one cytokine can act on a number of cell types and have many effects. ${ }^{73}$ They have autocrine, paracrine, and endocrine effects. Cytokines play a role in both acute and chronic infections. $^{72}$ Activated lymphocytes and macrophages are their primary secretor. Cytokines are not stored in their active states in the cell. Their production requires new mRNA and protein synthesis. Most are encoded as pro-peptides, transported to the Golgi body for glycosylation, and secretion as smaller mature cytokine proteins. $^{74}$

A growing number of cytokines are still being discovered. Interleukins mediate communication between leukocytes. IL-1 $\beta$ and TNF- $\alpha$ promote the acute inflammatory response, thus they are typically referred to as pro-inflammatory cytokines. IL-6 is another potent inducer of the acute inflammatory response. It stimulates the liver to release acute phase proteins. Although IL-6 is often referred to in the literature as a proinflammatory cytokine, evidence is accumulating regarding its' anti-inflammatory properties. IL- 6 inhibiting effects of IL-1 $\beta$ and TNF- $\alpha{ }^{75}$ IL1-ra and IL-10 are both know as anti-inflammatory cytokines. Table 2-1 summarizes the function of well known pro- and anti-inflammatory cytokines.

\section{HAI Risk Posed by the Inflammatory Response}

There is some evidence that cytokines may enhance extracellar and intracellular growth of bacteria ${ }^{76-79}$ and that anti-inflammatory cytokines may promote the development of infections. ${ }^{11,80}$ Kanangat et al. found, in an in vitro model, that monocytes that were primed with higher levels of proinflammatory cytokines experienced higher levels of intracellular bacterial growth; whereas monocytes primed with lower levels of proinflammatory cytokines experienced lower levels of intracellular bacterial growth. ${ }^{77}$ In another in-vitro study, Kanangat also found that cytokines enhanced the extracellular growth of Staphylococcus aureus. ${ }^{76}$ Because of the complexity of the inflammatory response which includes redundancy of the inflammatory networks, competing feedback loops, and simultaneous amplification pathways ${ }^{35}$, it is uncertain that a relationship that exists in-vitro will exist in-vivo.

It is possible that high levels of cytokines function as bacterial growth factors ${ }^{76-79}$ or that anti-inflammatory cytokines may promote the development of infections. ${ }^{11,80}$ It is also possible that an exaggerated inflammatory response may promote HIAs by inhibition 
Table 2-1. Summary of Cytokine Function.

\begin{tabular}{|c|c|c|}
\hline Cyokine & Function & Reference \\
\hline IL-1 $\beta$ & $\begin{array}{l}\text { Mediate innate immunity. T cell activation. } \\
\text { Macrophage activation. Fever. Induces production of } \\
\text { IL-6. Induce endothelial adhesion molecules. }\end{array}$ & $25,73,81$ \\
\hline TNF- $\alpha$ & $\begin{array}{l}\text { Mediate innate immunity. Local inflammation. } \\
\text { Induces acute inflammation by activation of and } \\
\text { endothelial cells. Induce endothelial adhesion } \\
\text { molecules. Activates macrophages and inducted } \\
\text { nitric oxide production. Fever. Mobilization of } \\
\text { metabolites. Shock. }\end{array}$ & $25,73,81$ \\
\hline IL-6 & $\begin{array}{l}\text { Mediate innate immunity. Acute phase protein } \\
\text { production. Fever. T and B cell growth and } \\
\text { differentiation. Activates T and B cells. Down- } \\
\text { regulates the synthesis of IL-1 and TNF. }\end{array}$ & $25,73,75,82$ \\
\hline IL-10 & $\begin{array}{l}\text { Potent suppressor of macrophage functions by } \\
\text { inhibiting cytokine release. Inhibits } \mathrm{T}_{\mathrm{H}} 1 \text { cells. Effects } \\
\mathrm{B} \text { cells to increase MHC class II. Down regulate the } \\
\text { immune response. Deactivates monocyte/macrophage } \\
\text { proinflammatory cytokine synthesis. }\end{array}$ & $25,73,75$ \\
\hline IL-1ra & $\begin{array}{l}\text { Inhibits IL-1 by binding to the receptor by } \\
\text { competitive inhibition of the receptor IL-1 site. }\end{array}$ & 25,75 \\
\hline
\end{tabular}

Note: IL denotes interleukin. TNF denotes tumor necrosis factor. IL1ra denotes IL-1 receptor antagonist. MHC denotes major histocompatability complex. T cells are $\mathrm{T}$ lymphocytes that were derived from the thymus. B cells are B lymphocytes that were derives from bone marrow. $\mathrm{T}_{\mathrm{H}} 1$ denotes a class of $\mathrm{T}$ helper cells. 
of Toll-like receptors (TLRs) ${ }^{83}$ or by impairment of neutrophil function. ${ }^{84}$ Toll-like receptors are a family of proteins that act as pattern recognition receptors and enable cells to recognize pathogen-associated molecular patterns. TLRs allows the innate immune system to immediately recognize highly conserved bacterial, viral, and fungal components. ${ }^{83,85}$ An evolutionary mechanism that evolved to protect the host from the harm of an overly responsive immune system, such as exaggerated IL-6 levels, is the inhibition of Toll-like receptor signaling by IL-1 receptor associated kinase-M. ${ }^{86}$ High IL-6 levels have also been reported in a small study $(n=21)$ of patients with HAIs who had impairment of neutrophil function resulting in impaired phagocytosis and bacterial killing. ${ }^{84}$ If the relationship of exaggerated or high IL-6 levels and development of HAIs is confirmed in this study, the underlying mechanism will require investigation.

There have been no clinical studies to determine how cytokines influence bacterial growth in sepsis. However, in 1997, Headley et al. studied the effect of infections and the inflammatory response in ICU patients $(n=34)$ with Acute Respiratory Distress Syndrome (ARDS). They found that the outcome in ARDS was not related to the development of HAIs but that it was attributed to the magnitude and duration of the inflammatory response. It is unclear if they specifically tested the association of baseline level of inflammation and development of subsequent HAI. One important finding was that when a new HAI developed, it was not accompanied by an increase in levels of inflammatory proinflammatory cytokines. ${ }^{87}$ The same authors have reported in the past that persistent elevation of inflammatory cytokines was a poor predictor of outcome. The authors provided evidence that non-survivors have high levels of inflammatory cytokines at baseline which persisted for at least the first 10 days, whereas, survivors had a lower baseline level which decreased over the first 10 days. ${ }^{88}$ It is unknown how long this relationship persists since data were not shown beyond 10 days. A similar persistent elevation of the pro-inflammatory cytokine, IL-6, was recently reported in non-survivors with severe sepsis. ${ }^{89}$ The first week to 10 days may describe a more homogenous population of early infections; however, infections may also be described as early, middle, and late infections. ${ }^{90}$ The relationship of persistent systemic inflammatory, when defined clinically as systemic inflammatory response syndrome (SIRS) and not based on cytokine levels, has been shown to significantly increase the risk for developing HAI in a trauma population. ${ }^{90,91}$

The relationship of severity of illness and health care associated infections has been described. ${ }^{10}$ McCluster et al. similarly report that patients who developed HAI had higher APACHE III scores than those who did not develop HAI but the relationship was not statistically significant. ${ }^{9}$ Kinasewitz et al. analyzed multiple biomarkers from the PROWESS data set $(n=1,690)$, a randomized controlled trial of recombinant human activated protein $\mathrm{C}$ in severe patients with sepsis. They found significant correlations in biomarkers relative to increasing levels of APACHE II. Of interest, median and interquartile ranges of IL-6 ( $\mathrm{pg} / \mathrm{ml})$ were increased for all participants with an increase for each quartile of APACHE II ( $1^{\text {st }}$ quartile: 289 (245 - 369), $2^{\text {nd }}$ quartile: 384 (322 $489), 3^{\text {rd }}$ quartile: 623 (494 - 829), $4^{\text {th }}$ quartile: $\left.1043(809-1613), p<0.001\right)$. Their data for IL-10 ( pg/ml) was inconclusive as $59 \%$ were below the detection limit: ( $1^{\text {st }}$ quartile: $\leq 10(10-10), 2^{\text {nd }}$ quartile: $\leq 10(10-10), 3^{\text {rd }}$ quartile: $\leq 10(10-30), 4^{\text {th }}$ quartile: $\leq 10$ 
$(10-27), p=0.001) .{ }^{12}$ The APACHE II ranges for each quartile were: $1^{\text {st }}$ quartile ( 3 $-19), 2^{\text {nd }}$ quartile $(20-24), 3^{\text {rd }}$ quartile $(25-29)$, and $4^{\text {th }}$ quartile $(30-59)$. Criteria for the original study included severe sepsis with at least three signs of systemic inflammation and at least one organ failure. ${ }^{92}$

It is assumed that patients with sepsis who present with a high degree of baseline systemic inflammation will have persistent inflammation which may contribute to the development of HAI. The role of pro- versus anti-inflammatory cytokines in the development of sepsis is an area of debate. Assessment of the cytokine profile may be more predictive than assessing individual cytokines in isolation. ${ }^{93}$ Although other more common mechanisms for the development of HAI exist, such as poor aseptic techniques, the relationship of systemic inflammation and development of HAI in patients with sepsis presents a novel approach which is explored in this dissertation.

\section{Rationale for Selecting IL-6 and IL-10}

When planning this dissertation study, it was to be limited it to one proinflammatory cytokine, one anti-inflammatory cytokine, and one ratio. Before this project began, there was concerned about the use of IL-1 $\beta$ and IL-1ra due to reports of low detection limits in the literature. In addition to measuring IL-1 $\beta$ and IL-1ra, we also measured TNF- $\alpha$, IL-6, and IL-10. IL-6 and IL-10 were included as an alternative pro- to anti- inflammatory ratio since they were less likely to be below detection limits and because there were other reports examining these ratios. ${ }^{94}$ A large number of cytokine values were below the detection limit for IL1B (66 or 84\%) and IL1ra (50 or 64\%) which indeed limited comparison of their ratios. These low detection limits also limited our ability to detect a difference in cytokine levels for the associated SNP. It is probable that these very low concentrations (below the $3.2 \mathrm{pg} / \mathrm{ml}$ threshold used in this study) are biologically important, despite the fact that they cannot be precisely measured. A total of 5 (6.4\%) IL-6 levels and 29 (37.2\%) IL-10 levels were below the detection limit.

Numerous articles throughout the literature include IL-1 $\beta$, TNF- $\alpha$, and IL-6 as pro-inflammatory cytokines; however, it should be noted that IL-6 also has many other functions. IL-6 not only induces the acute phase response causing the liver to release $\mathrm{CRP}$ and other acute phase reactants, it is also involved in immunoglobulin switching necessary for acquired immunity. ${ }^{95,96}$ IL-6 may have a protective effect against development of septic shock; ${ }^{96}$ whereas, minute amounts of TNF- $\alpha$ and IL-1 $\beta$ are potent initiators of septic shock. ${ }^{81}$ IL-6 has been shown to consistently correlate with clinical severity of inflammation, autoimmune, and infectious disease; whereas, this relationship is not always clear with IL-1 and TNF. ${ }^{97}$ IL-6 levels have been used to predict fatal outcome in septic shock whereas this has not been shown with TNF levels. Dinarello concludes that among patients with septic shock, IL-6 levels seems to "represent the net effect of biologically active IL-1 and TNF". 97 


\section{Sepsis Polymorphisms}

Sepsis is a complex disease involving a large number of genes. Genes are composed of deoxyribonucleic acid (DNA) which is a polynucleotide chain made from sugar (2-deoxyribose) linked to phosphate backbone with protruding nucleotide bases. These bases are either purines (Adenine and Guanine) or pyrimidines (Cytosine and Thymine). A polymorphism is a common variation (greater than one percent) in the DNA sequence among individuals; whereas, a mutation has a frequency of less than one percent. A single nucleotide polymorphism is the substitution of one of the four nucleotide bases with another nucleotide base and may confer a survival advantage. ${ }^{98}$ Another type of polymorphism is a tandem repeat or microsatellite polymorphism, in which a number of nucleotides are repeated once or several times. The normal or usual genotype is referred to as the wild type, although wild type can refer to the genotype or phenotype. $^{99}$

Only about 10 percent of the DNA sequence in the human genome codes for genes, and SNPs occur approximately every 1,000 base pairs with most not resulting in a protein or secretion change. ${ }^{98}$ SNPs occurring in the promoter regions of genes have potential to influence the level of gene expression and are likely to be important. Several polymorphisms have been well characterized in sepsis. ${ }^{13-19}$ Knowledge is accumulating regarding the genetic susceptibility to infectious disease; however, gene-gene, geneenvironment, and host-pathogen interactions should also be considered. ${ }^{20-22}$

Identification of promoter polymorphisms is important when examining an exaggerated inflammatory response. There are a number of important candidate polymorphisms that may be involved in the development of HAIs. Table 2-2 includes a brief review of selected polymorphism. Although evidence supports the importance of these polymorphisms in sepsis, a study of young, healthy white males with no smoking or co-morbidity history, found a trend but no clear association between common polymorphisms and cytokine levels in a sepsis model of endotoxin exposure. ${ }^{100}$ There is research indicating that epigenetic factors may down regulation genes as early as 3-5 hours after an infectious insult which could be the reason the investigators did not find an association. ${ }^{101}$ It is reasonable to conclude that patients who have already shown susceptibility to sepsis by presentation to the ICU with sepsis have a different risk profile than these healthy young men. Additionally, polymorphisms in one cytokine gene may enhance expression levels of other cytokine genes. ${ }^{102}$

\section{Cytokine Gene SNPs for IL-6 and IL-10}

The gene for IL-6 is located on chromosome seven on the $\mathrm{p}$ arm. The gene for IL6 consists of 4,856 nucleotides and begins at location 22,766,766. The promoter region lies upstream from the gene. It generally starts approximately $25 \mathrm{bp}$ upstream from the gene starting point but transcription factors have a large "footprint" and their binding to the promoter is necessary prior to the binding of RNA polymerase II and ultimate transcription of IL-6 into mRNA. ${ }^{103}$ There are several SNPs located within the promoter 
Table 2-2. Selected Cytokine Polymorphism Investigated in Sepsis.

\begin{tabular}{|c|c|c|c|c|c|}
\hline Cytokine & $\begin{array}{l}\text { Gene } \\
\text { Location }\end{array}$ & Polymorphism & RS Number & Rationale & Reference \\
\hline IL-1 $\beta$ & $2 q 14$ & $-511 \mathrm{C} / \mathrm{T}$ & rs1143643 & $\begin{array}{l}\text { Mixed results. } \\
\text { Increased risk for } \\
\text { septic shock } \\
(\mathrm{C} / \mathrm{T}) \text {. }\end{array}$ & 104-106 \\
\hline TNF- $\alpha$ & $6 \mathrm{p} 21.3$ & $-308 \mathrm{G} / \mathrm{A}$ & rs 1800629 & $\begin{array}{l}\text { A, increased risk } \\
\text { for sepsis }\end{array}$ & 19,107 \\
\hline IL-6 & $7 \mathrm{p} 21$ & $-174 \mathrm{G} / \mathrm{C}$ & rs 1800795 & $\begin{array}{l}\text { G, higher } \\
\text { cytokine levels, } \\
\text { others lower } \\
\text { levels }\end{array}$ & $16,18,108$ \\
\hline IL-10 & $1 q 31-q 32$ & $-1082 \mathrm{G} / \mathrm{A}$ & rs 1800896 & $\begin{array}{l}\mathrm{G} \text {, shock, more } \\
\text { severe sepsis, } \\
\text { more severe } \\
\text { pneumonia }\end{array}$ & $16,19,107$ \\
\hline IL-1ra & $2 \mathrm{q} 14.2$ & 86-bp repeat & na & $\begin{array}{l}\text { A2, increased } \\
\text { risk for sepsis }\end{array}$ & $16,18,19$ \\
\hline
\end{tabular}

Note: IL denotes Interleukin. TNF denotes tumor necrosis factor. IL1ra denotes IL-1 receptor antagonist. 
region of IL-6. RS1800795, previously known as $-174 \mathrm{G} / \mathrm{C}$, is located at 22,766,645 and has been well described in the literature. It has been associated with both higher and lower levels of IL-6 when position RS 1800795 is encoded by G. ${ }^{16,18,108}$

The gene for IL-10 is located on chromosome one on the $\mathrm{q}$ arm. The gene for IL10 consists of 4891 nucleotides and extends from location 206,945,839 to 206,940,948. The promoter for IL-10 lies upstream from the gene and SNP RS1800896, previously known as $-1082 \mathrm{G} / \mathrm{A}$, is located upstream within the promoter region at 206,946,897. It has been shown that the presence of a $G$ at this position is associated with shock, more severe sepsis, and more severe pneumonia. ${ }^{16,19,107}$

Figure 2-1 shows the location of these SNP upstream from the gene using the National Center for Biotechnology Information's (NCBI) new Sequence Viewer v.2.12.

\section{HapMap Data for Genotypes}

The International HapMap Project began in 2002 with the goal of providing patterns of human DNA sequence variation to enable scientists to investigate genes affecting health, disease, and response to drugs and environmental factors. The HapMap Project is currently in its third phase, HapMap 3, which includes 1,301 samples from eleven human populations, and 270 of those samples originating from prior phases. ${ }^{109}$ HapMap 3 included samples from African ancestry in Southwest USA (ASW) and Utah residents with Northern and Western European ancestry (CEU) were included. Tables 2-3 and 2-4 show the distribution. Note the difference between ASW and Africans from Sub-Sahara. Courtesy: National Human Genome Research Institute.

\section{Selection of Exclusion Criteria}

This study was designed to examine systemic inflammation and its influence on the development of HAI. Patients with a disease process know to decrease immune function and those receiving certain immunosuppressants could confound findings on HAI. This includes patients with cancer and human immunodeficiency syndrome. It also includes patients on high dose corticosteroids or chemotherapy. Acute phase proteins are secreted by the liver in response to inflammation; thus, patients with a liver failure (Child Pugh Score of $\mathrm{C}$ or worse) were also excluded. While the elderly may be more

susceptible to infections due to a functional decline in cell-mediated immunity, ${ }^{110}$ we opted not to exclude patients based on age so that this relationship could be examined if it existed in this population.

\section{Preliminary Studies}

Until recently, there had been no clinical studies examining the relationship of excessive baseline inflammation with the development of subsequent health care 
A.

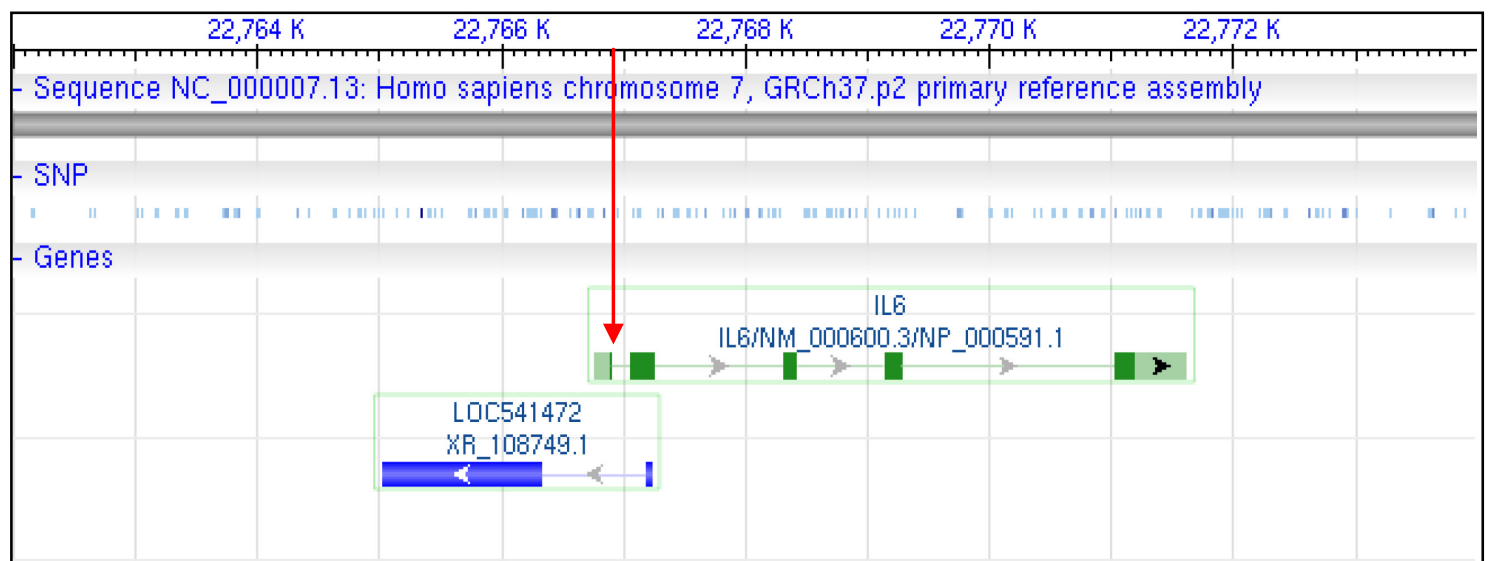

B.

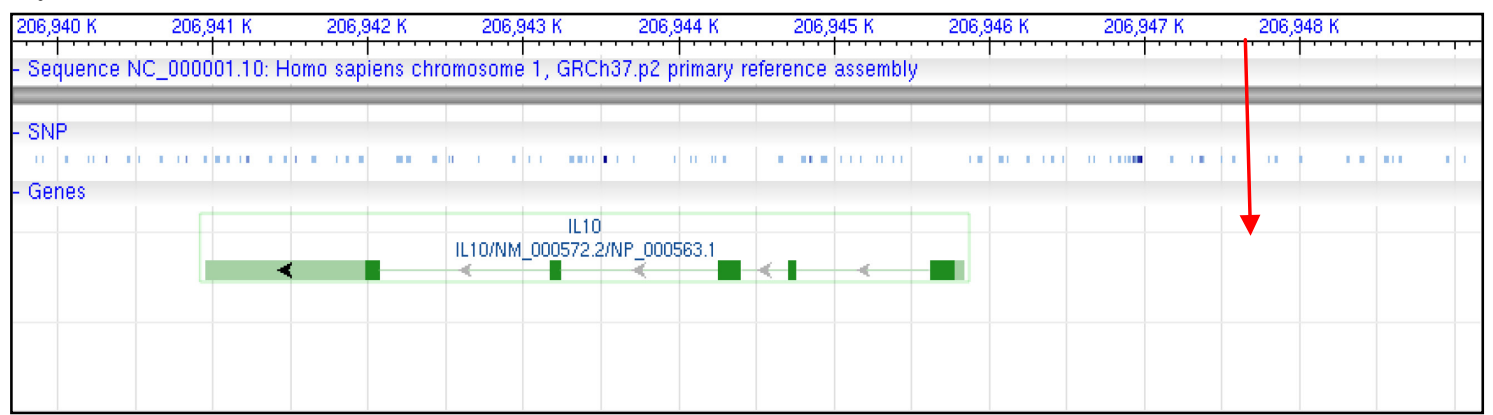

Figure 2-1. Promoter SNP Locations.

Note: The red down facing arrow in each image denotes the SNP location. Image A shows the IL- 6 gene and the location of rs1800795. Image B shows the IL-10 gene and the location of rs1800896. Images obtained from NCBI's new Sequence Viewer v.2.12. Available from: http://www.ncbi.nlm.nih.gov/SNP/. 
Table 2-3. HapMap 3 RS1800795 Genotype and Allele Frequencies.

\begin{tabular}{ccccc}
\hline Population & Number & CC & CG & GG \\
\hline CEU & 226 & 0.319 & 0.434 & 0.248 \\
ASW & 98 & 0 & 0.184 & 0.816 \\
YRI & 120 & 0 & 0 & 1.0 \\
\hline
\end{tabular}

Note: The ancestral allele is G. CEU represents Utah residents with Northern and Western European ancestry. ASW represents African ancestry from Southwest USA. YRI represents Africans from Sub-Sahara. Courtesy: National Human Genome Research Institute. 
Table 2-4. HapMap 3 RS1900896 Genotype and Allele Frequencies.

\begin{tabular}{ccccc}
\hline Population & Number & AA & AG & GG \\
\hline CEU & 226 & 0.212 & 0.513 & 0.274 \\
ASW & 98 & 0.449 & 0.429 & 0.122 \\
YRI & 120 & 0.531 & 0.389 & 0.080 \\
\hline
\end{tabular}

Note: The ancestral allele is A. CEU represents Utah residents with Northern and Western European ancestry. ASW represents African ancestry from Southwest USA. YRI represents Africans from Sub-Sahara. Courtesy: National Human Genome Research Institute. 
associated infections. The investigator performed a retrospective 6 week feasibility study in the ICU where this dissertation study subsequently occurred. ${ }^{24}$ Since cytokine measurements were not clinically available, the relationship of baseline C-reactive protein (CRP) to the development of HAI was examined. All patients admitted to the MICU/CICU for 6 weeks beginning 1/1/08 were screened for inclusion. All subjects with a CRP drawn within 24 hours of ICU admission were included. Baseline demographics, CRP, diagnoses, SIRS, APACHE II, MODS, infections, and invasive devices were recorded and all patient records were reviewed through ICU discharge for the development of HAI. Comparisons were made among those who did and did not develop HAI.

Among 69 patients admitted to the ICU during the study period, 27 patients had CRP levels within the first 24 hours. Among the 27 patients included in this older $(62.3$ $\pm 12.9)$, male $(96.3 \%)$ heterogeneous population, the mean APACHE II score was 17.8 $\pm 7.0 ; 25.9 \%$ required vasopressors, $59.6 \%$ had suspected or definitive baseline infections, $40.7 \%$ required mechanical ventilation, and the median baseline CRP level was $58.4 \mathrm{mg} / \mathrm{dL}$ with wide variation (range 3 - 548). Nine (33.3\%) patients developed HAI (total of 15 infections) with bacteremia and UTI being most common. There were no significant differences in survival, baseline CRP, APACHE II , SIRS, MODS, or hospital length of stay in those who did and did not develop NI. There was a trend $(p=0.10)$ towards development of a difference in ICU length of stay (10.4 vs. 4.4) in those who developed new HAI versus those who did not, respectively. Patients with baseline infections were more likely to develop new HAI 8 (88.9\%) compared to those who did not present with an infection $1(11.1 \%), p=0.04$. Although this was a small sample, it suggests increased susceptibility to new HAI in those who were admitted with sepsis. 


\section{CHAPTER 3. METHODS}

\section{Research Design}

This study was designed as a prospective observational study to evaluate the effects of baseline systemic inflammation as measured by cytokine levels on the development of HAIs in patients admitted to the ICU with sepsis or suspected sepsis. Baseline pro-inflammatory cytokine, anti-inflammatory cytokine, and their ratios, along with a common single nucleotide polymorphism for each cytokine tested were examined among patients with sepsis who did and did not develop subsequent HAIs. Approval for human subject's research was obtained from the Memphis-Veteran's Affairs Medical Center (VAMC) Institutional Review Board (IRB) and University of Tennessee Health Science Center (UTHSC) IRB, as well as the VAMC's Research and Development committee.

\section{Study Population}

All patients admitted to the Veterans Affairs Medical Center's Medical Intensive Care Unit (MICU) were systematically screened for sepsis at admission. A sample size of 78 subjects was required to detect a $30 \%$ difference $(40 \%$ in those with high systemic inflammation versus $10 \%$ in those with low systemic inflammation) in development of at least one HAI during ICU stay (up to ICU day 28). This calculation was estimated for patients with high baseline levels of systemic inflammation (pro-inflammatory cytokine level within $4^{\text {th }}$ quartile) versus patients without high levels of systemic inflammation (pro-inflammatory cytokine level not within $4^{\text {th }}$ quartile) with an $80 \%$ power given a $3: 1$ ratio.

\section{Inclusion Criteria}

The following two conditions were inclusion criteria:

1. Presence of Sepsis within 72 hours of admission to the ICU: Sepsis is defined as systemic inflammatory response syndrome (SIRS) plus an identified or suspected infection. SIRS is defined as at least two of the following: abnormal temperature $\left(>38^{\circ} \mathrm{C}\right.$ or $<36^{\circ} \mathrm{C}$ ), minimum heart rate ( $>90$ per minute), elevated respiratory rate ( $>20$ per minute) or a $\mathrm{PaCO}_{2}$ of $<32 \mathrm{mmHg}$, and an abnormal white blood cell count ( $>12,000$ or $<4,000$ cells $\mu \mathrm{L}^{-1}$ ) or bands $>$ $10 \%$.

2. Age 18 or older. 


\section{Exclusion Criteria}

The following four conditions were exclusion criteria:

1. Receiving Immunosuppressive agents such as chemotherapy or greater than 1 $\mathrm{mg} / \mathrm{kg}$ of prednisone or equivalent dose within the past 3 months.

2. Other Immunosuppression: AIDS.

3. Liver Failure defined as Child Pugh category $\mathrm{C}$ or higher.

4. Lack of informed consent.

\section{Procedures}

This section includes study related procedures.

\section{Summary of Procedures}

A summary of all study procedures over time is included in Table 3-1. Procedures included screening, informed consent, data collection, specimen collection, and specimen analysis.

\section{Screening}

Efforts were made to screen all patients for inclusion and exclusion criteria who were admitted to the medical intensive care unit (MICU). Rounds were systematically made in the MICU and the principle investigator interacted with the MICU medial team, nursing staff, and reviewed records to determine if patients met eligibility. A waiver of informed consent was obtained from the IRB to allow for screening of patient records for eligibility. Patient records included their electronic chart, nursing flowsheets, x-ray films, and other pertinent reports needed to determine eligibility. The principle investigator completed training in human subject's protection prior to initiation of this research.

\section{Informed Consent}

Subjects who met inclusion criteria and had no exclusion criteria were approached for informed consent. When subjects were too ill to provide their own informed consent, then a legally authorized representative/surrogate decision maker was sought for informed consent. All legally authorized representatives (LAR) who provided informed consent met requirement established by VAMC-IRB (see Appendix D, Informed Consent). The LAR designation used for this study was adopted from Tennessee State Law; however, a new designation has recently been adopted based on the latest release of the Veteran's Affairs Research Handbook which further limits LAR in regards to HIPPA consent for research. 
Table 3-1. Study Procedures.

\begin{tabular}{|c|c|c|c|}
\hline \multirow[b]{2}{*}{ Procedures } & \multicolumn{3}{|c|}{ Days } \\
\hline & 1 & $2-28$ & $\begin{array}{c}\text { ICU Discharge } \\
\text { (or Day 28) }\end{array}$ \\
\hline Screening for inclusion/exclusion & $\mathrm{x}$ & & \\
\hline Informed consent & $\mathrm{x}$ & & \\
\hline Data collection & & & \\
\hline Sepsis source and medical history & $\mathrm{x}$ & & \\
\hline APACHE II & $\mathrm{x}$ & & \\
\hline Health care associated infection & $\mathrm{x}$ & $\mathrm{x}$ & $\mathrm{x}$ \\
\hline Invasive devices & $\mathrm{x}$ & $\mathrm{x}$ & $\mathrm{x}$ \\
\hline Antibiotics, SIRS score, vital signs & $\mathrm{x}$ & $\mathrm{x}$ & $\mathrm{x}$ \\
\hline Clinical and lab assessments & $\mathrm{x}$ & $\mathrm{x}$ & $\mathrm{x}$ \\
\hline HAI preventive strategies & $\mathrm{x}$ & $\mathrm{x}$ & $\mathrm{x}$ \\
\hline Adverse events & $\mathrm{x}$ & $\mathrm{x}$ & $\mathrm{x}$ \\
\hline Plasma collection for cytokines & $\mathrm{x}$ & & \\
\hline Buccal swab collection for DNA & $\mathrm{x}$ & & \\
\hline Outcome assessment & & & $\mathrm{X}$ \\
\hline
\end{tabular}

Note: ICU denotes intensive care unit. SIRS denotes systemic inflammatory response score. 
As of March 2011, the only authorized LAR will include only individuals appointed under the Durable Power of Attorney for Health Care or a court-appointed guardian.

\section{Data Collection}

Baseline demographic variables were collected at ICU admission including age, race, and sex. Baseline sepsis (SIRS plus infectious cause) were collected in addition to descriptive variables surrounding baseline sepsis including the presence of organ failure (SOFA and MODS) and severity of illness (APACHE II).

Several predisposing factors were collected. Baseline plasma IL-6 and IL-10 levels were measured as well as their associated SNPS ra1800795 and rs1800896, respectively. Potential environmental exposures were also collected including invasive devices, nurse-patient ratios $>2: 1$ for any 4 hour period each day, and administration of blood products. The number and type of invasive device was recorded each day and the cumulative invasive devise score was recorded at the time of HAI and ICU discharge. Duration of ICU stay was collected.

Patients were followed through their ICU stay (or up to 28 days) for the presence of HAI based on CDC guidelines. Descriptive variables surrounding the first HAI were recorded including SIRS and organ failure (SOFA and MODS).

In addition to the primary variables a number of other variables were collected to better characterize the sample including vital signs, SIRS score, clinical triggers, all cultures, standard of care laboratory tests, standard of care CXRs, antibiotic use, use of steroids, and HAI preventive strategies.

Data were collected by the principle investigator using standardized forms which were updated during the data collection process (see Appendix E). Information about adverse events (primarily deaths) occurring during the study period were collected and reported to the IRB. The occurrence of life threatening arrhythmias and respiratory and cardiac arrests were also recorded. Data collection after ICU discharge was limited to 48 hours after ICU discharge for assessment of HAIs that could be attributed to ICU stay.

\section{Reliability and Validity of Common ICU Measures}

Vital signs are routinely recorded hourly in the ICU unless otherwise indicated. The values recorded by the critical care nurses during the course of standard patient care were reviewed. There was some variability in these measures and it was observed that respiratory rate was sometimes collected from the EKG respiratory lead (where it is captured and retained by the monitor) rather that from the ventilator display. There were instances of respiratory rates recorded that were lower than the ventilator set rate. When this occurred, the set ventilator rate was recorded as the minimum respiratory rate rather than the rate recorded on the flowsheet. Calculation of APACHE II requires the highest 
or lowest values within the first 24 hours, along with the worst lab values in this time period. The MICU uses bedside monitors with automated blood pressure cuffs which will provide for consistency in blood pressure readings. Arterial lines and central lines were routinely calibrated and maintained by bedside nurse per hospital policy. Standard quality control procedures in the hospital's laboratory include calibration of equipment at least every 8 hours and as needed. During the course of this study, Exergen's Temporal Scanner TAT-5000 Temporal Artery Thermometer became routine use in the MICU. There were no pilot studies to test the reliability or consistency of this method and it may have introduced some bias in temperature measurement, and therefore SIRS scores.

\section{Data Entry}

Participant records were examined prior to data entry to assure that data collection was complete. Clinical data were entered into a FilemakerPro ${ }^{\circledR}$ database. Prior to data analysis, data was exported to Excel, and all data was reviewed and cross checked for accuracy. Corrections were made to provide the most accurate dataset possible. Laboratory data (cytokine measures) were imported directly into Excel from the Luminex machine. Data from the LightCycler ${ }^{\circledR} 480$ Instrument were also imported to Excel for data analysis. Tests that did not meet specified conditions were repeated, and excel spreadsheets were updated to reflect additional results.

\section{Measurements}

This section provides detail for study measurements.

\section{Health Care Associated Infections}

A HAIs were defined as any infection occurring after day 3 through ICU discharge (or day 28 in participants with a prolonged ICU stay), excluding recurrent positive baseline infections. All participants were monitored with equal diligence for the development of HAIs daily through ICU discharge (or day 28 in participants with a prolonged ICU stay). Participants who were discharged from the ICU prior to day 28 were followed for 48 hours in the hospital. All infections that occur up to 48 hours after ICU discharge were also considered ICU related infections, just as infections occurring within 48 hours of hospital admission are considered community acquired. If a participant was readmitted to the ICU within 48 hours of ICU discharge, the patient was followed as though their ICU stay had been continuous.

All culture results during the study period were recorded. Developing an HAI during ICU stay was the primary outcome measure in this study. Each patient was classified as either having or not having a definitive HAI during ICU stay by culture criteria as defined below. Furthermore, the timing to development of first HAI was also recorded. The specific type of HAI and causative organism were recorded. Borderline 
HAIs were adjudicated by pulmonary and critical care consultation. HAIs were recorded in such a manner to allow for future incidence density calculations. The number and type of devices were recorded daily for each subject.

This study was implemented using the most recent HAI definitions by CDC Guidelines at that time. ${ }^{111}$ In November 2008, new HAIs guidelines replaced existing guidelines. ${ }^{112}$ These new guidelines implemented minor changes in HAI definitions but required that the new term "heath care associated infections" replace the prior term "nosocomial infections". Additionally, the names of common HAI have been updated to reflect the new guidelines. The operational definition for each type of infection did not change. Health care associated infections were defined by CDC Guidelines ${ }^{111}$ as follows, and terms were revised to meet current guidelines: ${ }^{112}$

- Bloodstream infection (formerly bacteremia) was defined by the presence of a microorganism cultured from blood which is not related to another site of infection. At least one blood culture is required to be positive, and when the identified organism is a potential skin contaminant such as coagulase-negative staphylococcus, two or more blood cultures must be positive.

- Pneumonia was clinically defined as the presence of fever $\left(>38^{\circ} \mathrm{C}\right)$, leukopenia $\left(<4,000 \mathrm{WBC} / \mathrm{mm}^{3}\right)$ or leukocytosis $(>12,000 \mathrm{WBC} / \mathrm{mm} 3)$, and new or worsening infiltrate on CXR (or the presence of consolidation or cavitation), and at least two of the following: (1) new onset of purulent sputum, or change in sputum of change increased respiratory secretions, or increased suctioning requirement, (2) New onset of worsening cough, or dyspnea, or tachypnea, (3) Rales or bronchial breath sounds, or (4) worsening gas exchange, increased oxygen requirements, or increased ventilation demand. Pneumonia was also definitively defined by positive bronchial alveolar lavage (BAL) with at least $10^{4} \mathrm{cfu} / \mathrm{ml}$.

- Urinary tract infection (UTI) can be either symptomatic or asymptomatic. Symptomatic UTI is defined as a positive urine culture with $>10^{5}$ microorganisms $/ \mathrm{ml}$ and one of the following: fever $\left(>38{ }^{\circ} \mathrm{C}\right)$; frequency; dysuria, loin pain; loin/suprapubic tenderness. A culture count of $>10^{3}$ microorgansims may be considered significant if obtained from a suprapubic puncture or in the presence of an antibiotic. It should be noted that many critical care patients may not be able to communicate their symptoms and steroids may results in failure to exhibit fever; therefore, culture counts of $>$ $10^{5}$ microorgansims in a urine culture obtained from an indwelling Foley catheter were considered to be urinary tract infections. Other infections of the urinary tract include presence of an abscess by direct observation during a surgical procedure.

- Sinusitis was defined by organisms cultured from the sinus cavity with the presence of radiographic changes. In addition, one or more of the following symptoms with no other recognized cause may be present: fever $\left(>38^{\circ} \mathrm{C}\right)$, 
pain or tenderness over the involved sinus, headache, purulent exudate, or nasal obstruction.

- A Cardiovascular system infection-VASC (formerly catheter-related infection) was defined as at least 15 colonies by semi-quatitative culture from an intravascular cannula tip in the presence of fever $\left(>38^{\circ} \mathrm{C}\right)$, pain, erythema, or heat at involved vascular size regardless of blood culture results.

Additionally, a catheter related infection can be diagnosed if purulent drainage was present at the vascular site. It should be noted that many critical care patients may not be able to communicate their symptoms and steroids may results in failure to exhibit fever.

In addition to infections that are definitive (evidence by positive cultures), it is possible that a patient may have had an infection that is not detected by culture. All patients were followed prospectively. In the absence of positive cultures, a high degree of clinical suspicion of infection which is treated and improves with an antibiotic was recorded. New fever in the ICU can be infectious or non-infectious, thus it is standard practice that any new fever is carefully investigated by the critical care team. ${ }^{54}$ All potentially non-definitive infections were reviewed with a critical care clinician. Primary analysis will include only definitive infections.

\section{Invasive Devices}

The presence of invasive devices was recorded daily through ICU discharge (or up to 28 days for participants with a prolonged ICU stay). Invasive devices include endotracheal tubes, central venous catheters, peripheral intravenous catheters, arterial catheters, chest tubes, nasal feeding tubes, Foley catheters, and other drains or catheters that have been inserted. A total daily invasive devise score as well as a cumulative daily score will be calculated for each patient. Each invasive devise will be given a score of 1 . For example, if a patient has three invasive devices for days 1-5, then only one device for days 6-14, then their daily score will be 3 for the first five days and 1 for subsequent days. On day 14 , the cumulative score will be $(3 * 5+1 * 9)=24$. A cumulative score for each day of the study will provide an estimate of their total exposure to invasive devices at any given time during the study period. This is a novel way to examine invasive devices and may be important since infections that occur early (day 5) will have less exposure to invasive devices than those that occur later. The invasive device score to be used in the regression model will be the invasive device score at the time of first HAI. In patients who do not develop any HAIs, the total ICU stay (up to day 28) invasive device score will be used in the regression model.

\section{Specimen Processing and Analysis}

Universal precautions were used during specimen collection, processing, and analysis. All specimens were collected at study entry. 


\section{Plasma Processing}

Blood was collected on each participant at baseline in $5 \mathrm{ml} \mathrm{ACD}$ tubes. The samples were placed on ice immediately and transported to the laboratory for processing as quickly as possible. A refrigerated centrifuge was used in the hospital Core Lab to spin the samples for 15 minutes at $2000 \mathrm{G}$ to separate the plasma from other blood components. Each plasma samples was then aliquoted into 2 equal portions and stored at -80 degrees until batch analysis. Samples were placed on dry ice when moving across campus prior to storage in the Crowe Building freezer, where all analyses were to be performed.

\section{Cytokine Analysis}

The cytokines for IL-6 and IL-10 were measured (pg/ml) in duplicate by Luminex (Luminex Corporation, Austin, TX) using Human Cytokine/ Chemokine Multiplex Kits (Millipore Corporation, Billerica, MA) according to manufactures instructions. In brief, previously frozen plasma samples were prepared by vortexing and certifuging to remove particulates before performing the assay. Beads were mixed according to instructions. A standard curve was prepared with the concentrations of $3.2 \mathrm{pg} / \mathrm{ml}, 16 \mathrm{pg} / \mathrm{ml}, 80 \mathrm{pg} / \mathrm{ml}$, $400 \mathrm{pg} / \mathrm{ml}, 2000 \mathrm{pg} / \mathrm{ml}$, and 10,000 pg/ml. The 96 well plate was prepared by adding 200 $\mu l$ of wash buffer to each well, shaking at room temperature for 10 minutes, and then vacuumed. Next, $25 \mu \mathrm{l}$ of standard each curve concentration, each control, and assay buffer were added to each well in duplicate as indicated. The plasma samples were then added to the appropriate sample wells $(25 \mu \mathrm{l})$ and serum matrix $(25 \mu \mathrm{l})$ added to each standard curve and control well. Next, $25 \mu \mathrm{l}$ of mixed beads were added to each well. The plate sealed and incubated overnight on the shaker at $4^{\circ} \mathrm{C}$. The next day, fluid was removed by vacuum and the plate was washed twice with $200 \mu$ of wash buffer and vacuumed. Next, $25 \mu \mathrm{l}$ of detection antibodies were added and the plate was placed on the shaker for one hour at room temperature. Next, $25 \mu \mathrm{l}$ of Streptavidin-Phycoerythrin was added to each well. The plate was sealed and placed on the shaker at room temperature for 30 minutes. The plate was then vacummed and washed twice with $200 \mu 1$ of wash buffer. The beads were then resuspended in $150 \mu \mathrm{l}$ of sheath fluid and analyzed on the Luminex machine.

Luminex software provides an automated data interpretation report with standard curves. It provide several report, including a bead count report, median florescent intensity (MIF), results, and average results for duplicates. Luminex software calculates the results by extrapolating MIF values on the standard curve. No reading can be accurately measured if below the lowest point on the standard curve $(3.2 \mathrm{pg} / \mathrm{ml})$ or above the highest point on the curve $(10,000 \mathrm{pg} / \mathrm{ml})$. When results occur outside of these detectable limits, the lowest or highest detectable value is substituted for statistical purposes. Results for each well were reviewed. According to the manufacturer instruction, data resulting from a cell with a bead count of at least 50 beads provides reliable results. In cases where the bead count was less than 50 or when the duplicate results were greater than 10 percent difference, the assay was repeated to increase the 
accuracy of the measurement. A total of four were batches run. Batch one (samples 1 - 39), batch two (samples 1 - 39), batch three samples 40 - 78), and batch four (repeat samples from batches two and three).

It was initially anticipated that only two kits would be needed to complete these measurements and two kits were ordered. There were multiple issues with batch one due to wells not vacumming and low bead counts. It was considered a test batch and none of the results were used. Two additional kits were ordered to assure that the same lot number was used, and the prior additional kit was retained to run repeat samples as needed. Batch 4 included duplicate samples from batch two and three that were needed to clarify the results as well as several additional duplicate samples to test reproducibility. Before the analysis was complete, the bead counts became extremely low and it was assumed that the Luminex needle was clogged. The system was flushed to assure that the needle was working properly. The plate was vacuumed and beads were resuspended with $150 \mu \mathrm{l}$ of sheath fluid, and the analysis for batch four was repeated. This time the bead counts for the first few wells (which previously had sufficient bead counts) were extremely low and the batch was discontinued. Limited results from batch four were available. Results and bead counts were carefully reviewed. A total of five cytokines were actually tested but only two are included in this dissertation study. It was a puzzling finding that some wells (from batches 2 and 3) which had sufficient bead counts for one cytokine but not another could have different results when retested. Each batch contained its own standard curve and controls. Perhaps this could be explained by the fact that the kit used for batch four had a different lot number and samples 1 - 39 had undergone one freeze thaw cycle. Most samples were measured in duplicate and averaged. Due to the inconsistent results of batch four, results from batch 2 and 3 were used primarily. A small number of values are based on single and not duplicate values.

\section{Reliability and Validity of Cytokine Measures}

The literature describes a high degree of variability among earlier generation cytokine results obtained from different manufacturers. Studies from the early 1990's found great variably in cytokine measurements depending on the type of fluid being analyzed and the assay used. ${ }^{113}$ Fahey et al. compared laboratories testing for cytokines and found both intra and inter laboratory variability. Several problems were identified before uniform results were obtained. ${ }^{114}$ The World Health Organization established cytokine standards to facilitate development of cytokine kits in research, and resulted in a dramatic reduction of the variation. ${ }^{115}$ It is recommended that when measuring cytokines, the same manufacturer's kit, methods, and lot number are used to enhance the internal validity of the study. Millipore reports precision percentage for inter-assay is $3.7-17.2$ and intra-assay is $4.6-13.8$. 


\section{Buccal Swab Collection for DNA}

The Catch-All ${ }^{\mathrm{TM}}$ Sample Collection Swabs from EPICENTRE Biotechnologies were used to collect buccal/cheek swabs. Specimens were collected by swabbing the inner aspect of the cheek and retaining the sample until ready for DNA extraction. All specimens were collected without the presence of tea or coffee for at least one hour. Yield is directly correlated with the starting amount of buccal cells; therefore, swabs were collected in duplicate due to the concern of low yield among patients receiving mechanical ventilation in which access to the mouth is obscured by a large bite block. Specimens were allowed to dry at room temperature, returned to the collection sleeve, temporarily stored at room temperature, and then transferring to a -20 degree freezer in the Crowe building. The protocol allows for storage at room temperature for up to one week. Several specimens were stored in a locked cabinet for 24 to 48 hours prior to freezing.

\section{DNA Extraction}

The BuccalAmp ${ }^{\text {TM }}$ Rapid DNA Extraction Kit was used to extract DNA according to the manufactures instructions and to prepare DNA for PCR amplification assays. In brief, frozen buccal swabs were allowed to thaw at room temperature. Tubes containing Quick DNA Extraction Solution (stored at -20 degrees) was allowed to thaw at room temperature. One of these tubes was then labeled for each patient. The tip of each buccal swab was gently rotated 10 times in a tube containing Quick DNA Extraction Solution. The swab was carefully removed while rotating and pressing on the side of the tube to prevent solution loss. The top was secured tightly on each tube, and then the tubes were vortexed for 10 seconds, incubated at $65^{\circ} \mathrm{C}$ for 1 minute, vortexed for 15 seconds, incubated at $98^{\circ} \mathrm{C}$ for 2 minutes, and then vortexed for 15 seconds. This process was done in batches as samples accumulated. A random sample $(n=6)$ was tested by spectrophotometry to assure the presence of DNA by examining 260/280 ratios, and then samples were stored at -70 degrees until ready for genetic testing. In the random sample 5/6 samples contained DNA. All samples were taken to the MRC to test the DNA quantity by NanoDrop but after checking several samples it was discovered that the proprietary reagents in the rapid extraction kits interfered with these results. According to manufactures instructions, SNP analysis can be performed without quantization or purification using this method.

\section{DNA Analysis}

SNP analysis was performed by real-time polymerase chain reaction (PCR) using the LightCycler ${ }^{\circledR} 480$ Instrument, located in UTHSC's Molecular Resource Center (MRC). TaqMan ${ }^{\circledR}$ SNP Genotyping Assays, Human, SM (40X) for rs1800795 and rs 1800896 from Applied Biosystems (Life Technologies, United States, 2006) contained florescent reporter tags (VIC and FAM) to determine alleles 1 and 2, respectively. Table 3-2 includes SNP primer details. A context sequence is given for proprietary primers. 
Table 3-2. SNP Primer and Reporter Details for Genotyping.

\begin{tabular}{lcc}
\hline Cytokine & IL-6 & IL-10 \\
\hline RS number & rs1800795 & rs1800896 \\
Forward Primer & CGACCTAAGCTGCACTTTTCC & \\
Reverse Primer & GGGCTGATTGGAAACCTTATT & $\cdot$ \\
\multicolumn{3}{c}{ AAGATTG } \\
VIC Reporter & CCTTTAGCAT[G]GCAAGAC & \\
Sequence & & $\cdot$ \\
FAM Reporter & CCTTTAGCAT[C]GCAAGAC & \\
Sequence & & $\cdot$ \\
Context & & \\
Sequence & & TCCTCTTACCTATCCCT \\
& ACTTCCC[T/C]TCCCA \\
& AAGAAGCCTTAGAGT \\
VIC Reporter & Targets C & GTTG \\
FAM Reporter & Targets G & Targets A \\
X Allele & GG & Targets G \\
Y Allele & CC & GG \\
Both Alleles & GC & AA \\
\hline
\end{tabular}

* Roche LightCycler $\AA^{8} 80$ Software reports describe alleles as Allele X, Allele Y, or Both Alleles. Allele X binds to fluorescent probes FAM at 483-533 nm, Allele Y binds to fluorescent probes VIC/HEX/Yellow555 at 523-568 nm. 
Roche LightCycler ${ }^{\circledR} 480$ Probe Master Mix and PCR plates were obtained from the MRC. A Master Mix was specifically made for each analysis which contained the Genotype Assay, Probe Master Mix, and PCR-grade water. The Genoype Assay was adjusted to a 20x concentration in the Master Mix. The total volume of each Master Mix depended on the number of reactions. The final reactions volume was $10 \mu \mathrm{l}$ and contained $1 \mu \mathrm{l}$ of unpurified DNA, $0.25 \mu \mathrm{l}$ of the Genotype Assay, $5.0 \mu 1$ TaqMan Master Mix, and 3.75 $\mu$ l PCR-grade water. Real-time PCR was performed according to manufacturer's instructions. Standard PCR methods were used. A negative control using PCR-grade water rather than DNA was included in each experiment. PCR included one preincubation period $\left(1\right.$ cycle at $\left.95^{\circ} \mathrm{C}\right)$, incubation $\left(45\right.$ cycles at $95^{\circ} \mathrm{C}, 62^{\circ}$, and $\left.72^{\circ} \mathrm{C}\right)$, and cooling period $\left(1\right.$ cycle at $\left.40^{\circ} \mathrm{C}\right)$. The primer target temperature is typically set approximately $5^{\circ} \mathrm{C}$ below the primer melting temperature (Tm); however, the Applied Biosystem's TaqMan ${ }^{\circledR}$ SNP Genotyping Assays are all optimized for $60^{\circ} \mathrm{C}$. If additional optimization is required, the manufacturer recommends either shortening cycle time to 40 or increasing the temperature to $62^{\circ} \mathrm{C}$.

\title{
Statistical Analysis
}

Data were analyzed with SAS (Version 9.1) using standard statistical techniques, including chi-square, student's t-test, correlations, and Cox regression modeling. Univariate testing was performed on all continuous variables, and variables not normally distributed were either log transformed to achieve a normal distribution or nonparametric tests were performed. Chi-square tests with expected cell counts less than five were analyzed using Fisher's Exact test. An alpha level of 0.05 was considered statistically significant. Each aim includes pre-specified research questions and assumes complete data for the primary variables of interest.

\begin{abstract}
Aim 1
This aim investigates whether baseline protein expression levels of proinflammatory cytokines, anti-inflammatory cytokines, or their ratios influence the development of subsequent HAI in patients with sepsis.

Chi-square $\left(\chi^{2}\right)$ tests were performed to test the proportions of patients with and without high systemic inflammation ( $4^{\text {th }}$ quartile, IL- 6 vs. other quartiles) who develop one or more HAI. $\chi^{2}$ tests were performed to test the proportions of patients with and without high systemic inflammation ( $4^{\text {th }}$ quartile, IL-10 vs. other quartiles) who develop one or more HAI. The ratio of IL-6:IL-10 were calculated and each participant categorized based on their ratio. A ratio greater than 1 indicates a more prominent proinflammatory response, a ratio less than one indicates a more prominent antiinflammatory response. Student's t was performed to determine prominent inflammatory response among those who do and do not develop one or more HAI. Mean cytokine
\end{abstract}


levels (IL-6, IL-10) and their ratio (IL-6:IL-10) will be compared using student's t among participants who do and do not develop a HAI during their ICU stay.

1.1 Does an exaggerated pro-inflammatory response influence subsequent HAI development in patients with sepsis?

1.2 Does an exaggerated anti-inflammatory response influence subsequent HAI development in patients with sepsis?

1.3 Do the ratios of pro-inflammatory and anti-inflammatory cytokines influence subsequent HAI development in patients with sepsis?

1.4 Describe baseline cytokine patterns among patients with sepsis who do and do not develop subsequent HAI?

\begin{abstract}
$\operatorname{Aim} 2$
This aim investigates the variance in cytokine genes to determine if they influence levels of protein expression or development of HAI.

ANOVAs were performed to determine the cytokine levels for each genotype. Chi-square analysis were also used to compare differences in common polymorphisms among those with exaggerated inflammation $\left(4^{\text {th }}\right.$ quartile $)$ at baseline as well as among those who do and do not develop HAIs.

2.1 Is a particular IL-6 genotype associated with a higher level of IL-6?

2.2 Is a particular IL-10 genotype associated with higher levels of IL-10?

2.3 Is a particular IL-6 genotype associated with a higher rate of HAI development?

2.4 Is a particular IL-10 genotype associated with a higher rate of HAI development?

2.5 Is a particular haplotype associated with a higher rate of HAI development?
\end{abstract}

\begin{abstract}
$\operatorname{Aim} 3$
This aim investigates the effects of protein expression levels, genetic variation, and environment on development of HAI.

A series of Cox regression analyses was performed among those who did and did not develop HAIs during ICU stay (or up to 28 days in those with a prolonged ICU stay) controlling for a number of potentially confounding variables including age, race, sex, severity of illness (APACHE II), baseline cytokines, ICU length of stay, invasive device score, steroid use, and potential confounders. Questions 3.1-3.7 were answered by univariate testing. Questions 3.8 and 3.9 were answered by multivariate testing.
\end{abstract}


3.1. What is the risk ratio to predict development of HAI for each 10 point increase in APACHE II?

3.2. What is the risk ratio to predict development of HAI for each additional invasive devise?

3.3. What is the risks ratio to predict development of HAI given IL-6-174G genotype?

3.4. What is the risks ratio to predict development of HAI given IL-10 -1082G genotype?

3.5. What is the risk ratio to predict development of HAI for each 10 point increase in pro-inflammatory cytokine?

3.6. What is the risk ratio to predict development of HAI for each 10 point increase in anti-inflammatory cytokine?

3.7. What is the risk ratio to predict development of HAI for each 10 point increase in ratio of pro- to anti-inflammatory cytokine?

3.8. Which variables are the strongest predictors of HAI development?

3.9. What is the final regression model for HAI? 


\section{CHAPTER 4. RESULTS}

Study results are presented in this chapter. We begin with a description of study recruitment and baseline demographics including details surrounding the initial sepsis or suspected sepsis event. Next, we provide a detailed description among subjects developing HAI in this study. We then discuss several ICU outcome variables based on development of HAI and based on exaggerated or not exaggerated pro- or antiinflammatory response. Lastly, results are described by each specific aim.

\section{Recruitment}

Screening occurred over an 18 month period from February 2009 until July 2010. Recruitment details are shown in Figures 4-1 and 4-2. A total of 539 subjects were screened with 215 (39.9\%) meeting inclusion criteria. Among all patients meeting inclusion criteria, 105 (48.8\%) met study related exclusion criteria, 32 (14.9\%) had other reasons for exclusion, and $78(36.3 \%)$ were enrolled. Approximately seven patients were screened for each patient enrolled. Some of the other reasons for exclusion included improvement and early transfer out of ICU, death, prior study subjects, or out of the window when evaluated.

\section{Demographics and Baseline Infections}

Subject demographics and clinical characteristics are shown in Table 4-1. The study population consisted of older $(65.5 \pm 12.6)$ male $(97.9 \%)$ veterans who were admitted to the ICU primarily from the emergency room $(48.7 \%)$ or general medical ward $(30.8 \%)$ with sepsis as a primary or underlying condition. This population included a high percentage with co-morbidities $(93.6 \%)$ with less than half $(43.6 \%)$ having diabetes. This population had a high severity of illness given their high APACHE II (20.6 \pm 6.4 ) and organ failure scores. Fifty (64.1\%) subjects had at least two organ failures at baseline.

Characteristics of baseline infection findings at ICU admission are shown in Table 4-2 and a rank percentage of baseline organisms identified is shown in Table 4-3. Among all baseline cultures, Staphylococcus Coagulase Negative was the most common microorganism, followed by Pseudomonas aeruginosa and Escherica coli. The most common type of infections among the 99 baseline infections were pneumonia $(35.4 \%$ CAP or HACP) and urinary tract infection (31.3\%). All patients had been placed on empiric antibiotics for their definitive or suspected infections at baseline. No microorganisms were identified in $22(28.2 \%)$ subjects. There were similar numbers of subjects with gram positive and gram negative infections. Blood-stream infection accompanied $32.0 \%$ of identified infections, with no source identified in $2(3.4 \%)$ subjects with a blood stream infection. Hypothermia or hyperthermia was present in 58 subjects. Baseline 


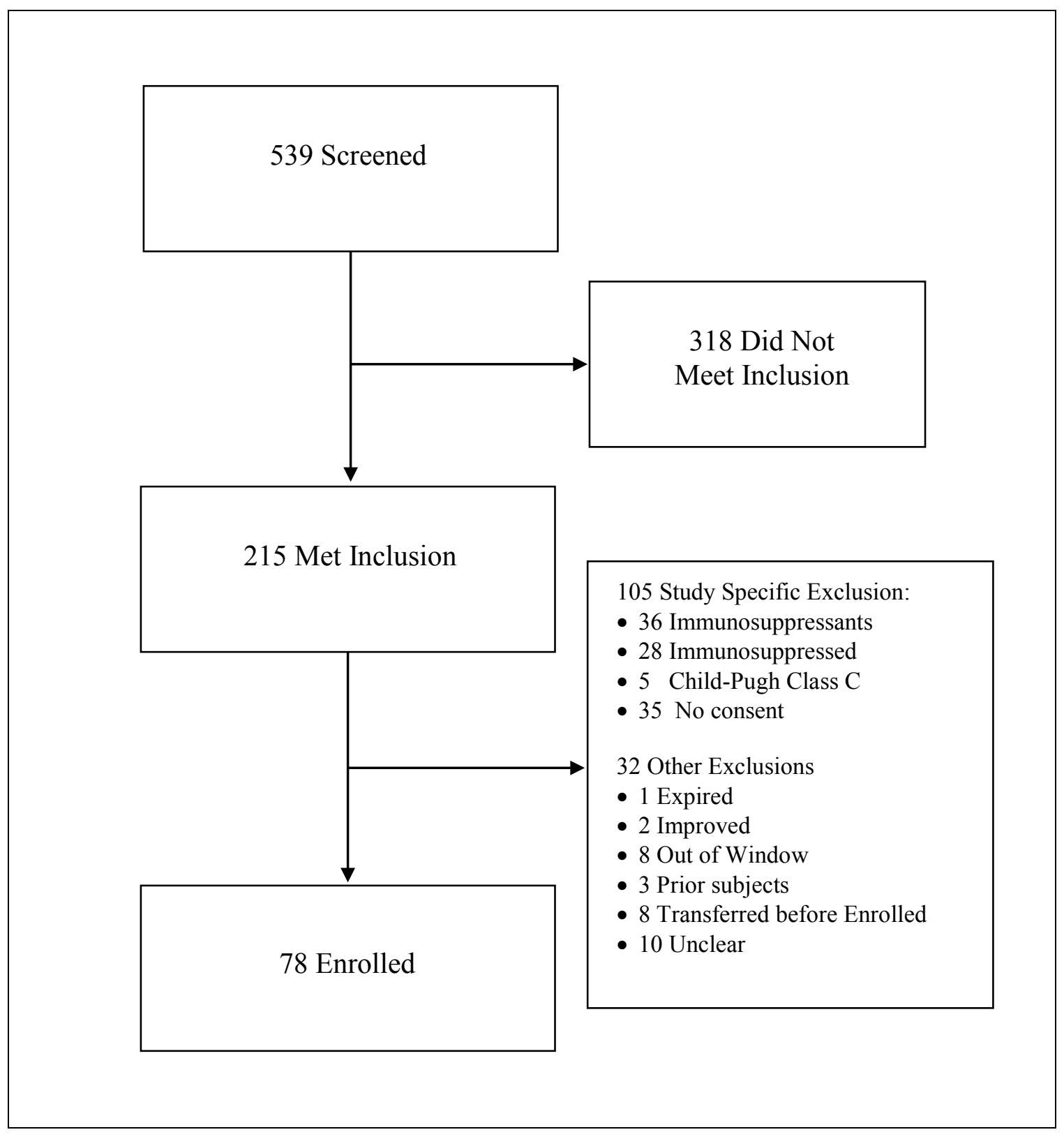

Figure 4-1. Subject Recruitment. 


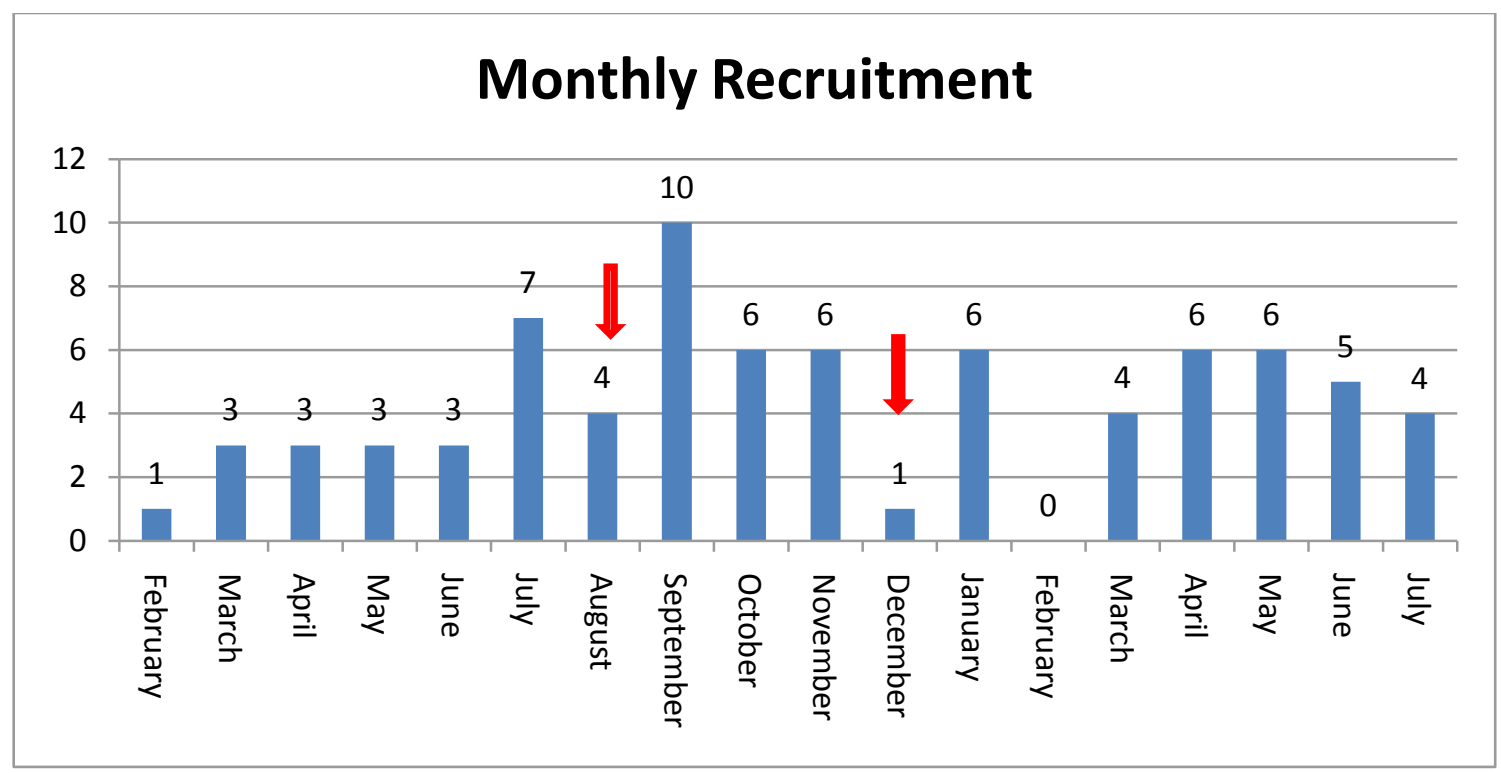

Figure 4-2. Monthly Subject Recruitment.

Legend: Down facing arrows denote protocols changes. The first arrow represents a protocol clarification of SIRS criteria by adding $>10 \%$ bands. The second arrow represents a protocol change to allow recruitment of MICU patients located in the SICU unit. 
Table 4-1. Demographic and Clinical Characteristics.

\begin{tabular}{|c|c|}
\hline Variables & $\begin{array}{l}\text { All Subjects } \\
\quad(\mathrm{n}=78)\end{array}$ \\
\hline Age (years) & $65.5 \pm 12.6$ \\
\hline \multicolumn{2}{|l|}{ Sex } \\
\hline Male & 74 (94.9) \\
\hline Female & $4(5.1)$ \\
\hline \multicolumn{2}{|l|}{ Race } \\
\hline Black & $30(38.5)$ \\
\hline White & $48(61.5)$ \\
\hline BMI* & $24.6(10.1)$ \\
\hline \multicolumn{2}{|l|}{ Admitted From } \\
\hline Emergency Room & $38(48.7)$ \\
\hline Another ICU & $3(3.8)$ \\
\hline Operating Room & $4(5.1)$ \\
\hline Spinal Cord Unit & $9(11.5)$ \\
\hline Ward & $24(30.8)$ \\
\hline Hospital Days Prior to ICU Admission† $\dagger$ & $1(3)$ \\
\hline Prior Hospitalization for More than 1 Week & $13(16.7)$ \\
\hline \multicolumn{2}{|l|}{ Primary Reason for ICU Admission } \\
\hline Sepsis or Severe Sepsis & $33(42.3)$ \\
\hline Septic Shock & $20(25.6)$ \\
\hline Respiratory Distress or Failure & $13(16.7)$ \\
\hline Other $\$$ & $12(15.4)$ \\
\hline Charlson Comorbidity Index $>0 \S$ & $73(93.6)$ \\
\hline Diabetes & 34 (43.6) \\
\hline Current Smoker & $21(26.9)$ \\
\hline Steroids Less than $1 \mathrm{mg} / \mathrm{kg} \|$ & $17(21.8)$ \\
\hline APACHE II & $20.6 \pm 6.4$ \\
\hline Sequential Organ Failure Score & $6.9 \pm 3.9$ \\
\hline Multiple Organ Dysfunction Score & $1.9 \pm 1.2$ \\
\hline Two or More Organ Failures & $50(64.1)$ \\
\hline
\end{tabular}

Note: Data are reported as mean \pm standard deviation, median (interquartile range), or count (percentage).

* BMI denotes body mass index. BMI range 11.0 - 72.6.

$\dagger$ Hospital days prior to ICU admission are reported as median (IQR), range 0 - 260.

There were 22 (28.2\%) patients who were in the hospital greater than 3 days and 4 (5.1\%) who were hospitalized for more than 28 days.

$\$$ Other reasons for ICU admission include: 3 post code, 2 non ST elevation myocardial infarctions, 2 gastro-intestinal bleeding, 1 congestive heart failure, 1 diabetic ketoacidosis, 3 post-op (carotid endarterectomy, knee replacement, gastric-tube placement with peritonitis).

$\S$ The Charlson Comorbitidy Index includes 19 medical conditions with weighted scores ranging from 1 to 6 for each condition and a total possible score of $0-37$. $^{116}$ 
Table 4-1. Continued.

\| Two subjects transferred from the ward had no prior steroids given on the ward or recorded in BCMA, but it was discovered upon later chart review that they received 1 dose of steroids $>1 \mathrm{mg} / \mathrm{kg}$ in the ER. These subjects have been retained in all analysis following an intention to treat principle. 
Table 4-2. Baseline Infection Findings at ICU Admission.

\begin{tabular}{|c|c|}
\hline Variables & $\begin{array}{l}\text { All Subjects } \\
(\mathrm{n}=78)\end{array}$ \\
\hline Definitive Infections & $51(65.4)$ \\
\hline \multicolumn{2}{|l|}{ Type of Infection* } \\
\hline Primary Bacteremia & 2 \\
\hline Aspiration Pneumonia & 1 \\
\hline Community Acquired Pneumonia & 15 \\
\hline Health Care Associated Pneumonia & 20 \\
\hline Gastrointestinal or Intra-abdominal Infection & 10 \\
\hline Urinary Tract Infection & 31 \\
\hline Skin and Soft Tissue Infection & 12 \\
\hline Bone and Joint Infection & 2 \\
\hline CNS Infection & 2 \\
\hline Cardiovascular System Infection & 3 \\
\hline Malaria & 1 \\
\hline Number of Patients with 2 Sites of Infection & $21(26.9)$ \\
\hline Accompanying Bacteremia & $25(32.0)$ \\
\hline \multicolumn{2}{|l|}{ Type of Organism } \\
\hline Gram Positive & $19(24.3)$ \\
\hline Gram Negative & $18(23.1)$ \\
\hline Mixed Gram Positive and Gram Negative & $8(10.2)$ \\
\hline Fungal & $4(5.1)$ \\
\hline Mixed Fungal and Gram Positive & $3(3.4)$ \\
\hline Mixed Fungal and Gram Negative & $3(3.4)$ \\
\hline Mixed Fungal, Gram Positive, and Gram Negative & $1(1.3)$ \\
\hline No Organism Identified & $22(28.2)$ \\
\hline Hyperthermia (Temperature > 100.4) & $23(29.5)$ \\
\hline Hypothermia (Temperature < 96.8) & $35(44.9)$ \\
\hline SIRS & $3.1 \pm 0.7$ \\
\hline Temperature $\left({ }^{\circ} \mathrm{F}\right) \dagger$ & $98.5 \pm 3.0$ \\
\hline Respiratory Rate & $30.9 \pm 8.6$ \\
\hline White Blood Cell Count (Thousands) & $18.7 \pm 15.5$ \\
\hline Bands $t$ & $22.2 \pm 16.3$ \\
\hline Heart Rate Minimum & $92.0 \pm 19.4$ \\
\hline Minimum SBP & $85.6(23.3)$ \\
\hline Number of Fluid Boluses Required $\S$ & $3.3 \pm 3.3$ \\
\hline Use of Vasopressors Required & $25(32.0)$ \\
\hline $\mathrm{PaO} 2: \mathrm{FiO} 2$ Ratio & $210 \pm 140$ \\
\hline C-reactive Protein & $206.7 \pm 156.7$ \\
\hline Lactate & $3.2 \pm 2.7$ \\
\hline Hours to ICU admission & $4.6(6.0)$ \\
\hline Timing to First Antibiotics & $1.5(3.75)$ \\
\hline Surgical Treatment & $11(14.1)$ \\
\hline Other Treatment & $10(12.8)$ \\
\hline
\end{tabular}


Table 4-2. Continued.

\begin{tabular}{lc}
\hline Variables & $\begin{array}{c}\text { All Subjects } \\
(\mathrm{n}=78)\end{array}$ \\
\hline Conventional Mechanical Ventilation & $26(33.3)$ \\
Noninvasive Positive Pressure Ventilation & $8(10.3)$ \\
\hline
\end{tabular}

Note: Data are reported as mean \pm standard deviation, median (interquartile range), or count (percentage). The number of subjects is less than 78 for the following variables: $\mathrm{C}$ reactive protein $(n=65)$, lactate $(n=64)$, timing to ICU admission $(n=72)$, timing to first antibiotics $(\mathrm{n}=71)$.

* Type of infection includes all infections. There were a total of 99 suspected or definitive infections in 78 patients. The total number of infections in each category is shown. Gastrointestinal or intrabdominal infections include four with C-difficile colitis, five with peritonitis, and one with cholecystitis. Cardiovascular system infections include two with catheter related infection and one with endocarditis. Bone and joint infections include one with osteomylitis and one with septic arthritis. CNS infection includes one with encephalitis and one with meningitis. Urinary tract infection includes one case of toxic shock syndrome post urology procedure detecting an abscess.

$\dagger$ Temperature range from 93.5 to $104.3^{\circ} \mathrm{F}$.

+ Manual differential counts were only done in 51 subjects.

$\S$ Fluid bolus range from 0 - 14 liters. 
Table 4-3. Most Common Baseline Micro-organisms.

\begin{tabular}{cc}
\hline Organism & All Organisms Cultured \\
\hline Staphylococcus coagulase negative (G+cocci) & $26(10.9)$ \\
Pseudomonas aerugnosa (G-rods) & $20(8.4)$ \\
E. Coli (G-rods) & $20(8.4)$ \\
Klebsiella pneumoniae (G-rods) & $18(7.6)$ \\
Candida albicans (yeast) & $18(7.6)$ \\
Staphylococcus aureus (G+cocci) & $16(6.7)$ \\
Enterococcus spp. (G+cocci) & $16(6.7)$ \\
Probable Candida - no id (yeast) & $16(6.7)$ \\
Serratia marcescens (G-rods) & $6(2.5)$ \\
Clostridium spp. (G+rods) & $5(2.1)$ \\
\hline
\end{tabular}

Note: The number and percentage is shown. This table is based on all cultures

completed up to day 3. Only organisms present in at least 5 cultures are shown. There were $22(28.2 \%)$ subjects with no baseline organism identified. 
lactate and CPR levels were elevated. Subjects received an average of 3.3 liters of fluid resuscitation on the day of ICU admission. In general, fluids were given as a rapid bolus in response to hypotension but precise timing of fluid resuscitation was not recorded. The maximum volume received among participants was 14 liters in one patient, and there were 17 patients that did not require fluid resuscitation. Additionally, $25(32.0 \%)$ required vasopressors and $34(43.6 \%)$ required either conventional or non-invasive positive pressure ventilation at baseline. Median hours until first antibiotic and ICU admission were 1.5 and 4.6 hours, respectively.

\section{Description of First Health Care Associated Infection}

A total of 17 participants developed at least one HAI. Characteristics of the first HAI is shown in Table 4-4 and a rank percentages of organisms identified are shown in Table 4-5. Candida was responsible for 11 (64\%) of all identified first HAIs. There were three species of Candida identified (Candida albicans, Candida glabrata, Candida tropicalis) in addition to others not identified. The second most frequent organism was Staphylococcus Coagulase Negative. The primary type of infection was bloodstream infection (47.1\%) followed by pneumonia (23.5\%) and urinary tract infection (17.6\%).

Fever was present in approximately half (47\%) who developed HAI and the average SIRS score was $2.1 \pm 0.9$. Fluid bolus was required in $4(23.5 \%)$ with the volume ranging from 0.5 to 5 liters. Three participants (17.7\%) required vasopressors to support blood pressure, and $10(58.8 \%)$ had moderate organ failure in 2 or more organs. Lactate and CPR levels were elevated.

Measureable environmental factors included invasive devices, staffing ratios, and receipt of blood products. All participants (100\%) developing HAI had a central line, $88.2 \%$ had a Foley catheter, and $70.5 \%$ were receiving mechanical ventilation (median 8 days) at the time of first HAI. Three participants (17.7\%) had at least one eight hour period with a nurse-to-patient ratio of more than $2: 1$ within 48 hours preceding the first HAI. Seven participants $(41.2 \%)$ received blood products within 48 hours preceding the first HAI.

\section{Differences in Variables among Those Who Did and Did Not Develop HAI}

A summary of ICU outcomes is shown in Table 4-6 for all participants comparing those who did and did not develop HAI. There were several significant differences between those who did and did not develop HAI. Those who developed HAI had a higher number of invasive devices $(\mathrm{p}=0.04)$ at ICU discharge as well as a higher cummulative invasive device score $(p<0.0001)$. Those developeing HAI had a higher number of organs with at least moderate dysfuction $(2.4 \pm 2.3$ vs. $1.1 \pm 1.3, \mathrm{p}=0.04)$, required more use of vasopressors $(64.7 \%$ vs. $27.9 \%, \mathrm{p}=0.009)$ with more episodes of new shock $(p=0.003)$. Those developing HAI had an average ICU length of stay more 
Table 4-4. Description of First Health Care Associated Infection.

\begin{tabular}{lc}
\hline Variables & $\begin{array}{c}\text { Developed HAI } \\
(\mathrm{n}=17)\end{array}$ \\
\hline Day of ICU & $9(5)$ \\
Type of Infection & \\
Bloodstream Infection & $8(47.1)$ \\
Pneumonia & $4(23.5)$ \\
Urinary Tract Infection & $3(17.6)$ \\
Gastrointestinal System Infection & $1(5.9)$ \\
Cardiovascular System Infection-VASC & $1(5.9)$ \\
Polymicrobial Infection & $3(17.7)$ \\
Resistant Organism & $4(23.5)$ \\
Fever or Hypothermia Present & $8(47.0)$ \\
SIRS & $2.1 \pm 0.9$ \\
Temperature & $97.9 \pm 1.8$ \\
WBC & $18.5 \pm 9.5$ \\
Respiratory Rate & $30 \pm 8$ \\
Heart Rate Minimum & $75 \pm 15$ \\
Minimum SBP & $103.7 \pm 18.7$ \\
Number Requiring Fluid Bolus & $4(23.5)$ \\
Use of Vasopressors Required & $3(17.7)$ \\
PaO2:FiO2 ratio & $196 \pm 98$ \\
Sequential Organ Failure Assessment & $6.2 \pm 4.7$ \\
Multiple Organ Dysfunction Score & $2.2 \pm 1.6$ \\
C-reactive Protein & $146.2 \pm 157.9$ \\
Lactate & $1.8 \pm 1.5$ \\
CPIS Score & $3.5 \pm 2.4$ \\
Change in Antibiotics & $12(70.6)$ \\
Receiving Mechanical Ventilation & $12(70.6)$ \\
Duration of MV at Time of HAI* & $8(7)$ \\
Cumulative Invasive Devise Score at Time of HAI & $52.8 \pm 26.7$ \\
Invasive Devices Present: & \\
Endotracheal Tube & $10(58.8)$ \\
Tracheostomy & $2(11.7)$ \\
Central Line & $17(100 \%)$ \\
Foley Catheter & $15(88.2)$ \\
Any Patient to Nurse Ratio > 2:1 in Preceding 48 Hours & $3(17.7)$ \\
Received Blood Products in Preceding 48 Hours & $7(41.2)$ \\
\hline & \\
&
\end{tabular}

Note: Data are reported as mean \pm standard deviation, median (interquartile range), or count (percentage).

* Two patients had prior mechanical ventilation during this ICU stay but were off for more than 48 hours at the time of their HAI. One had received three days of MV and off five days when HAI developed; the other had been on MV five days, and off six days when HAI developed. 
Table 4-5. First HAI Micro-organisms (Ranked).

\begin{tabular}{|c|c|}
\hline Organisms & Rank and Percentage \\
\hline Candida albicans ${ }^{*}$ & $5(25)$ \\
\hline Staphylococcus coagulate negative ${ }^{\dagger}$ & $4(20)$ \\
\hline Candida glabrata & $2(10)$ \\
\hline Candida tropicalis & $2(10)$ \\
\hline Candida spp not identified ${ }^{\S}$ & $2(10)$ \\
\hline Acinetobacter baumanni ${ }^{\|}$ & $1(5)$ \\
\hline Clostridium difficile & $1(5)$ \\
\hline Escherica coli ${ }^{\uparrow}$ & $1(5)$ \\
\hline Klebsiella pneumoniae " & $1(5)$ \\
\hline Pseudomonas aeruginosa ${ }^{\#}$ & $1(5)$ \\
\hline
\end{tabular}

Candida species involved 11 (64\%) of all 17 identified first HAIs.

* 2 HCAP, 3 fungemia. One Candida albicans was mixed with Klebsiella pneumoniae in the BAL.

$\uparrow 2$ blood stream infections, 1 HCAP, 1 Cardiovascular system infection -VASC.

Two Coagulate Negative Staphylococcus were mixed, one with Candida albicans in the BAL and the other with Candida tropicalis in the blood

$\$ 2$ fungemia

$\S 2$ UTI, 1 with fungemia

$\|$ UTI

q HCAP

\# VAP 
Table 4-6. Differences in Variables among Those Who Did and Did Not Develop Health Care Associated Infections.

\begin{tabular}{lcccc}
\hline Variables & $\begin{array}{c}\text { All subjects } \\
(\mathrm{n}=78)\end{array}$ & $\begin{array}{c}\text { No HAI } \\
(\mathrm{n}=61)\end{array}$ & $\begin{array}{c}\text { Developed HAI } \\
(\mathrm{n}=17)\end{array}$ & $\begin{array}{c}\text { P } \\
\text { Value }\end{array}$ \\
\hline Any HAI Develop During ICU Stay & $17(21.8)$ & $0(0.0)$ & $17(100)$ &. \\
Number of Invasive Devices at Discharge & $3.3 \pm 1.3$ & $3.1 \pm 1.3$ & $3.8 \pm 1.3$ & 0.04 \\
Total Invasive Device Score at Discharge & $35.6 \pm 35.7$ & $23.9 \pm 23.0$ & $77.7 \pm 41.8$ & $<0.0001$ \\
SOFA Score at Discharge & $4.6 \pm 4.7$ & $4.1 \pm 4.2$ & $6.6 \pm 5.8$ & 0.10 \\
MODS Score at Discharge & $1.4 \pm 1.6$ & $1.1 \pm 1.3$ & $2.4 \pm 2.3$ & 0.04 \\
SIRS Score & $1.8 \pm 0.9$ & $1.9 \pm 1.0$ & $1.7 \pm 0.8$ & 0.35 \\
Mechanical Ventilation Duration (n 34$)$ & $8.0 \pm 6.5$ & $5.9 \pm 4.7$ & $11.1 \pm 7.6$ & 0.03 \\
Use of Vasopressors during ICU Stay & $28(35.9)$ & $17(27.9)$ & $11(64.7)$ & 0.009 \\
Duration of Vasopressor Use (Days) & $3.9 \pm 3.0$ & $3.9 \pm 3.1$ & $4.1 \pm 3.2$ & 0.87 \\
Any New Shock during ICU Stay & $9(11.5)$ & $3(4.9)$ & $6(35.3)$ & 0.003 \\
Blood Products during ICU Stay & $44(56.4)$ & $31(50.8)$ & $13(76.5)$ & 0.10 \\
ICU Survival & $64(82.1)$ & $52(85.3)$ & $12(70.6)$ & 0.17 \\
Discharge Destination &. & &. & 0.14 \\
$\quad$ Remained in ICU & $1(1.3)$ & $0(0.0)$ & $1(5.9)$ &. \\
$\quad$ Ward & $56(71.8)$ & $46(75.4)$ & $10(58.8)$ &. \\
$\quad$ Spinal Cord Unit & $7(9.0)$ & $6(9.8)$ & $1(1.5)$ &. \\
$\quad$ Morgue & $14(18.0)$ & $9(14.8)$ & $5(29.4)$ &. \\
Duration of ICU stay & $8.0 \pm 6.2$ & $6.4 \pm 4.0$ & $15.3 \pm 7.1$ & $<0.0001$ \\
Readmissions to ICU within 28 Days & $12(15.4)$ & $8(13.1)$ & $4(23.5)$ & 0.06 \\
Any Nurse Patient Ratio Greater than 2:1 & $14(18.0)$ & $11(18.0)$ & $3(17.7)$ & 1.00 \\
Steroids Use during ICU Stay & $40(51.3)$ & $26(42.6)$ & $14(82.4)$ & 0.005 \\
Insulin Use during ICU Stay & $57(73.1)$ & $42(68.9)$ & $15(88.2)$ & 0.13 \\
Received Blood Products during ICU Stay & $44(56.4)$ & $31(50.8)$ & $13(76.50$ & 0.09 \\
\hline
\end{tabular}

Note: Data are reported as mean \pm standard deviation, median (interquartile range), or count (percentage). 
than twice compared to those not developing HAI, and required a longer duration of mechanical ventilation. There was a higher rate of steroid use in those developing HAI compared to those who did not $(82.4 \%$ vs. $42.6 \%, p=0.005)$. Patients developing HAI also had a trend $(\mathrm{p}=0.06)$ towards higher ICU readmissions rate within 28 days as compared to those who did not develop HAI.

\section{Differences in Variables by Pro- and Anti-inflammatory Cytokine Quartiles among Those Who Did and Did Not Develop HAI}

A summary is provided in Table 4-7 comparing participants with high baseline systemic inflammation (fourth quartile) to participants without high baseline systemic inflammation (first-third quartiles) for both the pro-inflammatory cytokine IL-6 and the anti-inflammatory cytokine IL-10. Steroid use was the only significant difference among participants with a high anti-inflammatory response versus those without $(84.2 \%$ vs. $40.7 \%, \mathrm{p}=0.0013$ ). Among those with a high pro-inflammatory response there were several significant differences in comparison to those with a lower pro-inflammatory response. Organ dysfunction was higher, and there was a three fold higher number of participants requiring vasopressors $(73.7 \%$ vs. $23.7 \%, \mathrm{p}<0.0001)$. There was also a higher percentage of steroid use $(73.7 \%$ vs. $44.1 \%, p=0.03)$, as well as participants with arrythmias $(52.6 \%$ vs. $18.6 \%, \mathrm{p}=0.007)$. ICU Survival was similar for all participants regardless of baseline pro- or anti-inflammatory cytokine level.

\section{Cytokine and Genotype Measurements}

Cytokines were measured by Luminex in batches. Details are described in the methods section. The detection limits for each cytokine was $3.2 \mathrm{pg} / \mathrm{ml}$ and 10,000 pg/ml. A total of $5(6.4 \%)$ of IL-6 levels and $29(37.2 \%)$ of IL-10 levels were below the detection limit. In these cases, a surrogate of $3.2 \mathrm{pg} / \mathrm{ml}$ was used for statistical purposes.

DNA isolation occurred in batches during recruitment and after recruitment was complete. Genotyping was perfomed after study recruitment was complete using End Point Genotyping by Real-Time PCR. The LightCycler ${ }^{\circledR} 480$ software (version LCS480 1.5.0.39) by Roche (Mannheim, Germany) provided automated genotyping calls for each participant. A call of either Allele X, Allele Y, Both Alleles, Unknown, or Negative was provided for each of the 96 wells. Gentotypes for Allele X, Allele Y, and Both Alleles were described in Table 3.2. A negative call was received for the negative controls, empty wells, and for other possible reasons such as sample quality, inhibition, or primer/dimer formation. Unknown calls were received when the software was unable to determine the genotype.

A test run was performed to optimize the PRC reaction which included the first 10 participant's samples under conditions described in the methods section. Replication was not observed until 30 cycles during the test run; therefore, the cycle time was increased to 50 from 45 cycles based on expert guidance from a MRC scientists who assisted with 
Table 4-7. Differences in Variables Pro- and Anti-inflammatory Cytokine Quartiles.

\begin{tabular}{|c|c|c|c|c|c|c|}
\hline \multirow[b]{3}{*}{ Variables } & \multicolumn{3}{|c|}{ Pro-inflammatory Cytokine: IL-6 } & \multicolumn{3}{|c|}{ Anti-inflammatory Cytokine: IL-10 } \\
\hline & 1st-3rd & 4 th & $\mathrm{P}$ & 1st-3rd & 4 th & $\mathrm{P}$ \\
\hline & Quartiles & Quartile & Value & Quartiles & Quartile & Value \\
\hline Number of Subjects & 59 & 19 & & 59 & 19 & \\
\hline Any HAI Develop During ICU Stay & 11(18.6) & $6(31.6)$ & 0.55 & $12(20.3)$ & $5(26.3)$ & 0.43 \\
\hline Invasive Device Score at Discharge & $31.9 \pm 33.5$ & $47.0 \pm 40.1$ & 0.15 & $32.4 \pm 34.3$ & $45.6 \pm 39.1$ & 0.19 \\
\hline Invasive Devices at Discharge & $3.2 \pm 1.2$ & $3.5 \pm 1.7$ & 0.45 & $3.3 \pm 1.3$ & $3.4 \pm 1.6$ & 0.77 \\
\hline SOFA Score at Discharge & $3.7 \pm 3.6$ & $7.5 \pm 6.4$ & 0.02 & $4.2 \pm 4.3$ & $6.1 \pm 5.6$ & 0.19 \\
\hline MODS Score at Discharge & $1.1 \pm 1.3$ & $2.2 \pm 2.1$ & 0.04 & $1.2 \pm 1.6$ & $1.8 \pm 1.8$ & 0.23 \\
\hline SIRS Score & $1.8 \pm 0.9$ & $2.0 \pm 0.9$ & 0.50 & $1.9 \pm 1.0$ & $1.7 \pm 0.9$ & 0.66 \\
\hline Mechanical Ventilation Duration* & $7.1 \pm 5.6$ & $10.1 \pm 8.3$ & 0.32 & $7.1 \pm 5.6$ & $10.2 \pm 8.9$ & 0.29 \\
\hline Use of Vasopressors during ICU stay & $14(23.7)$ & $14(73.7)$ & $<0.0001$ & $18(30.5)$ & $10(52.6)$ & 0.08 \\
\hline Any New Shock during ICU stay & $6(10.2)$ & $3(15.8)$ & 0.68 & 7 (11.9) & $2(10.5)$ & 1.00 \\
\hline Blood Products during ICU stay & $32(54.2)$ & $14(73.7)$ & 0.60 & $33(55.9)$ & $11(57.9)$ & 0.88 \\
\hline ICU Survival & $50(84.8)$ & $14(73.7)$ & 0.31 & $49(83.1)$ & $15(79.0)$ & 0.74 \\
\hline Discharge Destination & & & 0.22 & & & 0.37 \\
\hline Remained in ICU & $0(0.0)$ & $1(5.3)$ & . & $0(0.0)$ & $1(5.3)$ & . \\
\hline Ward & $44(74.6)$ & $12(32.2)$ & . & $44(74.6)$ & $12(63.2)$ & . \\
\hline Spinal Cord Unit & $6(10.2)$ & $1(5.3)$ & . & $5(8.5)$ & $2(10.6)$ & . \\
\hline Morgue & $9(15.3)$ & $5(26.3)$ & . & $10(17.0)$ & $4(21.1)$ & . \\
\hline Duration of ICU stay & $7.4 \pm 5.6$ & $10.1 \pm 7.4$ & 0.16 & $7.5 \pm 5.7$ & $9.7 \pm 7.2$ & 0.24 \\
\hline Readmissions to ICU within 28 days & $9(15.3)$ & $3(15.9)$ & 0.26 & $8(13.6)$ & $4(21.1)$ & 0.40 \\
\hline Any Nurse Patient Ratio $>2: 1$ & $13(22.0)$ & $1(5.3)$ & 0.16 & $13(22.0)$ & $1(5.3)$ & 0.16 \\
\hline Steroids Use during ICU stay & $26(44.1)$ & $14(73.7)$ & 0.03 & $24(40.7)$ & $16(84.2)$ & 0.0013 \\
\hline Insulin Use during ICU stay & $43(72.9)$ & $14(73.7)$ & 0.95 & $46(78.0)$ & $11(57.9)$ & 0.09 \\
\hline Arrhythmias & $11(18.6)$ & $10(52.6)$ & 0.007 & $14(23.7)$ & $7(36.8)$ & 0.26 \\
\hline Cardiac Arrest & $13(22.0)$ & $7(36.8)$ & 0.23 & $15(25.4)$ & $5(26.3)$ & 0.94 \\
\hline
\end{tabular}

* Duration of mechanical ventilation includes data for 34 subjects who received mechanical ventilation, $\mathrm{n}=24$ versus 10 , respectively. 
LightCycler initial set-up and with calculations required for initial reaction volumes. The genotype results for IL-6 under these conditions included 32 Negatives and 5 Unknowns. Applied Biosystems technical support advised that although replication did not begin until 30 cycles, it is more appropriate for end point genotyping not to increase cycle time beyond 45 cycles. All samples were re-tested for IL-6 genotyping using the original test conditions (45 cycles) and resulted in only 2 undetermined genotypes ( 1 negative and 1 unknown call). These 2 missing genotypes were available from the initial test run.

Genotyping for IL-10 was performed as described in the methods section. The initial undetermined calls were 30 unknown and 1 negative. The reaction was optimized by increasing the temperature to $62^{\circ} \mathrm{C}$, reducing the number of unknowns to 7 . Manual calls were required for these 7 subjects: $2,22,27,31,50,58$, and 69 . These subjects were manually called heterozygotes based upon visual clustering and endpoint fluorescence values. All unknown calls had a confidence score less than 0.50 and endpoint fluorescence values were not significantly higher than each other. Although the software algorithm is proprietary, genotypes that were automatically called included an endpoint fluorescence of at least one or more fold higher than the lower endpoint fluorescence value. The endpoint fluorescence value for those called as heterozygous contained differences but they were generally much less than a fold difference.

\section{Genotype Allele Frequencies}

The allele frequencies are shown for rs1800795 and rs1800896 genotypes in Table 4-8 and 4-9, respectively. These tables also show allele frequencies for all subjects as well as white and black sepsis patients who do and do not develop HAI. Note that there were no significant differences between the percentage of blacks and white sepsis patients who do and do not develop HAI (23.3\% vs. $20.8 \%, \mathrm{p}=0.79)$.

For rs1800795, there were no significant allele frequency differences among those who do and do not develop HAI $(\mathrm{p}=0.59)$; however, when examining racial differences in genotype and controlling for those who do and do not develop HAI differences were noted. There was a significant difference in rs 1800795 genotype among black patients with sepsis who did not develop HIA compared to whites patients with sepsis who did not develop HAI $(\mathrm{p}=0.0056)$. Specifically, black patients had a lower CG $(17.4 \% \mathrm{vs}$. $42.1 \%)$ and higher GG $(82.6 \%$ vs. $42.1 \%)$ than white patients. There were no racial differences when comparing white and black sepsis patients who developed HAI ( $p$ $=1.0$ ).

For rs1800896, there were no significant allele frequency differences among those who do and do not develop HAI $(\mathrm{p}=0.16)$. There were no significant racial differences among those who did $(\mathrm{p}=1.0)$ and did not develop HAI $(\mathrm{p}=0.41)$. 
Table 4-8. RS1800795 Genotype and Allele Frequencies.

\begin{tabular}{lcccc}
\hline \multicolumn{1}{c}{ Population } & Number & CC & CG & GG \\
\hline All & 78 & 0.090 & 0.359 & 0.551 \\
White & 48 & 0.146 & 0.437 & 0.417 \\
$\quad$ HAI & 10 & 0.100 & 0.500 & 0.400 \\
$\quad$ No HAI & 38 & 0.158 & 0.421 & 0.421 \\
Black & 30 & 0.000 & 0.233 & 0.767 \\
$\quad$ HAI & 7 & 0.000 & 0.429 & 0.571 \\
$\quad$ No HAI & 23 & 0.000 & 0.017 & 0.826 \\
\hline
\end{tabular}


Table 4-9. RS1900896 Genotype and Allele Frequencies.

\begin{tabular}{lcccc}
\hline \multicolumn{1}{c}{ Population } & Number & AA & AG & GG \\
\hline All & 78 & 0.218 & 0.488 & 0.295 \\
White & 48 & 0.271 & 0.437 & 0.292 \\
$\quad$ HAI & 10 & 0.100 & 0.500 & 0.400 \\
No HAI & 38 & 0.316 & 0.421 & 0.263 \\
Black & 30 & 0.133 & 0.567 & 0.300 \\
HAI & 7 & 0.000 & 0.571 & 0.429 \\
No HAI & 23 & 0.174 & 0.565 & 0.261 \\
\hline
\end{tabular}




\section{Genotype Comparisons to HapMap 3 Reference Population}

There were differences in the genotype of sepsis patients as compared to a normal HapMap3 reference population (see Table 4-10). White (and not black) patients with sepsis had a significantly different $(\mathrm{p}=0.02)$ IL-6 genotype with higher GG $(41.7 \%$ versus $24.8 \%)$ and lower CC (14.6\% versus $31.9 \%)$ genotypes when compared to a normal reference. When examining IL-10 genotypes, black (and not white) patients with sepsis had a significantly higher GG (30.0\% versus $12.2 \%)$ and lower AA (13.3\% versus $44.9 \%$ ) genotype when compared to a normal reference.

\section{Baseline Cytokine Levels by Genotype and Haplotypes}

Plasma cytokine levels were right skewed and required log transformation for statistcs requiring a normal disturbution. Table 4-11 provides a summary of plasma IL-6 and IL-10 levels. Median IL-6 levels were higher than median IL-10 levels. Table 4-12 provides cytokine levels and their ratio for each genotype. Median IL-6 levels were highest among participants with the CC IL-6 genotype and also among participants with the GG IL-10 genotype. Median IL-10 levels were highest among participants with the AA IL-10 genotype and also among participants with the CG IL-6 genotype. Figures 4-3, 4-4, 4-5 and 4-6 provide box-plots of pro- and anti-inflammatory cytokines and their ratios by for IL-6 and IL-10 genotypes. Each figure contains two images. The top image shows the skewed data distribution prior to log transformation, and the lower image shows the log transformed data distribution.

\section{Aim 1}

The primary goal of aim one was to investigate whether baseline protein expression levels of pro-inflammatory cytokines, anti-inflammatory cytokines, or their ratios influence the development of subsequent HAI in patients with sepsis.

There was no significant difference in levels of pro-inflammatory cytokine, antiinflammatory cytokine, or their ratio among subjects who did and did not develop at least one HAI during their ICU stay. This aim was explored by comparing lower quartiles to the higher fourth quartile for proinflammatory cytokine IL-6 and anti-inflammatory cytokine IL-10, as well as comparing their ratios. Patients in the fourth quartile were considered to have an exaggerated inflammatory response as compared to those in other quartiles. Specifically, an exaggerated pro-inflammatory response was present in 6 $(31.6 \%)$ compared to $11(18.6 \%)$ participants without an exaggerated pro-inflammatory response who developed subsequent HAI. This difference was not significant $(p=0.55)$. Likewise, an exaggerated anti-inflammatory response was present in 5 (26.3\%) compared to 12 (20.3) participants without an exaggerated anti-inflammatory response who developed subsequent HAI. This difference was also not significant $(\mathrm{p}=0.43)$. There was also no significant difference in the log of proinflammatory to anti-inflammatory 
Table 4-10. Genotype Comparisons of IL-6 and IL-10 SNPs among Sepsis and HapMap Reference Population.

\begin{tabular}{|c|c|c|c|c|c|c|c|c|c|}
\hline Genotype & $\begin{array}{l}\text { HapMap } \\
(\mathrm{n}=324)\end{array}$ & $\begin{array}{l}\text { Sepsis } \\
(\mathrm{n}=78)\end{array}$ & $\begin{array}{c}\mathrm{P} \\
\text { Value }\end{array}$ & $\begin{array}{c}\text { HapMap: } \\
\text { Black } \\
(\mathrm{n}=98)\end{array}$ & $\begin{array}{c}\text { Sepsis } \\
\text { Black } \\
(\mathrm{n}=30)\end{array}$ & $\begin{array}{c}\mathrm{P} \\
\text { Value }\end{array}$ & $\begin{array}{l}\text { HapMap } \\
\text { White } \\
(\mathrm{n}=226)\end{array}$ & $\begin{array}{l}\text { Sepsis } \\
\text { White } \\
(\mathrm{n}=48)\end{array}$ & $\begin{array}{c}\mathrm{P} \\
\text { Value }\end{array}$ \\
\hline IL-6 & & & 0.01 & & & 0.60 & & & 0.02 \\
\hline $\mathrm{CC}$ & $72(22.2)$ & $7(9.0)$ & . & $0(0)$ & $0(0)$ & . & 72 (31.9) & $7(14.6)$ & 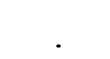 \\
\hline $\mathrm{CG}$ & $116(35.8)$ & $28(35.9)$ & . & $18(18.4)$ & $7(23.3)$ & . & $98(43.4)$ & $21(43.8)$ & . \\
\hline GG & $136(42.0)$ & $43(55.1)$ & . & $80(81.6)$ & $23(76.7)$ & . & $56(24.8)$ & $20(41.7)$ & . \\
\hline IL-10 & . & & 0.35 & 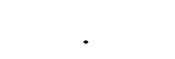 & & 0.002 & & & 0.54 \\
\hline $\mathrm{AA}$ & $92(28.4)$ & $17(21.8)$ & . & $44(44.9)$ & $4(13.3)$ & . & $48(21.2)$ & $13(27.1)$ & . \\
\hline $\mathrm{AG}$ & $158(48.8)$ & $38(48.7)$ & . & $42(42.9)$ & $17(56.7)$ & . & $116(51.3)$ & $21(43.8)$ & . \\
\hline GG & $74(22.8)$ & $23(29.5)$ & . & $12(12.2)$ & $9(30.0)$ & . & $62(27.4)$ & $14(29.2)$ & . \\
\hline
\end{tabular}

Note: The reference population is the HapMap 3. The HapMap 3 sample included in this comparison includes normal individuals from African ancestry in Southwest USA (black) and Utah residents with Northern and Western European ancestry (white). ${ }^{109}$ 
Table 4-11. Range of Plasma IL6 and IL10 Levels.

\begin{tabular}{lcccc}
\hline & & Inter-quartile & & \\
& Median $(\mathrm{n}=78)$ & Range & Minimum & Maximum \\
\hline IL-6 pg/ml & 167.9 & $48.4-345.5$ & 3.2 & 3862.4 \\
IL-10 pg/ml & 20.8 & $3.2-134.4$ & 3.2 & 2066.5 \\
Log IL-6 pg/ml & 5.1 & $3.9-5.8$ & 1.2 & 8.3 \\
Log IL-10 pg/ml & 3.0 & $1.2-4.9$ & 1.2 & 7.6 \\
\hline
\end{tabular}


Table 4-12. Median and Inter-quartile Range of Pro- and Anti-Inflammatory Cytokine Levels by Genotypes.

\begin{tabular}{cccc}
\hline Variables & $\begin{array}{c}\text { IL-6 Levels pg/ml } \\
(\mathrm{n}=78)\end{array}$ & $\begin{array}{c}\text { IL-10 Levels pg/ml } \\
(\mathrm{n}=78)\end{array}$ & $\begin{array}{c}\text { IL6:IL10 Ratio } \\
(\mathrm{n}=78)\end{array}$ \\
\hline Genotype: IL-6 SNP & & \\
CC & $260.2(90.4-406.8)$ & $8.8(3.2-36.3)$ & $10.2(6.1-81.3)$ \\
CG & $176.7(67.4-404.5)$ & $68.7(4.1-369.8)$ & $3.1(1.3-12.3)$ \\
GG & $119.0(28.0-293.1)$ & $16.4(3.2-132.8)$ & $4.9(1.5-17.9)$ \\
C Allele & $195.3(67.9-406.8)$ & $36.2(3.2-150.7)$ & $5.7(1.4-19.8)$ \\
\multicolumn{7}{l}{} & & \\
Genotype: IL-10 SNP & & \\
AA & $86.3(24.5-257.8)$ & $58.3(18.9-139.7)$ & $2.2(1.5-4.9)$ \\
GA & $163.4(43.5-293.1)$ & $3.2(3.2-60.7)$ & $9.2(1.4-27.1)$ \\
GG & $242.3(98.1-633.7)$ & $43.5(11.2-243.1)$ & $6.1(1.8-15.8)$ \\
A Allele & $157.1(29.9-284.6)$ & $10.5(3.2-106.7)$ & $4.7(1.4-20.9)$ \\
\hline
\end{tabular}




\section{Plasma IL-6 by Genotype}

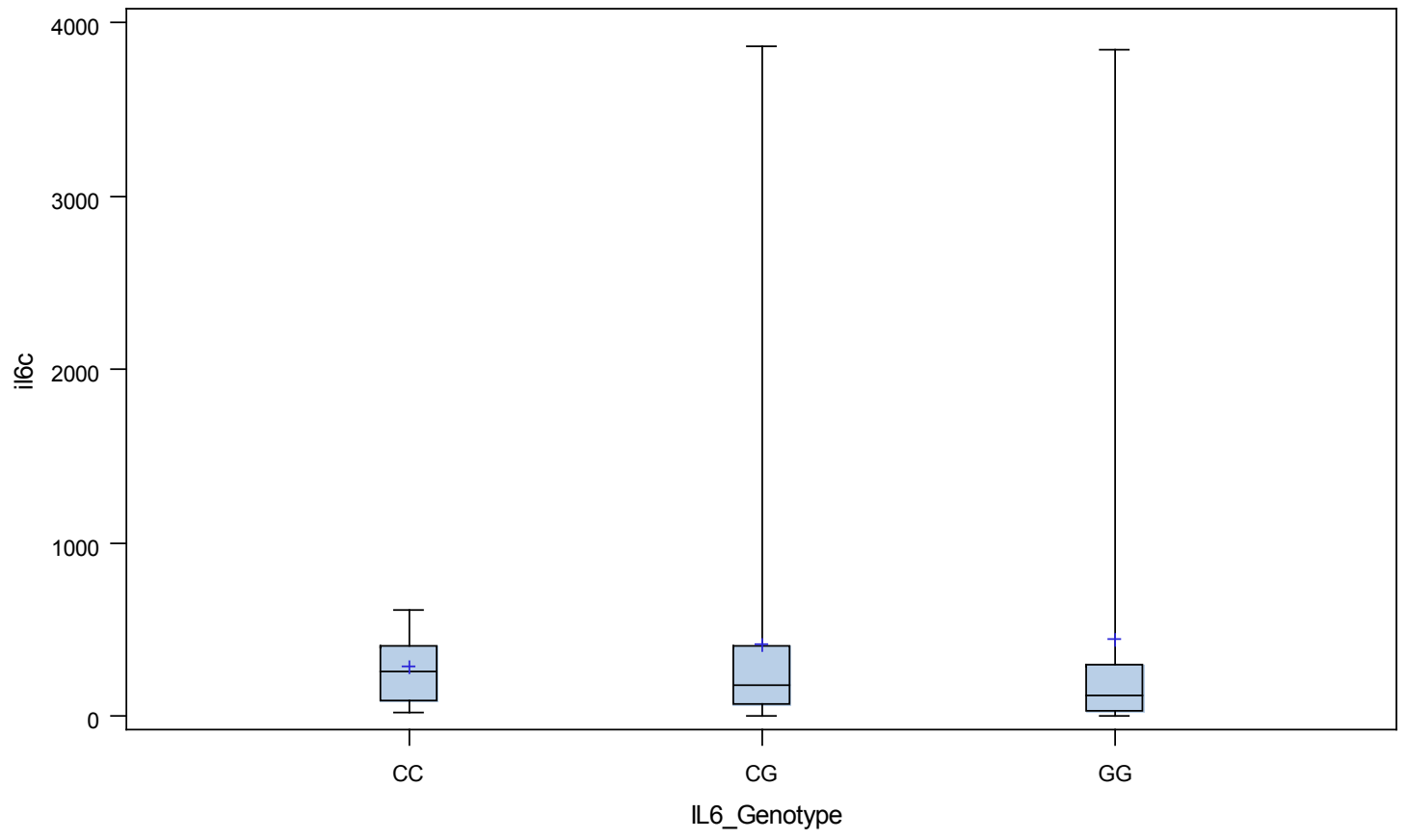

\section{Plasma Log IL-6 by Genotype}

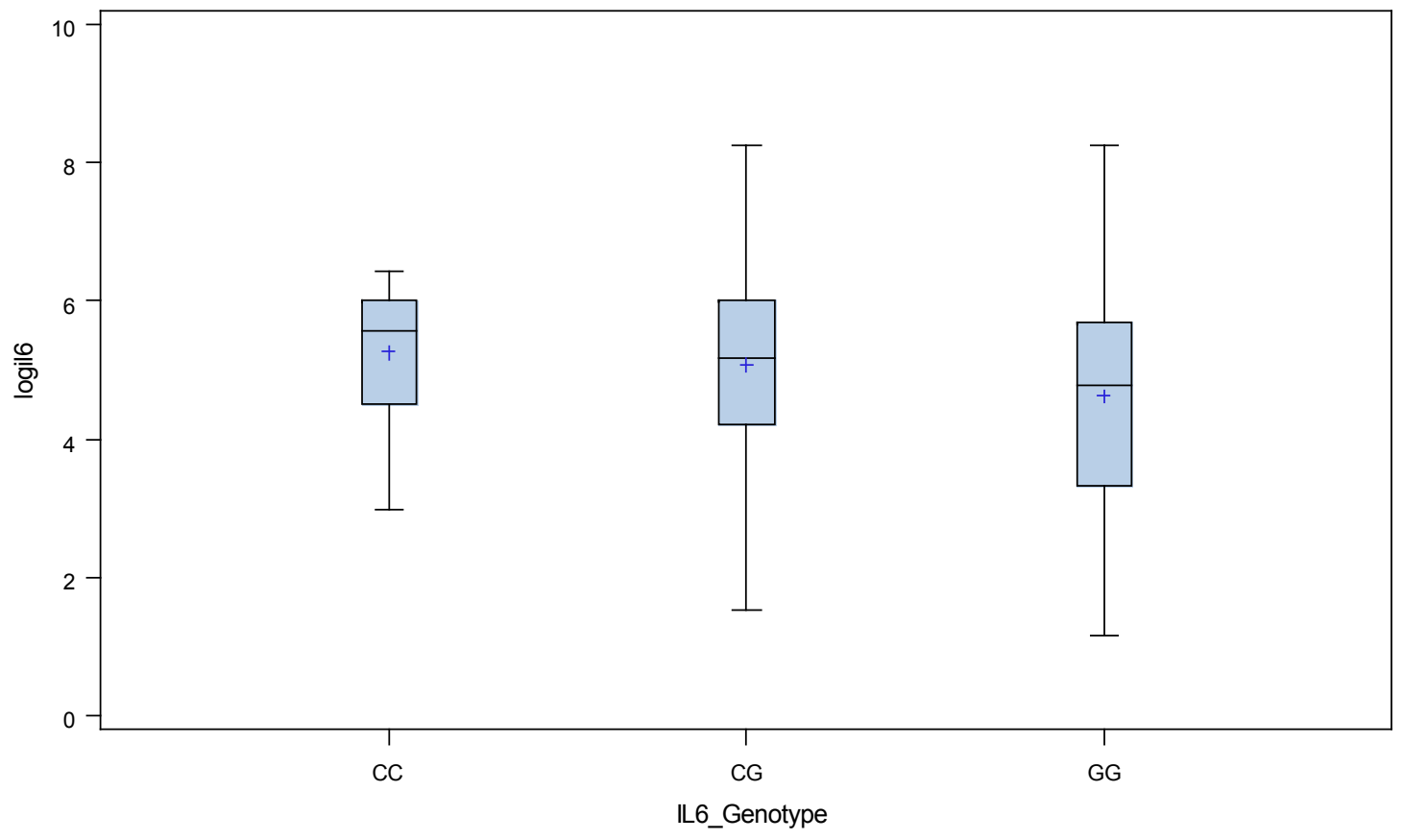

Figure 4-3. Box Plot of Plasma IL-6 levels by IL-6 Genotypes Pre and Post Log Transformation. 


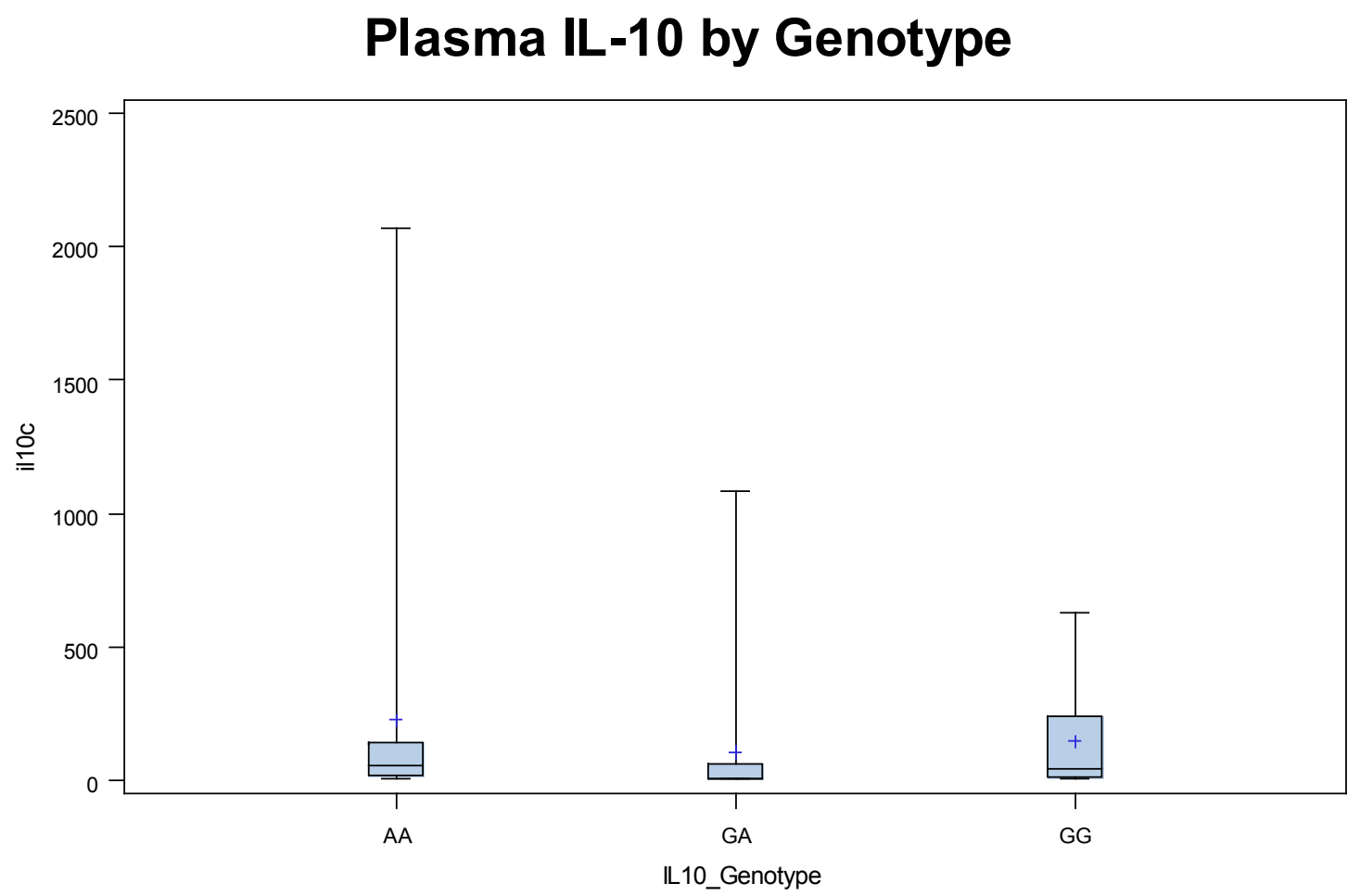

Plasma Log IL-10 by Genotype

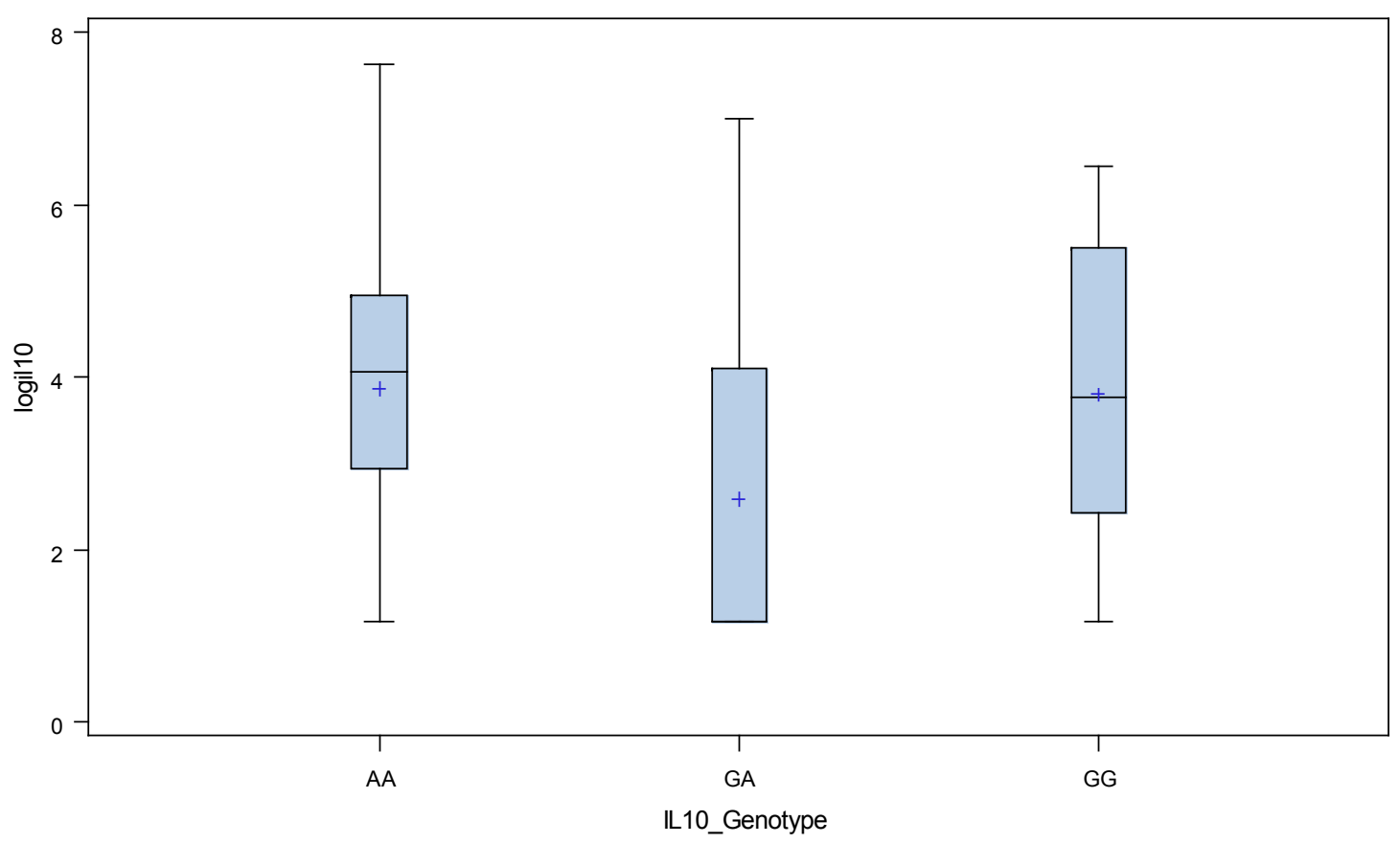

Figure 4-4. Box Plot of Plasma IL-10 levels by IL-10 Genotypes Pre and Post Log Transformation. 

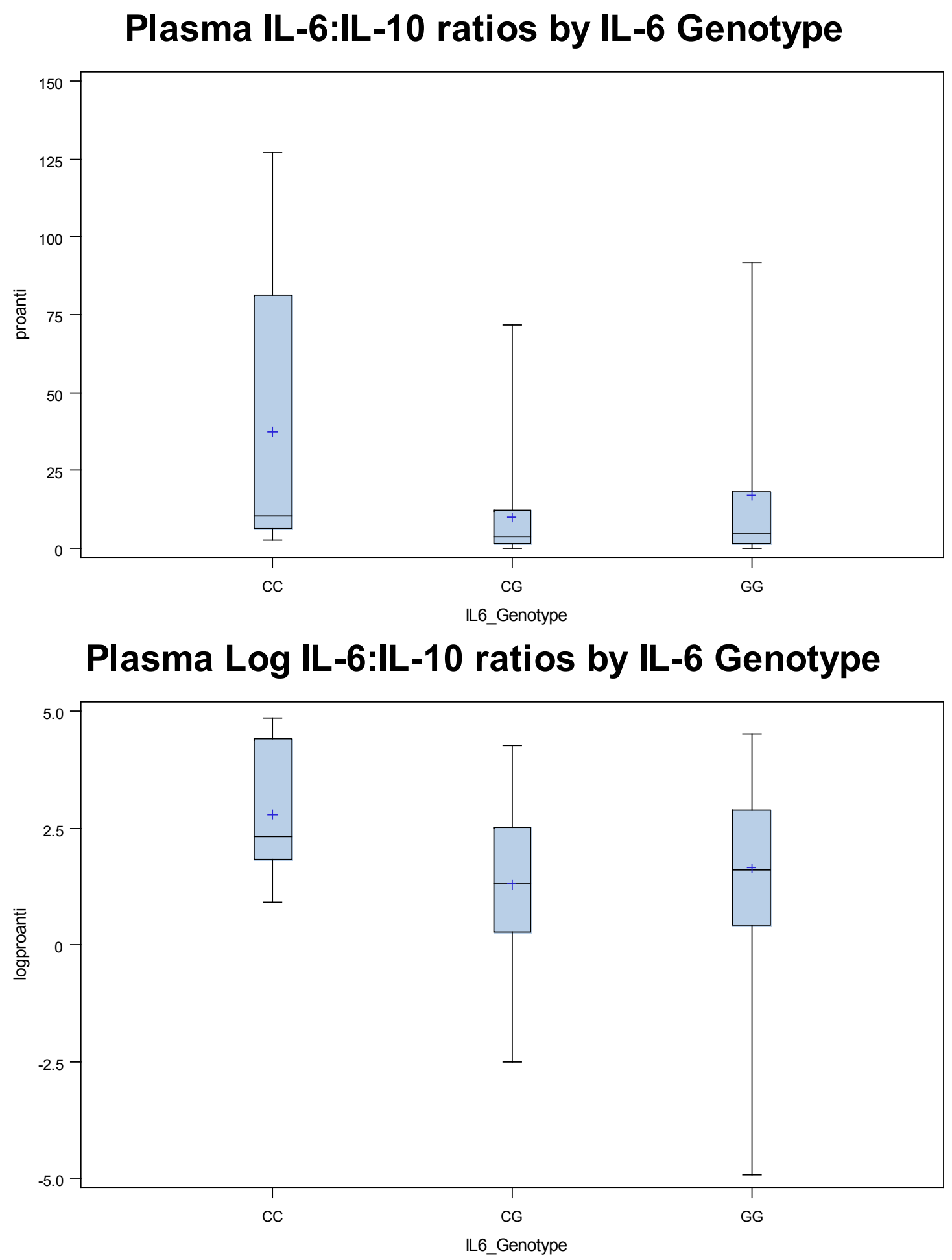

Figure 4-5. Box Plot of Plasma IL-6:IL10 ratios by IL-16 Genotypes Pre and Post Log Transformation. 


\section{Plasma IL-6:IL-10 by IL-10 Genotype}

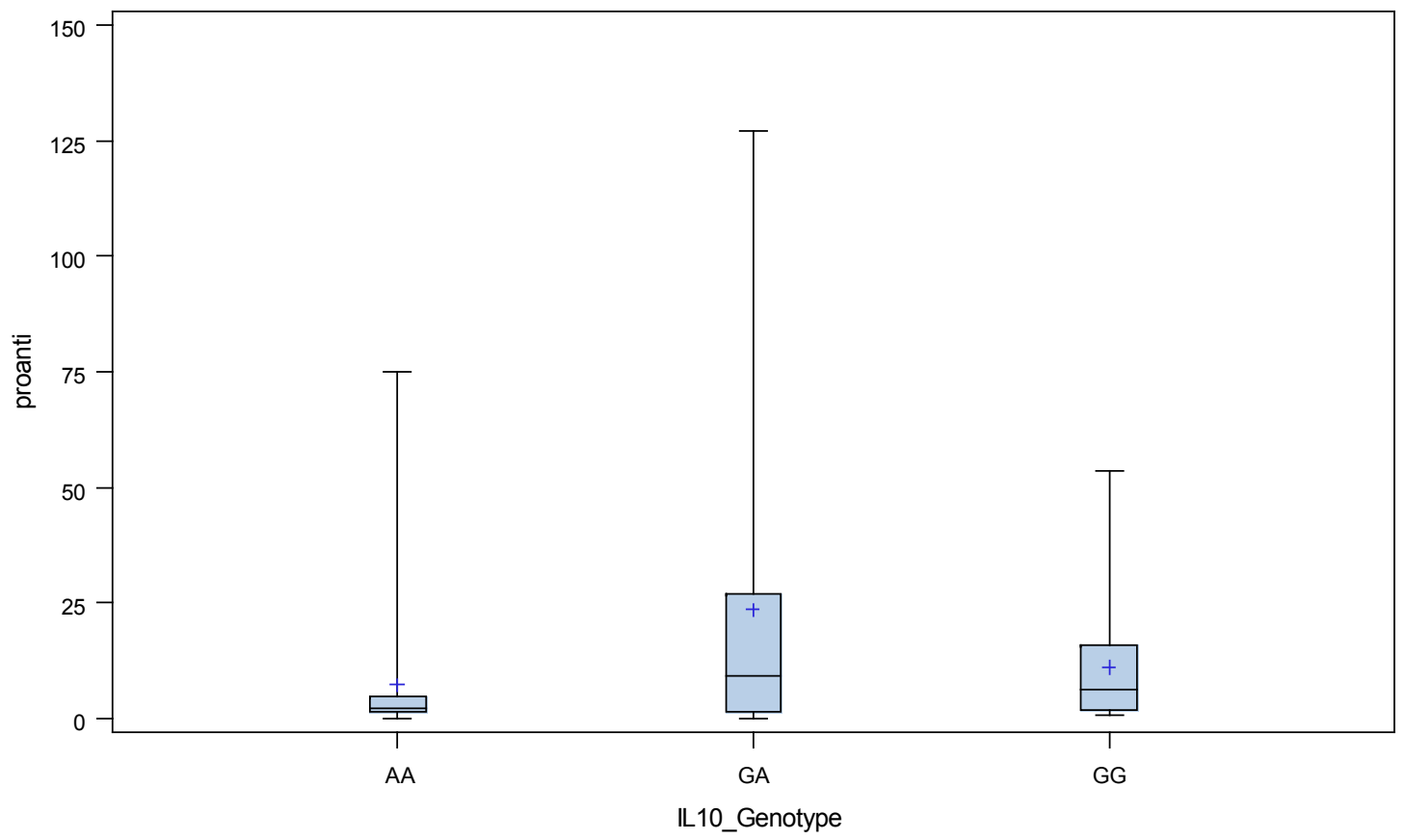

Plasma Log IL-6:IL-10 by IL-10 Genotype

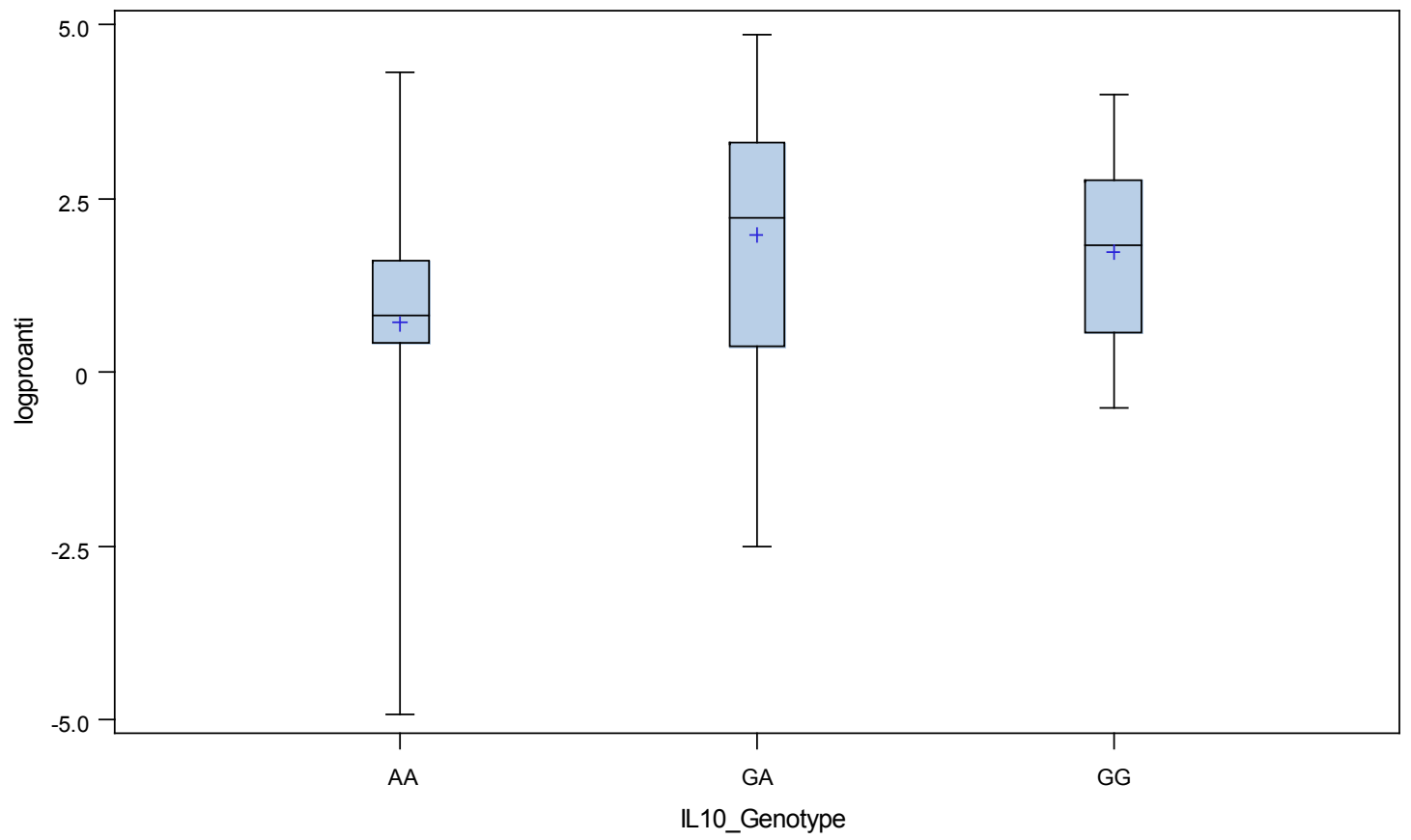

Figure 4-6. Box Plot of Plasma IL-6:IL10 ratios by IL-10 Genotypes Pre and Post Log Transformation. 
ratios among those who did and did not develop subsequent HAI ( $1.8 \pm 1.5$ vs. $1.6 \pm 1.8$, $\mathrm{p}=0.55)$. Cytokine levels were right skewed; thus, non-parametric tests were performed to provide a comparison of median values among participants who did and did not develop HAI. Median cytokine measurements are shown in Table 4-13. There were no significant differences in baseline pro-inflammatory cytokines, anti-inflammatory cytokines, or their ratios among participants who did and did not develop at least one HAI during their ICU stay.

\begin{abstract}
$\operatorname{Aim} 2$
The goal of aim two was to investigate the variance in cytokine genes to determine if they influence levels of protein expression or development of HAI.

The variance in cytokine genes were determined by SNP analysis. The distribution of subjects per genotype is shown in Table 4-14. An ANOVA was performed to examine the difference in cytokine means for each genotype. Table 4-15 and Figure 4-7 summarize the cytokine levels for each SNP. There were no significant differences in plasma IL-6 levels based on SNP rs1800795. Both homozygous AA and GG genotypes for IL-10 SNP rs1800896 were significantly higher (0.02) that the heterozygous GA.

Table 4-14 summarizes development of HAI by genotype and haplotype. There were no statistically significant differences among genotypes or haplotypes for development of HAI. There were no CC_AA, CC_GA, or GG_AA haplotypes among the 17 participants who developed HAI. The most common haplotype, was the heterozygous CG_GA. This haplotype is also where the highest percentage of HAIs occurred but this was not significant.
\end{abstract}

\begin{abstract}
$\operatorname{Aim} 3$
The goal of aim three was to investigate the effects of protein expression levels, genetic variation, and environment on development of HAI. A series of Cox regression analyses were performed among those who did and did not develop HAIs during ICU stay (or up to 28 days in those with a prolonged ICU stay) controlling for a number of potentially confounding variables. Table 4-16 provides a summary of variables testing for inclusion into the multivariate model. Only variables with a p-value of less than 0.25 were included in the multivariate regression model (Table 4-17). There were only four females in the study and it was not appropriate to include gender in the model $(\mathrm{HR}=$ 1313884).

Aim three included a series of questions pertaining to risk of developing HAIs. In general, a hazard ratio greater than 1 indicates a higher risk of developing an event, in this case health care associated infections, and a hazard ratio less than 1 indicates a lower
\end{abstract}


Table 4-13. Comparison of Cytokine Levels among Subjects Developing HAI.

\begin{tabular}{lccc}
\hline \multicolumn{1}{c}{ Variables } & $\begin{array}{c}\text { No HAI } \\
(\mathrm{n}=61)\end{array}$ & $\begin{array}{c}\text { Developed HAI } \\
(\mathrm{n}=17)\end{array}$ & $\begin{array}{c}\mathrm{P} \\
\text { Value }\end{array}$ \\
\hline IL-6 pg/ml & $157.1(251.8)$ & $229.7(571.4)$ & 0.21 \\
IL-10 pg/ml & $18.9(129.6)$ & $34.8(144.6)$ & 0.55 \\
IL-6:IL-10 ratio & $4.7(16.4)$ & $6.2(11.6)$ & 0.78 \\
\hline
\end{tabular}

Note: Median and interquartile range shown. 
Table 4-14. SNP Genotypes and Haplotypes for All Subjects by HAI Development and Pro- and Anti-inflammatory Cytokine Quartiles.

\begin{tabular}{|c|c|c|c|c|c|c|c|c|c|c|}
\hline \multirow[b]{2}{*}{ Variables } & \multirow[b]{2}{*}{$\begin{array}{c}\text { All } \\
\text { Subjects }\end{array}$} & \multirow[b]{2}{*}{ No HAI } & \multirow[b]{2}{*}{ HAI } & \multirow[b]{2}{*}{ P Value } & \multicolumn{3}{|c|}{ Pro-inflammatory Cytokine: IL-6 } & \multicolumn{3}{|c|}{ Anti-inflammatory Cytokine: IL-10 } \\
\hline & & & & & $\begin{array}{c}\text { 1st-3rd } \\
\text { Quartiles }\end{array}$ & $\begin{array}{c}\text { 4th } \\
\text { Quartile }\end{array}$ & $\begin{array}{c}\mathrm{P} \\
\text { Value }\end{array}$ & $\begin{array}{c}\text { 1st-3rd } \\
\text { Quartiles }\end{array}$ & $\begin{array}{c}\text { 4th } \\
\text { Quartile }\end{array}$ & $\begin{array}{c}\mathrm{P} \\
\text { Value }\end{array}$ \\
\hline $\mathrm{N}$ & 78 & 61 & 17 & & 59 & 19 & & 59 & 19 & \\
\hline IL-6 & . & . & . & 0.59 & . & . & 0.67 & . & . & 0.56 \\
\hline $\mathrm{CC}$ & $7(9.0)$ & $6(9.8)$ & $1(5.9)$ & . & $5(8.5)$ & $2(10.5)$ & . & $6(10.1)$ & $1(5.3)$ & . \\
\hline $\mathrm{CG}$ & $28(35.9)$ & $20(32.8)$ & $8(47.1)$ & . & $20(33.9)$ & $8(42.1)$ & . & $19(32.2)$ & $9(47.3)$ & . \\
\hline GG & $43(55.1)$ & $35(57.4)$ & $8(47.1)$ & . & $34(57.6)$ & $9(47.4)$ & . & $34(57.6)$ & $9(47.4)$ & . \\
\hline IL-10 & . & . & . & 0.15 & . & . & 0.26 & . & . & 0.57 \\
\hline AA & $17(21.8)$ & $16(26.2)$ & $1(5.9)$ & . & $15(25.4)$ & $2(10.5)$ & . & $12(20.3)$ & $5(26.3)$ & . \\
\hline GA & $38(48.8)$ & $29(47.5)$ & $9(52.9)$ & . & $29(49.2)$ & $9(47.4)$ & . & $32(54.3)$ & $6(31.6)$ & . \\
\hline GG & $23(29.5)$ & $16(26.2)$ & 7 (41.8) & . & $15(25.4)$ & $8(42.1)$ & . & $15(25.4)$ & $8(42.1)$ & . \\
\hline $\begin{array}{l}\text { Haplotype } \\
\text { (IL-6_IL10) }\end{array}$ & . & & & 0.55 & . & . & 0.47 & . & . & 0.21 \\
\hline CC_AA & $1(1.3)$ & $1(1.6)$ & $0(0)$ & . & $1(1.7)$ & $0(0)$ & . & $0(0)$ & $1(5.3)$ & . \\
\hline CC_GA & $4(5.3)$ & $4(6.6)$ & $0(0)$ & . & $2(3.4)$ & $2(10.5)$ & . & $4(6.8)$ & $0(0)$ & . \\
\hline $\mathrm{CC}^{-} \mathrm{GG}$ & $2(2.3)$ & $1(1.6)$ & $1(5.6)$ & . & $2(3.4)$ & $0(0)$ & . & $2(3.4)$ & $0(0)$ & . \\
\hline CG_AA & $3(3.9)$ & $3(4.9)$ & $0(0)$ & . & $2(3.4)$ & $1(5.3)$ & . & $2(3.4)$ & $1(5.3)$ & . \\
\hline $\mathrm{CG}^{-} \mathrm{GA}$ & $18(23.1)$ & $13(21.3)$ & $5(29.4)$ & . & $14(23.7)$ & $4(21.1)$ & . & $13(22.0)$ & $5(26.3)$ & . \\
\hline $\mathrm{CG}_{-}^{-} \mathrm{GG}$ & $7(9.0)$ & $4(6.6)$ & $3(17.7)$ & . & $4(6.8)$ & $3(15.8)$ & . & $4(6.8)$ & $3(15.8)$ & . \\
\hline $\mathrm{GG}_{-}{ }^{-} \mathrm{AA}$ & $13(16.7)$ & $12(19.7)$ & $1(5.9)$ & . & $12(20.3)$ & $1(5.3)$ & . & $10(17.0)$ & $3(15.8)$ & . \\
\hline GG_GA & $16(20.5)$ & $12(19.7)$ & $4(25.5)$ & . & $13(22.0)$ & $3(15.8)$ & . & $15(25.4)$ & $1(5.3)$ & . \\
\hline GG_GG & $14(18.0)$ & $11(18.0)$ & $3(17.7)$ & . & $9(15.3)$ & $5(26.3)$ & . & $9(15.3)$ & $5(26.3)$ & . \\
\hline IL6 C Allele & 35 (44.9) & $26(42.6)$ & $9(52.9)$ & 0.45 & $25(42.4)$ & $10(52.6)$ & 0.43 & $25(42.4)$ & $10(52.6)$ & 0.43 \\
\hline IL10 A Allele & $55(70.5)$ & $45(73.8)$ & $10(58.8)$ & 0.23 & $44(74.6)$ & $11(57.9)$ & 0.17 & $44(74.6)$ & $11(57.9)$ & 0.17 \\
\hline
\end{tabular}


Table 4-15. Cytokine levels by Genotype.

\begin{tabular}{cccccc}
\hline $\begin{array}{c}\text { Genotype: } \\
\text { IL-6 SNP }\end{array}$ & $\begin{array}{c}\text { Log } \\
\text { IL-6 }\end{array}$ & $\begin{array}{c}\text { Standard } \\
\text { Deviation }\end{array}$ & $\begin{array}{c}\text { Genotype: } \\
\text { IL-10 SNP }\end{array}$ & $\begin{array}{c}\text { Log } \\
\text { IL-10 }\end{array}$ & $\begin{array}{c}\text { Standard } \\
\text { Deviation }\end{array}$ \\
\hline CC & 5.3 & 0.6 & AA & 3.9 & 0.5 \\
CG & 5.1 & 0.3 & GA & 2.6 & 0.3 \\
GG & 4.6 & 0.3 & GG & 3.8 & 0.4 \\
\hline
\end{tabular}




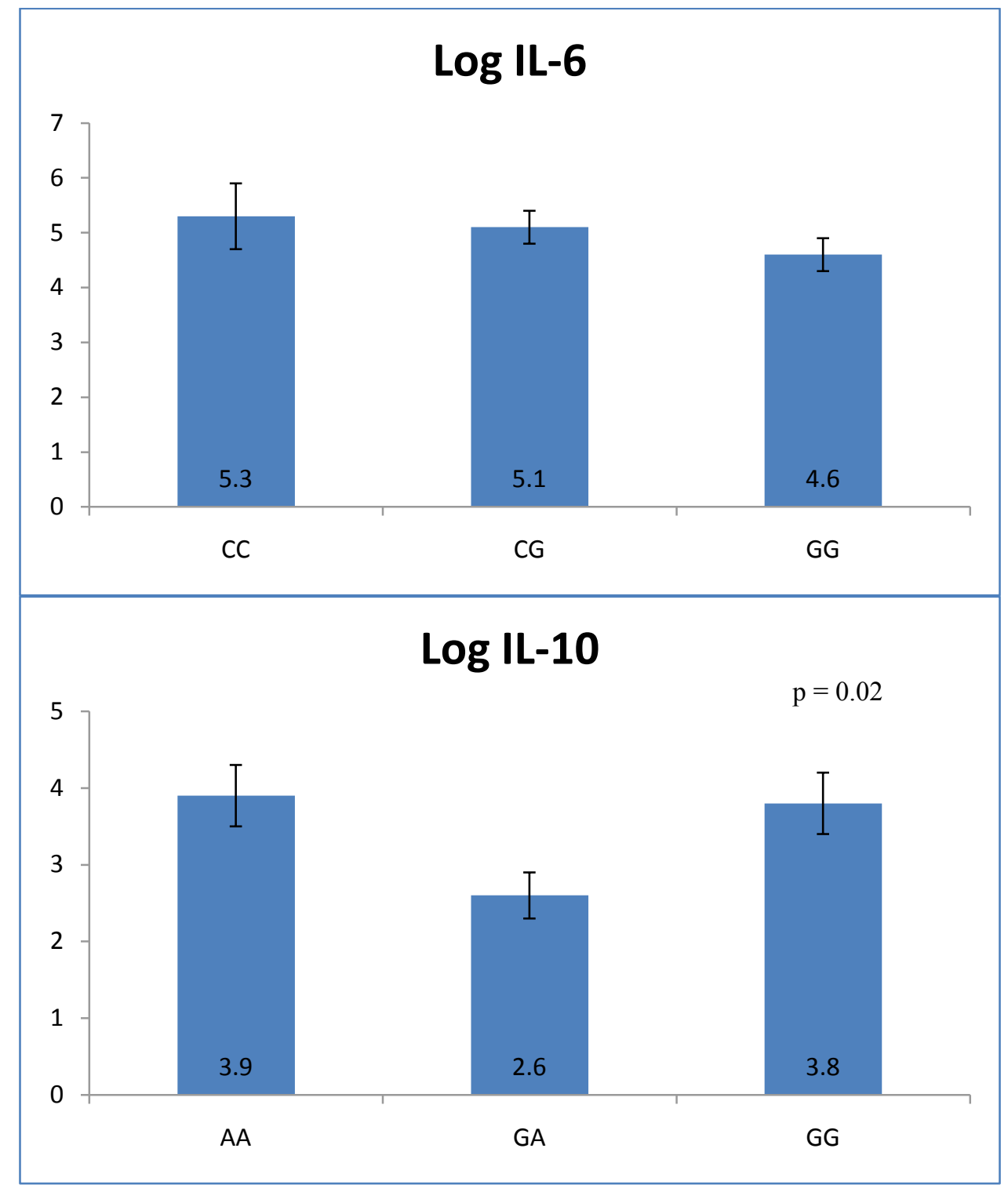

Figure 4-7. Log Plasma Cytokine Levels by Genotype. 
Table 4-16. Univariate Cox Regression Hazard Ratios for Development of HAI.

\begin{tabular}{lccccc}
\hline Variables & $\begin{array}{c}\text { Parameter } \\
\text { Estimate }\end{array}$ & $\begin{array}{c}\text { Standard } \\
\text { Error }\end{array}$ & Chi-Square & $\begin{array}{c}\text { V } \\
\text { Value }\end{array}$ & $\begin{array}{c}\text { Hazard } \\
\text { Ratio }\end{array}$ \\
\hline Age & 0.070 & 0.027 & 6.481 & 0.011 & 1.072 \\
Race & 1.150 & 0.786 & 2.137 & 0.154 & 3.157 \\
Body Mass Index (BMI) & -0.024 & 0.035 & 0.480 & 0.488 & 0.976 \\
Obesity (BMI > 30) & -0.199 & 0.599 & 0.110 & 0.740 & 0.820 \\
Diabetes & -0.638 & 0.595 & 1.152 & 0.283 & 0.528 \\
APACHE II & 0.067 & 0.054 & 1.497 & 0.221 & 1.069 \\
Baseline CRP & 0.002 & 0.002 & 1.695 & 0.193 & 1.002 \\
Baseline Plasma IL-10 & 0.000 & 0.000 & 0.340 & 0.560 & 1.000 \\
Baseline Plasma IL-6 & 0.000 & 0.000 & 0.285 & 0.593 & 1.000 \\
IL-6:IL-10 Ratio & -0.009 & 0.018 & 0.237 & 0.626 & 0.991 \\
IL-6 Genotype: CG & 1.435 & 0.665 & 4.656 & 0.031 & 4.200 \\
IL-6 Genotype: CC & 1.563 & 1.203 & 1.688 & 0.194 & 4.775 \\
IL-10 Genotype: GA & 1.391 & 0.817 & 2.895 & 0.089 & 4.018 \\
IL-10 Genotype: AA & 1.348 & 0.892 & 2.284 & 0.131 & 3.849 \\
Corticosteroids & 0.283 & 0.900 & 0.099 & 0.753 & 1.327 \\
Received Insulin during ICU Stay & -1.184 & 0.805 & 2.163 & 0.141 & 0.306 \\
Any Blood Products during ICU Stay & -0.123 & 0.622 & 0.039 & 0.844 & 0.885 \\
Received Antibiotics Prior to ICU & -0.823 & 0.696 & 1.396 & 0.237 & 0.439 \\
Appropriate Antibiotics in First 24 H & 0.468 & 1.053 & 0.197 & 0.657 & 1.597 \\
Invasive Device Score at HAI & -0.037 & 0.016 & 5.546 & 0.019 & 0.963 \\
Patient: Nurse Ratio > 2 to 1 & 0.494 & 0.616 & 0.644 & 0.422 & 1.639 \\
\hline
\end{tabular}

Note: IL-6 and IL-10 genotype comparisons are made to GG genotype. 
Table 4-17. Final Multivariate Cox Regression Model.

\begin{tabular}{lcccc}
\hline Variables & $\begin{array}{c}\text { Chi- } \\
\text { Square }\end{array}$ & P Value & $\begin{array}{c}\text { Hazard } \\
\text { Ratio }\end{array}$ & $\begin{array}{c}\text { Confidence } \\
\text { Interval }\end{array}$ \\
\hline APACHE II & 5.115 & 0.0237 & 1.241 & $1.029-1.495$ \\
Invasive Device Score at HAI & 11.370 & 0.0007 & 0.908 & $0.086-0.961$ \\
Received Antibiotics Prior to ICU & 4.965 & 0.0259 & 0.106 & $0.015-0.763$ \\
IL-6 Genotype CC & 4.858 & 0.0275 & 28.097 & $1.447-545.653$ \\
IL-6 Genotype CG & 7.456 & 0.0063 & 14.959 & $2.146-104.295$ \\
\hline
\end{tabular}

Note: IL-6 Genotypes are compared to GG genotype. 
risk of developing an event. Each question was answered based on univariate cox regression modeling:

- The hazard ratio to predict development of HAIs for APACHE II is 1.069. For each 10 point increase in APACHE II score the risk ratio or hazard ratio is $\mathrm{e}^{0.06656}$ ${ }^{\mathrm{x} 10}=1.956$. For a 10 point increased in APACHE II score, there is a 95.6 percent increase in the risk of developing a HAI.

- The hazard ratio to predict development of HAI for each additional invasive devise is $\mathrm{e}^{-0.03742}=0.963$. For an additional invasive devise score there was a $3.7 \%$ lower risk of developing a HAI. The cumulative invasive devise score at the time of HAI had been compared to the invasive devise score at ICU discharge among participants who did not develop a HAI.

- The hazard ratio to predict development of HAIs given IL-6 -174G genotype CG compared to GG is $\mathrm{e}^{1.43513}=4.200$, and for IL6 genotype CC compared to GG the hazard ratio is $\mathrm{e}^{1.56334}=4.775$. Presence of the GG genotype has a four-fold increase in risk for development of HAI.

- The hazard ratio to predict development of HAIs given IL-10 -1082G genotypeIL-10 GA compared to GG is $\mathrm{e}^{1.39081}=4.018$, AA compared to GG is $\mathrm{e}^{1.34786}=3.849$. Presence of the GG genotype has a four-fold increase in risk for development of HAI

The hazard ratio to predict development of HAIs for each 10 point increase in pro-inflammatory cytokine based on plasma IL- 6 is $\mathrm{e}^{0.0001308 \times 10}=1.001$. For a 10 point increase in IL- 6 there is a $0.1 \%$ increase in developing a HAI.

- The hazard ratio to predict development of HAIs for each 10 point increase in anti-inflammatory cytokine based on plasma IL-10 is $\mathrm{e}^{0.0002799 \times 10}=1.002$. For a 10 point increase in IL-10 there is a $0.3 \%$ increase in developing a HAI.

- The hazard ratio to predict development of HAIs for each 10 point increase in ratio of pro- to anti-inflammatory cytokine is $\mathrm{e}^{-0.00890 \times 10}=0.915$. For a 10 point increase in IL-6:IL10 ratio there is a 8.5\% lower risk of developing a HAI.

The multivariate model included several predictors associated with HAI development. All variable tested in univariate cox regression with a $\mathrm{p}<0.25$ were included in the final model. These variables included: age, race, APACHE II score, invasive device score at first HAI, received insulin during ICU stay, baseline C-reactive protein, received antibiotics prior to ICU, IL-6 genotype, and IL-10 genotype. Only variables with of probability of $<0.05$ were included in the final model. As some IL-6 genotypes were not significant, a dichotomous variable for genotype was created to compared the $\mathrm{CG}$ and $\mathrm{CC}$ to the GG alleles. The final regression model is shown in Table 4-17. 


\section{CHAPTER 5. DISCUSSION}

The purpose of this study was to determine the impact of baseline systemic inflammation, genetic variability, and environment on the development of HAI among patients with sepsis during their ICU stay. The sample consisted of 78 subjects, with 17 developing at least one HAI during or attributed to their ICU stay. Major findings in general and by aim will be described as well as strengths, limitations, and implications for these findings.

\section{Major Findings}

\section{Baseline Infections}

The most common infection responsible for sepsis at ICU admission in this study was pneumonia, followed by UTI, skin and soft tissue infection, and gastrointestinal or intrabdominal infection. The pattern of infection (site and organism) differs across ICU populations as described in the discussion. This study focuses on medical ICU patients. Our finding of pneumonia as the primary cause of sepsis causing ICU admission is in agreement with some studies ${ }^{43,92}$ but urinary tract infections may precede pneumonia as the primary cause. ${ }^{42}$

We found a significantly higher percentage of patients with high IL-6 levels versus lower levels who developed arrhythmias during their ICU stay $(52.6 \%$ vs. 18.6\%, $p=0.007)$. The median IL-6 difference was 303.3 vs. $98.1(p=0.0008)$ for those who developed arrhythmias versus those who did not. This is consistent with emerging literature. Aviles, et al. have shown that atrial fibrillation is associated with elevated CPR levels, ${ }^{117}$ and Boos provides a summary of four studies that found associations of elevated CRP and IL-6 levels with atrial fibrillation and one study that did not. ${ }^{118}$

This population was composed primarily of males (97.9\%), thus limiting conclusion about females. It is well documented that males have a higher rate of infections; ${ }^{119}$ however; this study included only 4 women. One female $(25 \%)$ and 16 males $(21.6 \%)$ developed HAI. This sample does not include enough females for a valid comparison. There were no differences in the rate of HAI developed between white and black patients in this study; however, we did find a difference in genotype among white

and black patients with sepsis who did and did not develop HAI. The literature reports a higher incidence of infections in blacks as compared with whites. ${ }^{120}$ 


\section{First Health Care Associate Infection}

The percentage of HAI caused by Candida (64\%) was a surprising finding in this study. Typically, the expected leading organisms associated with HAI are gram positive (such as Coagulate-negative Staphylococcus, Staphylococcus aureus, and Enterococcus spp.). ${ }^{43}$ This is a shift away from gram negative organisms in the past, and now Candida species have emerged as the fourth most common cause of health care associated blood stream infections. ${ }^{121,122}$ There were three species of Candida identified (Candida albicans, Candida glabrata, Candida tropicalis) in addition to unidentified Candida species. Colonization of the urinary tract with Candida is becoming more common and the line between colonization and urinary tract infection is not easily distinguished. ${ }^{123}$ Among this sample, there were only two urinary tract infections (Candida species not identified). There were two Candida albicans pneumonias, and all of the remaining Candida infections were bloodstream infections (3 Candida albicans, 2 Candida glabrata, 2 Candida tropicalis). The incidence of fungemia in the ICU had been increasing and there have also been changes in the species pattern of Candida. Candida albicans was the primary species in the 1980s, but now almost half of Candida infections are non-albicans. ${ }^{124}$ The mortality associated with Candida glabrata is higher than Candida albicans, ${ }^{121,124}$ and Candida glabrata has been reported as the second most frequent cause of Candidemia in US hospitals. ${ }^{121}$ Two primary risk factors associated with Candida infections are colonization of the skin and mucous membranes and the presence of invasive devices. ${ }^{122}$ Candida colonization is not always treated and the urinary tract and upper airway may be colonized with Candida. Three patients with Candida infections in this study were not treated. One patient had Candiduria which was not treated and developed Candidemia three days later; however, the blood culture results were not complete until after the patient had expired. Another patient with Candidemia was not treated because care had been withdrawn before results were received, and another patient with Candidemia expired before results were received.

Among all subjects developing infections, less than half (47\%) developed fever or hypothermia, in contrast to $74.4 \%$ experiencing fever or hypothermia at initial presentation. Fever in the ICU is one of the triggers that lead to a careful clinical assessment for its cause and possible laboratory assessments. ${ }^{54}$ Patients receiving corticosteroids have a blunted febrile response due to down-regulation of inflammatory mediators (IL-1 $\beta$, TNF- $\alpha$, IL-6) that cause fever. The threshold for laboratory assessment (blood culture, BAL, and others cultures) is lower in patients receiving corticosteroids, ${ }^{125}$ which may explain why half of the infections were detected in the absence of fever. Meduri et al. identified $60 \%$ of infections occurring after ICU day seven in patients receiving corticosteroids in the absence of fever. ${ }^{126}$ The presentation of HAI required less fluid resuscitation and fewer vasopressors (17\% vs. $32 \%)$, than their initial ICU presentation with sepsis.

A significantly higher number of patients who received corticosteroids developed HAI compared to those who did not $(82.4 \%$ vs. $42.6 \%, \mathrm{p}=0.005)$; however, when controlling for other factors (shown in AIM3) this was not significant. Overall, patients 
who developed HAI had more invasive devices, more days of mechanical ventilation (11.1 vs. $5.9, \mathrm{p}=0.03)$ and more days of ICU stay $(15.3$ vs. $6.4, \mathrm{p}<0.0001)$ when compared to those who did not develop HAI. This is in agreement with literature which consistently shows high correlation of these variables with development of HAI. ${ }^{42}$

\begin{abstract}
Aim 1
The primary goal of aim one was to investigate whether baseline protein expression levels of pro-inflammatory cytokines, anti-inflammatory cytokines, or their ratios influence the development of subsequent HAI in patients with sepsis.

There were no significant differences in levels of pro-inflammatory cytokine, anti-inflammatory cytokine, or their ratio among subjects who did and did not develop at least one HAI during their ICU stay. Specifically, an exaggerated pro-inflammatory response was present in $6(31.6 \%)$ compared to $11(18.6 \%)$ participants without an exaggerated pro-inflammatory response who developed subsequent HAI. This difference was not significant $(\mathrm{p}=0.55)$. Likewise, an exaggerated anti-inflammatory response was present in $5(26.3 \%)$ compared to $12(20.3)$ participants without an exaggerated antiinflammatory response who developed subsequent HAI. This difference was also not significant $(\mathrm{p}=0.43)$. There was also no significant difference in the log of proinflammatory to anti-inflammatory ratios among those who did and did not develop subsequent HAI.
\end{abstract}

One study investigated a similar relationship. Ramirez et al. performed a prospective observational study to examine the relationship between systemic inflammatory response and development of ventilator-associated pneumonia (VAP). ${ }^{127}$ They included patients on mechanical ventilation who were expected to remain on mechanical ventilation for more than 48 hours. They excluded patients who developed other HAIs. They measured several cytokine (IL-6, Il-10, and others) at baseline and then every 96 hours. Their sample included 44 patients, among which 9 developed VAP. Findings included higher baseline IL-6 pg/ml among patients who subsequently developed VAP. Higher median IL-6 pg/ml were reported for confirmed VAP cases (235 (188-620)) as compared to non-suspected (92 (43 - 167)), suspected (120 (112 - 161)) VAP cases $(\mathrm{p}=0.02)$. They found no significant difference in median IL-10 pg/ml for non-suspected $(0(0-4))$, suspected $(0(0-4))$, and confirmed $(6(3-12))$ cases of VAP $(\mathrm{p}=0.16)$. They reported IL-10 detection limits of $1 \mathrm{pg} / \mathrm{ml}$.

The findings of Ramirez provide limited evidence that a clinical relationship between exaggerated pro-inflammatory response and development of infections exists. ${ }^{127}$ In our study, we were unable to identify a difference. Our findings may be limited by the timing of cytokine measurement and other factors. Baseline cytokine measurements were collected at enrollment within 72 hours of developing sepsis. IL-6 levels usually peak after IL- $1 \beta$ and TNF- $\alpha$, around eight hours following an insult. The variation in timing 
for each subject $(0-72$ hours $)$ and use of corticosteroids $(<1 \mathrm{mg} / \mathrm{kg})$ may have limited these findings.

\begin{abstract}
$\operatorname{Aim} 2$
The goal of aim two was to investigate the variance in cytokine genes to determine if they influence levels of protein expression or development of HAI.
\end{abstract}

\title{
Genotye and Cytokine Level
}

We examined cytokine levels by genotype and compared subjects grouped by degree of inflammatory response. In Chi-square analysis comparing subjects with an exaggerated inflammatory response to those without an exaggerated response, we found no difference for rs 1800795 or rs 1800896 . There were no significant differences in median or log IL-6 level based on genotype rs1800795. We did find a significant difference in IL-10 level based on genotype rs1800896. Participants who were homozygous AA or GG had significantly higher log IL-10 levels than heterozygous GA genotypes (0.02).

While promoter polymorphism may increase or decrease transcription levels, there are also epigenetic and other factors that influence gene regulation. Taudorf et al. studied the cytokine response to an endotoxin challenge in 200 young healthy men. They found up to a 6 fold increase in cytokine levels following endotoxin injection but they did not find significant differences in cytokine levels among many commonly studies cytokine SNPs, including rs1800896 and rs $1800795 .{ }^{100}$ Among all comparisons involving genotype and cytokine level, we only found one difference. Other studies have shown varied results.

Our findings, or lack of findings, may be limited by several factors. First, as noted in aim one, timing of cytokine measurement may be a factor. An underlying assumption of this research was that cytokine levels would be persistently elevated. This has been well documented in the literature, particularly for non-survivors. ${ }^{87-89}$ This assumption could not be evaluated in this study because plasma samples were only collected at baseline and not over time. Second, variability in cytokine measures may be a factor. This variability ranges from technician technique to the method selected for cytokine measurement to the various proprietary antibodies used by different biotech companies for each cytokine. Lastly, the findings of Taudorf suggest that either there may be no difference or, more likely, there are several other factors that may be involved such that these differences are not expressed in a healthier or less acutely ill population. 


\section{Genotype and Development of HAI}

There were no statistically significant differences among genotypes or haplotypes for development of HAI. There were no CC_AA, CC_GA, or GG_AA haplotypes among the 17 participants who developed HAI. The CC_GG haplotype was not observed in another study. ${ }^{128}$ The most common haplotype was the heterozygous CG_GA. Baier, et al., in a retrospective review of 293 low birth weight infants, investigated the IL-6 rs1800795 and IL-10 rs1800896 SNPs and sepsis outcomes. In their study, the rs 1800795 C Allele was associated with late blood stream infections in African American but not Caucasian infants. The incidence of blood stream infections was $69 \%$ in African American infants with the C Allele compared to $46 \%$ in African American infants with the GG genotype $(p=0.02)$. Racial differences have not been investigated in this dissertation study and will be explored in future analyses. Baier et al. also found an increased association of the rs 1800896 A Allele with increased incidence of late blood stream infections. ${ }^{128}$ The rs 1800795 CC genotype has been associated with fungal blood stream infections in Caucasians. This study population includes a large number of fungal blood stream infections. Among the 7 participants with the rs1800795 CC genotype only one developed a HAI, which was fungemia caused by Candida albicans.

\section{$\operatorname{Aim} 3$}

The goal of aim three was to investigate the effects of protein expression levels, genetic variation, and environment on development of HAI. A series of Cox regression analyses were performed among those who did and did not develop HAIs during ICU stay. Although the univariate models provided important risk ratios, the multivariate model provides the variables most predictive for the risk ratio and can be used to predict the risk of HAI development. We found no difference in protein expression levels, one genetic variation, and two environmental factors that strongly predict risk of HAI development. The final model included APACHE II, invasive devise score, and antibiotics prior to ICU, and IL6 rs 1800795.

Severity of illness contributes to risk of HAI development. Based on the multivariate model, every one point increase in APACHE II score there is a $3.4 \%$ increase in the risk of developing HAI. APACHE II scores range from 0 to 71 with a higher scores associated with a worse outcome. ${ }^{38}$ The APACHE II has been correlated with baseline inflammatory response but not HAI development. ${ }^{12}$ Patients with a higher severity of illness require a higher level of care. These patients are potentially exposed to a higher risk due to the presence of more invasive devices and also have a higher exposure to multiple hospital personnel and equipment needed to provide their care. For each additional invasive device there was a 9.1\% reduced risk in the development of HAI. This does not make intuitive sense. It is likely that the format of the invasive device score used in the model is problematic and additional models will be explored using only the number of invasive devices present at the time of HAI, rather than the cumulative score. Table 4-6 provides the cumulative invasive device score at the time of HAI (52.8 
$\pm 26.7)$ and Table 4-8 provides the cumulative invasive device score at ICU discharge (23.9 \pm 23.0$)$ for those who did not develop HAI. More investigation is needed to fully assess these findings.

We found that early antibiotic use prior to ICU stay reduces risk of HAI in patients with sepsis. The importance of early antibiotic use has been incorporated into several guidelines. The surviving sepsis campaign recommends antibiotics within the first hour for septic shock. ${ }^{26}$ The IDSA recommends antibiotic administration in the ER prior to ICU admission for patients with CAP. ${ }^{55}$ In this study, we examined whether patients received antibiotics before transfer to the ICU. The early administration of antibiotics reduces morality associated with sepsis and may, according to Zubert, be a "surrogate marker for quality of care in the broader sense". ${ }^{129}$ While early antibiotic use is important, appropriately deescalating therapy based on culture sensitivities is also important. ${ }^{130}$

The presence of IL6 rs 1800795 CC compared to GG and GC compared to GG had a higher risk in the univariate mode, and the risk increased in the multivariate model when controlling for other variables. The final model included only variables that were significant $(\mathrm{p}<0.05)$. The higher risk associated with rs 1800795 genotypes $\mathrm{CG}$ and CC is consistent with higher risk associated with the $\mathrm{C}$ allele noted in the literature. The rs 1800795 C Allele has also been found to be associated with late blood stream infections in African American infants, ${ }^{128}$ and has been shown to be more prevalent in coronary artery patients developing myocardial infarction compared to coronary artery patients with stable and unstable angina. ${ }^{131}$ More analysis is needed to fully explore the relationships of these variables.

\section{Strengths}

Study strengths are summarized below:

1. This study occurred in a teaching hospital where the attending physicians and medical team rotate monthly; however, the MICU team include a PharmD who is present for daily rounds with the team in the morning and afternoon on Monday through Friday. This provides consistency in patient management in regards to the appropriate use, selection, and dosing of antibiotics in the ICU. This PharmD reviewed cultures and antibiotics with me to determine appropriate antibiotics based on current guidelines.

2. This study occurred in one facility, limiting the biases that could occur at multiple sites by multiple data collectors.

3. The inclusion of biomarkers and use in a multivariate model strengthens conclusions about risk factors associated with development of HAI. 


\section{Limitations}

Study limitations are summarized below:

1. A potential limitation of this study was the heterogeneous sample. Some patients were not as sick as others and were discharged from the ICU prior to three days. Since development of HAI has been strongly associated with length of stay, this is a limitation that was accounted for by using Cox regression modeling.

2. Limiting this study to a single site with primarily older male veterans limits generalizability of findings.

3. It was a major assumption that baseline systemic inflammation would be prolonged. Measuring only baseline cytokine levels is a limitation.

4. The usage of corticosteroid therapy may impact the degree and duration of systemic inflammation; thus, potentially limiting the possible impact of systemic inflammation on the development of HAI in participants receiving corticosteroids.

5. The use of corticosteroids may limit fever among participants experiencing HAI, and may result in failure to detect HAI when they occur. It is recommended clinical practice in our ICU to use sepsis surveillance, and thus a high degree of suspicion when steroids are used.

6. There may be other predisposing factors for development of HAI that were not measured.

7. The investigator is a novice bench researcher, and although efforts were made to accurately follow protocols, it is possible that errors could have influenced results.

8. Endpoint genotyping of rs1800896 required manual calls in seven samples.

9. Interleukin 6 was selected as a proinflammatory cytokine; however, it does have some anti-inflammatory properties.

\section{Implications}

This study provides important insights into risk factors that contribute to the development of HAI in patients presenting to the ICU with sepsis. These findings may impact nursing and other critical care clinician practice first by helping to identify patients at risk, then implementing stricter targeted infection control practices in efforts to prevent development of HAIs (in addition to current standard and recommended practices). JACHO patient safety goals include prevention of HAI.

Since the completion of this study, new processes are in place in the facility where this study occurred. These including daily surveillance of central line sites and implementation of a UTI bundle to reduce UTI. The findings of this study reveal a need to further investigate the cause of Candida in this population and to follow-up on the incidence of Candida in this unit. The use of antibiotic timing and duration should be reviewed. A high percentage ( 9 of $11,81.8 \%$ ) of the Candida infections occurred during corticosteroids use, indicating a need to assure appropriate sepsis surveillance is followed in patients receiving corticosteroids. Specific nursing measures would include a review of 
standard IV care practices such as tubing changes, site rotation for peripheral lines, duration of central lines, routine site evaluation and care, hub care, cleaning of IV equipment, cleaning of transducer holders, and no re-use of disposable pressure bags. This may include changing the catheter hub after blood draws when flushing cannot completely clear the hub as well as protocol driven hub care.

Cleaning the environment closest to the patient needs to be considered. The side rails, call light, bed controls, and equipment in use in the room are typically only cleaned when they become soiled. These items could be wiped down with sanitary wipes daily when other areas of the room are cleaned. Cleaning of other environmental areas would include routine cleaning of medication carts, including the front of medication drawers, keyboard, scanner, and the work surface. Routine cleaning of the sink handles and light switches should also be evaluated.

\section{Conclusions}

This study provides evidence of a genetic risk for development of HAI. Despite best evidenced based practices some patients will develop HAI. Strict aseptic technique is essential to preventing infection. In addition to eliminating invasive devices as quickly as possible, patients with a high severity of illness may need to be isolated to lower their risk. Early administration of antibiotics not only provides prompt treatment for the initial infection but also lowers risk for subsequent infections. 


\section{LIST OF REFERENCES}

1. Ylipalosaari P, Ala-Kokko TI, Laurila J, Ohtonen P, Syrjala H. Epidemiology of intensive care unit (ICU)-acquired infections in a 14-month prospective cohort study in a single mixed Scandinavian university hospital ICU. Acta Anaesthesiologica Scandinavica 2006;50:1192-7.

2. Ylipalosaari P, Ala-Kokko TI, Laurila J, Ohtonen P, Syrjala H. Intensive care acquired infection is an independent risk factor for hospital mortality: a prospective cohort study. Critical Care 2006;10:R66.

3. Munford RS. Sepsis and Septic Shock. In: Fauci AS, Braunwald E, Isselbacher KJ, et al., eds. Harrison's Principles of Internal Medicine. 14 ed. New York, NY: McGraw-Hill Health Professions Division; 1998:776-80.

4. Tolley E. Causal Modeling of Infection in the Activated Host: Implications for Clinical Investigaton in Sepsis and Acute Respiratory Distress Syndrome. Sepsis 2000;4:21-33.

5. Osmon S, Warren D, Seiler SM, Shannon W, Fraser VJ, Kollef MH. The influence of infection on hospital mortality for patients requiring $>48 \mathrm{~h}$ of intensive care. Chest 2003;124:1021-9.

6. Darouiche RO. Device-associated infections: a macroproblem that starts with microadherence. Clinical Infectious Diseases 2001;33:1567-72.

7. Richards MJ, Edwards JR, Culver DH, Gaynes RP. Nosocomial infections in combined medical-surgical intensive care units in the United States. Infection Control Hospital Epidemiology 2000;21:510-5.

8. Rosenthal VD, Maki DG, Salomao R, et al. Device-associated nosocomial infections in 55 intensive care units of 8 developing countries. Annals of Internal Medicine 2006;145:582-91.

9. McCusker ME, Perisse AR, Roghmann MC. Severity-of-illness markers as predictors of nosocomial infection in adult intensive care unit patients. American Journal of Infection Control 2002;30:139-44.

10. Fernandez-Crehuet R, Diaz-Molina C, de Irala J, Martinez-Concha D, SalcedoLeal I, Masa-Calles J. Nosocomial infection in an intensive-care unit: identification of risk factors. Infection Control Hospital Epidemiology 1997;18:825-30. 
11. Gogos CA, Drosou E, Bassaris HP, Skoutelis A. Pro- versus anti-inflammatory cytokine profile in patients with severe sepsis: a marker for prognosis and future therapeutic options. Journal Infectious Diseases 2000;181:176-80.

12. Kinasewitz GT, Yan SB, Basson B, et al. Universal changes in biomarkers of coagulation and inflammation occur in patients with severe sepsis, regardless of causative micro-organism [ISRCTN74215569]. Critical Care (London, England) 2004;8:R82-90.

13. Villar J, Maca-Meyer N, Perez-Mendez L, Flores C. Bench-to-bedside review: understanding genetic predisposition to sepsis. Critical Care (London, England) $2004 ; 8: 180-9$.

14. Holmes CL, Russell JA, Walley KR. Genetic polymorphisms in sepsis and septic shock: role in prognosis and potential for therapy. Chest 2003;124:1103-15.

15. Stuber F, Klaschik S, Lehmann LE, Schewe JC, Weber S, Book M. Cytokine promoter polymorphisms in severe sepsis. Clinical Infectious Diseases 2005;41 Suppl 7:S416-20.

16. Dahmer MK, Randolph A, Vitali S, Quasney MW. Genetic polymorphisms in sepsis. Pediatric Critical Care Medicine 2005;6:S61-73.

17. Wurfel MM, Gordon AC, Holden TD, et al. Toll-like receptor 1 polymorphisms affect innate immune responses and outcomes in sepsis. American Journal of Respiratory and Critical Care Medicine 2008.

18. Arcaroli J, Fessler MB, Abraham E. Genetic polymorphisms and sepsis. Shock (Augusta, Ga) 2005;24:300-12.

19. Papathanassoglou ED, Giannakopoulou MD, Bozas E. Genomic variations and susceptibility to sepsis. AACN Advanced Critical Care 2006;17:394-422.

20. Segal S, Hill AV. Genetic susceptibility to infectious disease. Trends in Microbiology 2003;11:445-8.

21. Burgner D, Jamieson SE, Blackwell JM. Genetic susceptibility to infectious diseases: big is beautiful, but will bigger be even better? The Lancet Infectious Diseases 2006;6:653-63.

22. van der Poll T, Opal SM. Host-pathogen interactions in sepsis. The Lancet Infectious Diseases 2008;8:32-43. 
23. Angus DC, Linde-Zwirble WT, Lidicker J, Clermont G, Carcillo J, Pinsky MR. Epidemiology of severe sepsis in the United States: analysis of incidence, outcome, and associated costs of care. Critical Care Medicine 2001;29:1303-10.

24. Umberger R, Thompson C, Muthiah M, Meduri GU. Patients admitted to ICU with sepsis are more likely to develop nosocomial infections. Chest 2008;134(suppl) e479.

25. Janeway CA, Travers P, Walport M, Shlomchik MJ. Immunobiology - the immune system in health and disease. 6 ed. New York, NY: Garland Science Publishing; 2005.

26. Dellinger RP, Carlet JM, Masur H, et al. Surviving sepsis campaign guidelines for management of severe sepsis and septic shock. Critical Care Medicine 2004;32:858-73.

27. Otero RM, Nguyen HB, Huang DT, et al. Early goal-directed therapy in severe sepsis and septic shock revisited: concepts, controversies, and contemporary findings. Chest 2006;130:1579-95.

28. Rivers E, Nguyen B, Havstad S, et al. Early goal-directed therapy in the treatment of severe sepsis and septic shock. The New England Journal of Medicine 2001;345:1368-77.

29. Bone RC, Balk RA, Cerra FB, et al. Definitions for sepsis and organ failure and guidelines for the use of innovative therapies in sepsis. The ACCP/SCCM Consensus Conference Committee. American College of Chest Physicians/Society of Critical Care Medicine. Chest 1992;101:1644-55.

30. Levy MM, Fink MP, Marshall JC, et al. 2001 SCCM/ESICM/ACCP/ATS/SIS international sepsis definitions conference. Critical Care Medicine 2003;31:12506.

31. Angus DC, Burgner D, Wunderink R, et al. The PIRO concept: $\mathrm{P}$ is for predisposition. Critical Care (London, England) 2003;7:248-51.

32. Gerlach H, Dhainaut JF, Harbarth S, Reinhart K, Marshall JC, Levy M. The PIRO concept: $\mathrm{R}$ is for response. Critical Care (London, England) 2003;7:256-9.

33. Vincent JL, Opal S, Torres A, Bonten M, Cohen J, Wunderink R. The PIRO concept: I is for infection. Critical Care (London, England) 2003;7:252-5.

34. Vincent JL, Wendon J, Groeneveld J, Marshall JC, Streat S, Carlet J. The PIRO concept: O is for organ dysfunction. Critical Care (London, England) 2003;7:2604. 
35. Opal SM. Concept of PIRO as a new conceptual framework to understand sepsis. Pediatric Critical Care Medicine 2005;6:S55-60.

36. Vincent JL, de Mendonca A, Cantraine F, et al. Use of the SOFA score to assess the incidence of organ dysfunction/failure in intensive care units: results of a multicenter, prospective study. Working group on "sepsis-related problems" of the European Society of Intensive Care Medicine. Critical Care Medicine 1998;26:1793-800.

37. Bernard GR, Doig G, Hudson LD, et al. Quantification of organ failure for clinical trials and clinical practice. American Journal of Respiratory and Critical Care Medicine 1993;151:A323.

38. Knaus WA, Draper EA, Wagner DP, Zimmerman JE. APACHE II: a severity of disease classification system. Critical Care Medicine 1985;13:818-29.

39. Pagon RA, Bird TD, Dolan CR, Stephens K. GeneReviews. In. Seattle (WA): University of Washington, Seattle; 1993-2011.

40. Eggimann P, Pittet D. Infection control in the ICU. Chest 2001;120:2059-93.

41. Mims C, Dockrell HM, Goering RV, Roitt I, Wakelin D, Zuckerman M. Medical microbiology. 3 ed. Philadelphia, PA: Elsevier Mosby; 2004.

42. Richards MJ, Edwards JR, Culver DH, Gaynes RP. Nosocomial infections in medical intensive care units in the United States. National Nosocomial Infections Surveillance System. Critical Care Medicine 1999;27:887-92.

43. Vincent JL, Bihari DJ, Suter PM, et al. The prevalence of nosocomial infection in intensive care units in Europe. Results of the European prevalence of infection in intensive care (EPIC) study. EPIC International Advisory Committee. Journal of the American Medical Association1995;274:639-44.

44. Fridkin SK, Gaynes RP. Antimicrobial resistance in intensive care units. Clinics in Chest Medicine 1999;20:303-16, viii.

45. Bryant S, Wilbeck J. Vancomycin-resistant enterococcus in critical care areas. Critical Care Nursing Clinics of North America 2007;1:69-75.

46. Dunlap JT. Methicillin-Resistant Staphylococcus aureus in critical care areas. Critical Care Nursing Clinics of North America 2007;1:61-8.

47. Smith MA, McInnis LA. Antimicrobial resistance in critical care. Critical Care Nursing Clinics of North America 2007;1:53-60. 
48. Bennett KM, Scarborough JE, Sharpe M, et al. Implementation of antibiotic rotation protocol improves antibiotic susceptibility profile in a surgical intensive care unit. The Journal of Trauma 2007;63:307-11.

49. Warren DK, Hill HA, Merz LR, et al. Cycling empirical antimicrobial agents to prevent emergence of antimicrobial-resistant gram-negative bacteria among intensive care unit patients. Critical Care Medicine 2004;32:2450-6.

50. McGowan JE, Jr. Minimizing antimicrobial resistance in hospital bacteria: can switching or cycling drugs help? Infection Control 1986;7:573-6.

51. Polk RE. Antimicrobial formularies: can they minimize antimicrobial resistance? American Journal of Health Systems Pharmacy 2003;60:S16-9.

52. El-Azizi M, Mushtaq A, Drake C, et al. Evaluating antibiograms to monitor drug resistance. Emerging Infectious Diseases 2005;11:1301-2.

53. O'Grady NP, Alexander M, Dellinger EP, et al. Guidelines for the prevention of intravascular catheter-related infections. Centers for Disease Control and Prevention. MMWR - Recommendations and Reports 2002;51:1-29.

54. O'Grady NP, Barie PS, Bartlett JG, et al. Practice guidelines for evaluating new fever in critically ill adult patients. Task Force of the Society of Critical Care Medicine and the Infectious Diseases Society of America. Clinical Infectious Diseases 1998;26:1042-59.

55. Mandell LA, Wunderink RG, Anzueto A, et al. Infectious Diseases Society of America/American Thoracic Society consensus guidelines on the management of community-acquired pneumonia in adults. Clinical Infectious Diseases 2007;44 Suppl 2:S27-72.

56. Craven DE. Preventing ventilator-associated pneumonia in adults: sowing seeds of change. Chest 2006;130:251-60.

57. Dellit TH, Owens RC, McGowan JE, Jr., et al. Infectious Diseases Society of America and the Society for Healthcare Epidemiology of America guidelines for developing an institutional program to enhance antimicrobial stewardship. Clinical Infectious Diseases 2007;44:159-77.

58. Boyce JM, Pittet D. Guideline for Hand Hygiene in Health-care settings. recommendations of the healthcare infection control practices advisory committee and the HICPAC/SHEA/APIC/IDSA hand hygiene task force. Society for Healthcare Epidemiology of America/Association for Professionals in Infection Control/Infectious Diseases Society of America. MMWR - Recommendations and Reports 2002;51:1-45, quiz CE1-4. 
59. Siegel JD, Rhinehart E, Jackson M, Chiarello L. 2007 Guideline for isolation precautions: preventing transmission of infectious agents in health care settings. American Journal of Infection Control 2007;35:S65-164.

60. Rutala WA, Weber DJ. Disinfection and sterilization in health care facilities: what clinicians need to know. Clinical Infectious Diseases 2004;39:702-9.

61. Girou E, Stephan F, Novara A, Safar M, Fagon JY. Risk factors and outcome of nosocomial infections: results of a matched case-control study of ICU patients. American Journal of Respiratory and Critical Care Medicine 1998;157:1151-8.

62. Moreno CA, Rosenthal VD, Olarte N, et al. Device-associated infection rate and mortality in intensive care units of 9 Colombian hospitals: findings of the International Nosocomial Infection Control Consortium. Infection Control and Hospital Epidemiology 2006;27:349-56.

63. Lorente L, Villegas J, Martin MM, Jimenez A, Mora ML. Catheter-related infection in critically ill patients. Intensive Care Medicine 2004;30:1681-4.

64. Maki DG, Kluger DM, Crnich CJ. The risk of bloodstream infection in adults with different intravascular devices: a systematic review of 200 published prospective studies. Mayo Clinic Proceedings 2006;81:1159-71.

65. Schierholz JM, Lucas LJ, Rump A, Pulverer G. Efficacy of silver-coated medical devices. Journal of Hospital Infections 1998;40:257-62.

66. Hanna HA, Raad, II, Hackett B, et al. Antibiotic-impregnated catheters associated with significant decrease in nosocomial and multidrug-resistant bacteremias in critically ill patients. Chest 2003;124:1030-8.

67. Wolcott R, Dowd S. The role of biofilms: are we hitting the right target? Plastic and Reconstructive Surgery 2011;127 Suppl 1:28S-35S.

68. Asad S, Opal SM. Bench-to-bedside review: Quorum sensing and the role of cellto-cell communication during invasive bacterial infection. Critical Care (London, England) 2008;12:236.

69. O'Gara JP, Humphreys H. Staphylococcus epidermidis biofilms: importance and implications. Journal of Medical Microbiology 2001;50:582-7.

70. Allaker RP. The use of nanoparticles to control oral biofilm formation. Journal of Dental Research 2010;89:1175-86. 
71. Urden L, Stacy K, Lough M. Systemic inflammatory response syndrome and multiple organ dysfunction syndrome. In: Bruno V, ed. Thelan's Critical Care Nursing Diagnosis and Management. St. Louis, MO: Mosby; 2006:1032-46.

72. Kumar V, Abbas A, Fausto N. Acute and chronic inflammation. In: Gruliow R, ed. Robbin and Contan Pathologic Basis of Disease. 7th ed. Philadelphia, PA: Elsevier Inc.; 2005:47-86.

73. Abbas A. Diseases of Immunity. In: Gruliow R, ed. Robbin and Contan Pathologic Basis of Disease. 7th ed. Philadelphia, PA: Elsevier Inc.; 2005:193267.

74. Wilson CB. Host defense mechanisms: the cellular immune system and its role in host defense. In: Mandell GL, Douglas RG, Jr., Bennett JE, eds. Principles and Practices of Infectious Diseases. New York, NY: Churchill Livingston Inc.; 1990:101-38.

75. Opal SM, DePalo VA. Anti-inflammatory cytokines. Chest 2000;117:1162-72.

76. Kanangat S, Bronze MS, Meduri GU, et al. Enhanced extracellular growth of Staphylococcus aureus in the presence of selected linear peptide fragments of human interleukin (IL)-1 beta and IL-1 receptor antagonist. The Journal of Infectious Diseases 2001;183:65-9.

77. Kanangat S, Meduri GU, Tolley EA, et al. Effects of cytokines and endotoxin on the intracellular growth of bacteria. Infection and Immunity 1999;67:2834-40.

78. Meduri GU, Kanangat S, Bronze M, et al. Effects of methylprednisolone on intracellular bacterial growth. Clinical and Diagnostic Laboratory Immunology 2001;8:1156-63.

79. Meduri GU, Kanangat S, Stefan J, Tolley E, Schaberg D. Cytokines IL-1beta, IL6 , and TNF-alpha enhance in vitro growth of bacteria. American Journal of Respiratory and Critical Care Medicine 1999;160:961-7.

80. Ashare A, Powers LS, Butler NS, Doerschug KC, Monick MM, Hunninghake GW. Anti-inflammatory response is associated with mortality and severity of infection in sepsis. American Journal of Physiology 2005;288:L633-40.

81. Dinarello CA. Proinflammatory cytokines. Chest 2000;118:503-8.

82. Alberts B, Bray D, Lewis J, Raff M, Roberts K, Watson J, eds. Molecular biology of the cell. 3 ed. New York, NY: Garlin Publishing, Inc.; 1994. 
83. Darville T, O'Neill JM, Andrews CW, Jr., Nagarajan UM, Stahl L, Ojcius DM. Toll-like receptor-2, but not toll-like receptor-4, is essential for development of oviduct pathology in chlamydial genital tract infection. Journal of Immunology 2003;171:6187-97.

84. Stephan F, Yang K, Tankovic J, et al. Impairment of polymorphonuclear neutrophil functions precedes nosocomial infections in critically ill patients. Critical Care Medicine 2002;30:315-22.

85. Krutzik SR, Sieling PA, Modlin RL. The role of toll-like receptors in host defense against microbial infection. Current Opinion in Immunology 2001;13:104-8.

86. Kobayashi KS, Flavell RA. Shielding the double-edged sword: negative regulation of the innate immune system. Journal of Leukocyte Biology 2004;75:428-33.

87. Headley AS, Tolley E, Meduri GU. Infections and the inflammatory response in acute respiratory distress syndrome. Chest 1997;111:1306-21.

88. Meduri GU, Headley S, Kohler G, et al. Persistent elevation of inflammatory cytokines predicts a poor outcome in ARDS. Plasma IL-1 beta and IL-6 levels are consistent and efficient predictors of outcome over time. Chest 1995;107:106273.

89. Kellum JA, Kong L, Fink MP, et al. Understanding the inflammatory cytokine response in pneumonia and sepsis: results of the genetic and inflammatory markers of sepsis (GenIMS) study. Archives of Internal Medicine 2007;167:165563.

90. Hoover L, Bochicchio GV, Napolitano LM, et al. Systemic inflammatory response syndrome and nosocomial infection in trauma. The Journal of Trauma 2006;61:310-6; discussion 6-7.

91. Bochicchio GV, Napolitano LM, Joshi M, et al. Persistent systemic inflammatory response syndrome is predictive of nosocomial infection in trauma. The Journal of Trauma 2002;53:245-50; discussion 50-1.

92. Bernard GR, Vincent JL, Laterre PF, et al. Efficacy and safety of recombinant human activated protein $\mathrm{C}$ for severe sepsis. The New England Journal of Medicine 2001;344:699-709.

93. Ulloa L, Tracey KJ. The "cytokine profile": a code for sepsis. Trends in Molecular Medicine 2005;11:56-63. 
94. Taniguchi T, Koido Y, Aiboshi J, Yamashita T, Suzaki S, Kurokawa A. Change in the ratio of interleukin- 6 to interleukin-10 predicts a poor outcome in patients with systemic inflammatory response syndrome. Critical Care Medicine 1999;27:1262-4.

95. Remick DG, Bolgos G, Copeland S, Siddiqui J. Role of interleukin-6 in mortality from and physiologic response to sepsis. Infection and Immunity 2005;73:2751-7.

96. Jones SA. Directing transition from innate to acquired immunity: defining a role for IL-6. Journal of Immunology 2005;175:3463-8.

97. Dinarello CA. Proinflammatory and anti-inflammatory cytokines as mediators in the pathogenesis of septic shock. Chest 1997;112:321S-9S.

98. Yende S, Kammerer CM, Angus DC. Genetics and proteomics: deciphering gene association studies in critical illness. Critical Care (London, England) 2006; 10:227.

99. Lewin B, ed. Genes V. New York: Oxford University Press and Cell Press; 1995.

100. Taudorf S, Krabbe KS, Berg RM, Moller K, Pedersen BK, Bruunsgaard H. Common studied polymorphisms do not affect plasma cytokine levels upon endotoxin exposure in humans. Clinical and Experimental Immunology 2008;152:147-52.

101. McCall CE, Yoza BK. Gene silencing in severe systemic inflammation. American Journal of Respiratory and Critical Care Medicine 2007;175:763-7.

102. Watanabe E, Hirasawa H, Oda S, Matsuda K, Hatano M, Tokuhisa T. Extremely high interleukin-6 blood levels and outcome in the critically ill are associated with tumor necrosis factor- and interleukin-1-related gene polymorphisms. Critical Care Medicine 2005;33:89-97; discussion 242-3.

103. Lewin B. Chapter 29. Building the transcription complex: promoters, factors, and RNA polymerases. In: Genes V. New York: Oxford University Press and Cell Press; 1995:847-77.

104. Ma P, Chen D, Pan J, Du B. Genomic polymorphism within interleukin-1 family cytokines influences the outcome of septic patients. Critical Care Medicine 2002;30:1046-50.

105. Jessen KM, Lindboe SB, Petersen AL, Eugen-Olsen J, Benfield T. Common TNF-alpha, IL-1 beta, PAI-1, uPA, CD14 and TLR4 polymorphisms are not associated with disease severity or outcome from gram negative sepsis. BMC Infectious Diseases 2007;7:108. 
106. Watanabe E, Hirasawa H, Oda S, et al. Cytokine-related genotypic differences in peak interleukin-6 blood levels of patients with SIRS and septic complications. The Journal of Trauma 2005;59:1181-9; discussion 9-90.

107. Wunderink RG, Waterer GW. Genetics of community-acquired pneumonia. Seminars in Respiratory and Critical Care Medicine 2005;26:553-62.

108. Sutherland AM, Walley KR, Manocha S, Russell JA. The association of interleukin 6 haplotype clades with mortality in critically ill adults. Archives of Internal Medicine 2005;165:75-82.

109. Barnes MR. Navigating the HapMap. Briefings in Bioinformatics 2006;7:211-24.

110. Tramont E. Host defense mechanisms: general or nonspecific host defense mechanisms. In: Mandell GL, Douglas RG, Jr., Bennett JE, eds. Principles and Practices of Infectious Diseases. New York, NY: Churchill Livingston Inc.; 1990:33-41.

111. Horan TC, Gaynes R. Surveillence of nosocomial infections. In: Mayhall CG, ed. Hospital epidemiology and infection control. Philadelphia, PA: Lippincott Williams \& Wilkins; 2004:1659-702.

112. Horan TC, Andrus M, Dudeck MA. CDC/NHSN surveillance definition of health care-associated infection and criteria for specific types of infections in the acute care setting. American Journal of Infection Control 2008;36:309-32.

113. Cannon JG, Nerad JL, Poutsiaka DD, Dinarello CA. Measuring circulating cytokines. Journal of Applied Physiology 1993;75:1897-902.

114. Fahey JL, Aziz N, Spritzler J, et al. Need for an external proficiency testing program for cytokines, chemokines, and plasma markers of immune activation. Clinical and Diagnostic Laboratory Immunology 2000;7:540-8.

115. Mire-Sluis AR, Das RG, Padilla A. WHO cytokine standardization: facilitating the development of cytokines in research, diagnosis and as therapeutic agents. Journal of Immunological Methods 1998;216:103-16.

116. Charlson ME, Pompei P, Ales KL, MacKenzie CR. A new method of classifying prognostic comorbidity in longitudinal studies: development and validation. Journal of Chronic Diseases 1987;40:373-83.

117. Aviles RJ, Martin DO, Apperson-Hansen C, et al. Inflammation as a risk factor for atrial fibrillation. Circulation 2003;108:3006-10. 
118. Boos CJ, Anderson RA, Lip GY. Is atrial fibrillation an inflammatory disorder? European Heart Journal 2006;27:136-49.

119. Schroder J, Kahlke V, Staubach KH, Zabel P, Stuber F. Gender differences in human sepsis. Archives of Surgery 1998;133:1200-5.

120. Mayr FB, Yende S, Linde-Zwirble WT, et al. Infection rate and acute organ dysfunction risk as explanations for racial differences in severe sepsis. Journal of the American Medical Association 2010;303:2495-503.

121. Klevay MJ, Ernst EJ, Hollanbaugh JL, Miller JG, Pfaller MA, Diekema DJ. Therapy and outcome of Candida glabrata versus Candida albicans bloodstream infection. Diagnostic Microbiology and Infectious Diseases 2008;60:273-7.

122. Mean M, Marchetti O, Calandra T. Bench-to-bedside review: Candida infections in the intensive care unit. Critical Care (London, England) 2008;12:204.

123. Lundstrom T, Sobel J. Nosocomial candiduria: a review. Clinical Infectious Diseases 2001;32:1602-7.

124. Cohen Y, Karoubi P, Adrie C, et al. Early prediction of Candida glabrata fungemia in nonneutropenic critically ill patients. Critical Care Medicine 2010;38:826-30.

125. Marik PE. Critical illness-related corticosteroid insufficiency. Chest 2009;135:181-93.

126. Meduri GU, Golden E, Freire AX, et al. Methylprednisolone infusion in early severe ARDS: results of a randomized controlled trial. Chest 2007;131:954-63.

127. Ramirez P, Ferrer M, Gimeno R, et al. Systemic inflammatory response and increased risk for ventilator-associated pneumonia: a preliminary study. Critical Care Medicine 2009;37:1691-5.

128. Baier RJ, Loggins J, Yanamandra K. IL-10, IL-6 and CD14 polymorphisms and sepsis outcome in ventilated very low birth weight infants. BMC Medicine 2006;4:10.

129. Zubert S, Funk DJ, Kumar A. Antibiotics in sepsis and septic shock: like everything else in life, timing is everything. Critical Care Medicine 2010;38:1211-2.

130. Kumar A. Optimizing antimicrobial therapy in sepsis and septic shock. Critical Care Nursing Clinics of North America 2011;23:79-97. 
131. Manginas A, Tsiavou A, Chaidaroglou A, et al. Inflammatory cytokine gene variants in coronary artery disease patients in Greece. Coronary Artery Disisease 2008;19:575-82. 


\section{APPENDIX A. VETERAN'S ADMINISTRATION MEDICAL CENTER INSTITUTIONAL REVIEW BOARD INITIAL APPROVAL}

mphis VA Medical Center (614)

1030 Jefferson Avenue • Memphis, TN $38104 \cdot 901-577-7267 \cdot$ Fax: 901-577-7273

IRB APPROVAL - Initial Review

Date: December 9, 2008

From: Barry M. Wall, M.D., Chairperson $\mathrm{ic}_{\mathrm{C}} \mathrm{f}$

Investigator: Reba A. Umberger, M.S., RN

Protocol: Inflammatory Proteins, Genetic variation, and Environmental Influences on Nosocomial Infection Development in Sepsis Patients

ID: 00617 Prom\#: N/A Protocol\#: N/A

The following items were reviewed and approved at the 11/26/2008 meeting:

- Consent Waiver - for screening only

- Abstract/PDS

- Advertisement - flyer

- Advertisement - information card

- Conflict of Interest

- Consent Form - HIPPA (11/17/2008)

- Training verification

- Request to Review Research Proposal/Project (11/17/2008)

- Key Personnel

- Protocol (11/17/2008)

- Human Studies Questionnaire

- Data Access Certifications \& Approvals (11/17/2008)

- Research Confidentiality \& Privacy Form (11/17/2008)

The following additional items were received to address stipulations and are now approved:

- Consent Form - Main (11/17/2008)

Approval is granted for a period of 12 months and will expire on 11/25/2009. Your Continuing Review is scheduled for $10 / 28 / 2009$.

The protocol was determined to have the following level of risk:

Minimal

The following other committee reviews are scheduled:

Research \& Development Committee [12/10/2008]

Approval by each of the following is required prior to study initiation:

Institutional Review Board

Research \& Development Committee

Page 1 of 2

The Memphis VAMC IRB is not connected with, has no authority over, and is not responsible for human research conducted at any other institution, except where a Memorandum of Understanding specifies otherwise. Separate consent forms, initial reviews, continuing reviews, amendments, and reporting of serious adverse events are required if the same study is conducted at multiple institutions. 
Approval for study initiation is contingent upon your compliance with the requirements of the Research Service for the conduct of studies involving human subjects.

Page 2 of 2

The Memphis VAMC IRB is not connected with, has no authority over, and is not responsible for human research conducted at

any other institution, except where a Memorandum of Understanding specifies otherwise. Separate consent forms, initial reviews,

continuing reviews, amendments, and reporting of serious adverse events are required if the same study is conducted at multiple

institutions. 


\title{
APPENDIX B. VETERAN'S ADMINISTRATION RESEARCH AND DEVELOPMENT COMMITTEE ANNUAL REVIEW
}

\author{
Research \& Development Committee \\ Memphis VA Medical Center (614) \\ 1030 Jefferson Avenue • Memphis, TN $38104 \cdot 901-577-7267 \cdot$ Fax: 901-577-7273
}

\section{APPROVAL - Annual Review}

Date: February 12, 2010
From: James M. Fleckenstein, M.D., Chairperson f $f$

Investigator: Reba A. Umberger, M.S., RN

Protocol: Inflammatory Proteins, Genetic variation, and Environmental Influences on Nosocomial Infection Development in Sepsis Patients

ID: 00617 Prom\#: N/A Protocol\#: N/A

The following items were reviewed and approved at the 02/10/2010 meeting:

- Continuing Review Form - Annual R\&D review (01/26/2010) 


\section{APPENDIX C. THE UNIVERSITY OF TENNESSEE HEALTH SCIENCE CENTER INSTITUTIONAL REVIEW BOARD INITIAL APPROVAL}

\begin{tabular}{lr}
\hline THE UNIVERSITY OF TENNESSEE \\
Health Science Center \\
\hline \\
Institutional Review Board \\
910 Madison Avenue, Suite 600 \\
Memphis, TN 38163 \\
Tel: (901) 448-4824
\end{tabular}

January 2, 2009

Reba Umberger

College of Nursing

Department of Nursing - Academic Programs

Coleman Building

Suite H314

CAMPUS

Re: IRB\# 08-00197-XP: Study Title: Inflammatory proteins, genetic variation, and environmental influences on nosocomial infection devel opment in sepsis patients

Dear Ms. Umberger,

The IRB has received your written acceptance of and/or response dated December 13, 2008 to the provisos outlingd in my letter of December 10,2008 concerring the above referenced project.

The IRB determined your application to be consistent with the guidelines for expectited review under categories (2), (3) and (5). The IRB has reviewed these materials and determined that they do comply with proper consideration for the rights and welf are of human subjects and the regulatory requirements for the protection of human subjects. Therefore, this letter constitutes full approval by the IRB of your application as submitted including flyer, data collection form; study recruitment card [page 1/page2]; main consent form and surrogate consent form (stamped IRB approved on January 2, 2009). This study was approved for 12 months with an expiration date of December 10, 2009 .

The IRB has determined that the informed consent form, incorporating the authorization of subjects to use their protected health inf ormation in research, complies with the federal privacy regulations as specified in 45 CFR 160 and 45 CFR 164.

In the event that subjects are to be recruited using solicitation materials, such as brochures, posters, webbased advertisements, etc., these materials must receive prior approval of the IRB. Any revisions in the approved application must also be submitted to and approved by the IRB prior to implementation. In addition you are responsible for reporting any unanticipated serious adverse events or other problems involving risks to subjects or others in the manner required by the local IRB policy.

Finally, re-approval of your project is required by the IRB in accord with the conditions specified above. You may not continue the research study beyond the time or other limits specified unless you obtain prior written approval of the IRB.

Sincerely,

Signature applied by Clair E Cox on 01/02/2009 10:36:52 AM CS T

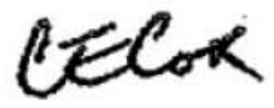

Signature applied by Donna L Stallings on 01/02/2009 10:38:23 AM CS T 


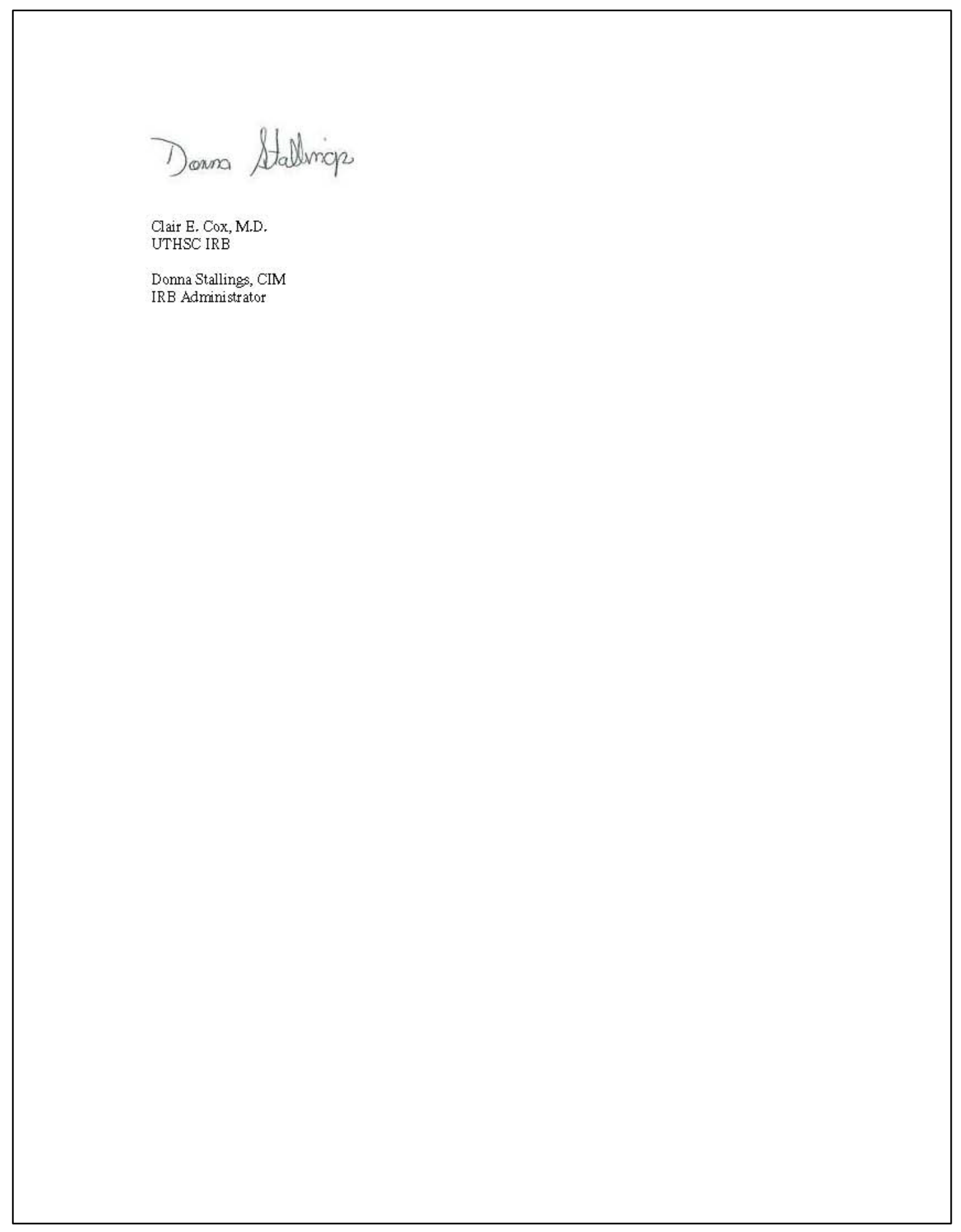


Title of Study: Inflammatory Proteins, Genetic Variation, and Environmental Influences on Nosocomial Infection Development in Sepsis Patients

Principal Investigator: Reba Umberger, MS, RN

VAMC: Memphis TN

Subject Name:

Date:

\section{Research Statement}

You are being given the opportunity to participate in a research study which includes genetic analysis. Research studies include only people who choose to take part. Please read this consent form carefully and take your time making your decision. As your study investigator discusses this consent form with you, please ask her to explain any words or information that you do not clearly understand. We encourage you to talk with your family and friends before you decide to take part in this research study. The nature of the study, risks, inconveniences, discomforts, and other important information about the study are listed below.

The purpose of this study is to gain a better understanding of the causes of infection that occur in the intensive care unit. You are being asked to participate because you already have an infection or suspected infection of some kind and we would like to learn about risk factors for developing additional infections while you are in the intensive care unit. This involves examining protein levels in your blood and examining genes involved in the way your body responds to infection. It also involves collecting information about your progress and health while you are in the intensive care unit.

Genes are like blueprints in each of our cells that determine traits that we inherit, like eye color and hair color. Genes may also influence what diseases we get and how we respond to treatment. DNA is the substance that makes up our genes.

Approximately 78 subjects will be participating in this study locally. The study will take place at the Veteran's Affairs Medical Center, Memphis and The University of Tennessee Health Science Center.

Your participation in this study will last until you have been discharged from the intensive care unit for at least 2 days (or as long as 28 days if you are in the intensive care for a longer period).

\section{Procedures to be followed}

If you choose to participate in this research study, approximately 1 teaspoon of blood will be drawn from your arm and a cotton swab will be used to gently rub the inside of your cheek. This blood sample will be used to measure protein levels of your immune response. The cheek swab will be used to extraction your DNA to examine genes involved in your immune system. Data about your clinical care including your medical history, reason for your current admission will be collected and all laboratory tests, vital signs, special procedures (such as x-rays), medications, physical assessments, and other information pertaining to your hospital stay will be collected daily until you complete the study.

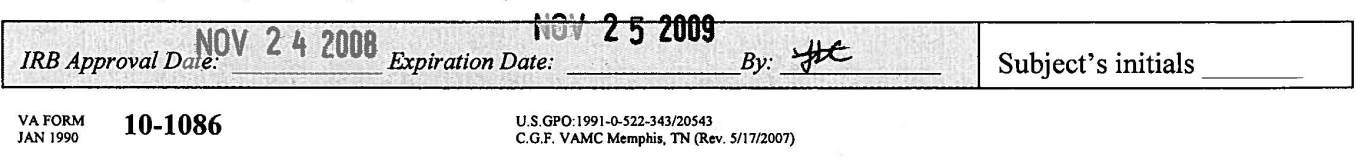


Title of Study: Inflammatory Proteins, Genetic Variation, and Environmental Influences on Nosocomial Infection Development in Sepsis Patients

Your blood sample, DNA specimen, and associated data will become the property of the principle investigator. Your specimens will be retained until the study is completed. At the completion of the research, your blood sample and DNA specimen will be destroyed. Your specimens will not be used in any profit making activity. Due to the experimental nature of any research that is conducted with your blood and cheek samples, you will not be informed of any individual results.

After you complete the study, you will not be re-contacted regarding your health status.

\section{Reasonably Foreseeable Risks or Discomforts}

The potential risks associated with drawing blood from your arm include minimal discomfort and/or bruising. Infection, excess bleeding/bruising, clotting, lightheadedness and/or fainting are also possible, although unlikely.

There is a potential risk of unintended disclosure of confidential information to parties outside of the research study that might affect your ability to get insurance or a job. However, these risks are quite remote since appropriate confidentiality measures will be taken to protect any information about your health that is revealed by your DNA sample.

\section{Reasonably Expected Benefits to Subjects or Others}

There are no direct benefits for participating in this research study. Your participation in this research study may provide additional information regarding the possible causes of infections occurring in the intensive care unit which may be of help to future patients.

\section{Appropriate Alternatives}

You have the option of not participating in this research study. You will receive the same medical care whether or not you participate in this research study.

\section{Confidentiality and Authorization for Release of Protected Health Information for Research Purposes}

All your paper research records will be stored in locked file cabinets and will be accessible only to research personnel. All your electronic research records will be computer password protected and accessible only to research personnel.

Your blood and cheek swab sample will be de-identified to protect your identity prior to being sent for analysis in a laboratory on the UTHSC campus. The data collected at this institution will also be labeled with a code. The master key that links your name with the code on the research record/ specimens will be maintained in a locked cabinet and in a secure folder on the server at the VAMC, Memphis.

Under federal privacy regulations, you have the right to determine who has access to your personal health information (called "protected health information" or PHI). By signing this document, you will authorize the Veterans Health Administration (VHA) to provide Reba

\begin{tabular}{|l|l|l|}
\hline IRB A proval Date. & NOV $24200 Q_{\text {ExpirationiDateNOV } 252009 \text { By: the }}$ Subject's initials \\
\hline
\end{tabular}

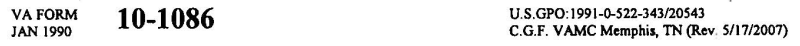


Title of Study: Inflammatory Proteins, Genetic Variation, and Environmental Influences on Nosocomial Infection Development in Sepsis Patients

Umberger and her research team to use and disclosure the following information. PHI collected in this study may include your medical history, the results of physical exams, lab tests, $\mathrm{x}$-ray exams, and other diagnostic and treatment procedures, as well as basic demographic information. By signing this consent form, you are authorizing the researchers at the VAMC, Memphis to have access to your PHI and to receive PHI from your physician and facilities where you have received health care. The information that will be released includes all information on your chart and may include information regarding Drug Abuse, Alcoholism, Results of testing for Infection with Human Immunodeficiency Virus (HIV), and Sickle cell anemia if it is part of your health history.

A copy of this consent form will be placed in your medical record. The results of this study may be published, but your records will not be revealed unless required by law. The results of genetic testing and blood test preformed for the study will not appear in your medical record. You will not be identified in any presentations or publications based on the results of the research study.

The research team may also need to disclose the information to others as part of the study process. The others may include the Institutional Review Board (IRB) that will monitor this study.

If you do not agree with this authorization, you will not participate in the study and should not sign this form. You will be given a signed copy of this form for your records. You authorize the use of your identifiable information as described in this form. This authorization to use your information will expire at the end of the research study.

You can revoke this authorization, in writing, at any time. To revoke your authorization, you must write to the Release of Information Office at this facility or you can ask a member of the research team to give you a form to revoke the authorization. Your request will be valid when the Release of Information Office receives it. If you revoke this authorization, you will not be able to continue to participate in the study. This will not affect your right as a VHA patient to treatment or benefits outside the study.

If you revoke this authorization, Reba Umberger and her research team can continue to use information about you that was collected before receipt of the revocation. The research team will not collect information about you after you revoke the authorization.

The VHA complies with the requirements of the Health Insurance Portability and Accountability Act of 1996 and its privacy regulations and all other applicable laws that protect your privacy. We will protect your information according to these laws. Despite these protections, there is a possibility that your information could be used or disclosed in a way that it will no longer be protected. Our Notice of Privacy Practices (a separate document) provides more information on

IRB Approval Date: NOV 242002 piration Date:NOV 252009 By: ft

Subject's initials 
Title of Study: Inflammatory Proteins, Genetic Variation, and Environmental Influences on Nosocomial Infection Development in Sepsis Patients

how we protect your information. If you do not have a copy of the Notice, the research team will provide one to you.

\section{Compensation or Treatment for Injury}

If any medical problems occur in connection with this study the VA medical facility shall provide necessary medical treatment to you as a research subject injured as a result of participation in a research project approved by the VA R\&D Committee and conducted under the supervision of one or more VA employees in accordance with Federal regulations.

In case there are medical problems or questions, you can call: Reba Umberger at

This is a cell number which can be reached 24-hours/7-days per week. Cell phone service may be blocked in certain area of the hospital; therefore, please leave a message if there is no answer and your call will be returned as promptly as possible.

If you have any further questions about your rights as a research subject, you may call the Memphis Research Office at (901) 577-7267.

\section{Additional Costs}

There is no cost to you for participating in this research study and no payment for participation. This research study involves only observation of your care and no treatment is involved. Veterans in some categories may be subject to co-payments, if indicated by a means test, in accordance with Federal regulations. This research study does not affect any cost that would be incurred if you had not participated in this study.

\section{Early Withdrawal/Procedures for Termination}

Your participation in this research study is completely voluntary. You may choose not to participate without any loss of benefits to which you are otherwise entitled.

If you give your permission to use your DNA sample for the research specified above, and then you decide later, that you do not want the specimen to be used, you may contact the investigator and indicate your wishes in writing. If you withdraw from this research study, your specimen and related data will be destroyed; however, any analysis that was completed before you chose to withdraw from the research study will be retained if it is necessary for completion of the research. 
Department of Veterans Affairs

VA RESEARCH CONSENT FORM

Page 5 of 5

Title of Study: Inflammatory Proteins, Genetic Variation, and Environmental Influences on Nosocomial Infection Development in Sepsis Patients

\section{RESEARCH SUBJECT'S RIGHTS:}

I have read or have had read to me all of the above. has

explained the study to me and answered all of my questions. I have been told of the risks or discomforts and possible benefits of the study. I have been told of other choices of treatment available to me.

I understand that I do not have to take part in this study, and my refusal to participate will involve no penalty or loss of rights to which I am entitled. I may withdraw from this study at any time without penalty or loss of VA or other benefits to which I am entitled. I

voluntarily consent to participate in this study. I understand what the study is about and how and why it is being done.

Subject's Signature

Signature of Witness (not associated with study)

Signature of Person Obtaining Consent

\section{Date}

Witness (Print Name \& Date)

Date

Print Name of Person Obtaining Consent

\begin{tabular}{|l|l|l|}
\hline IRB Approval Date & NOV $242008_{\text {Expiration Date: }}$ NOV 252009 By: ffe & Subject's initials \\
\hline
\end{tabular}




\section{Department of Veterans Affairs}

VA RESEARCH CONSENT FORM

Page 1 of 1

Title of Study: Title of Study: Inflammatory Proteins, Genetic Variation, and Environmental Influences on Nosocomial Infection Development in Sepsis Patients

Principal Investigator: Reba Umberger, MS, RN

VAMC: Memphis TN

Subject Name:

Date:

\section{Surrogate Decision Maker Consent}

In the event the patient is unable to consent for himself/herself, and the Investigational treatment as outlined in the attached consent form is an available treatment option, consent may be obtained from a surrogate decision maker. A surrogate is an individual who is (1). Appointed under the Durable Power of Attorney for Health Care, (2). A court-appointed guardian, or (3). A relative actively involved in the life of the patient and who appears to be acting in the best interests of the patient, so that the surrogate's decision would reasonably be considered what the patient would have chosen to do. A surrogate may be a relative in the following order of priority: spouse, adult son or daughter, parent, or adult brother or sister. Other relatives are not eligible to serve as surrogate decision makers.

STATEMENT OF SURROGATE DECISION MAKER:

I,

am the

(Relationship to patient) of (patient name). I have read and understand this consent form. I have had the opportunity to ask questions, and all of my questions have been answered. I understand that this study does not involve an investigational treatment. I believe this study is in his/her best interests and is consistent with what she/he would have decided had she/he been able to do so.

Signature of Surrogate Decision Maker

Signature of Witness (not associated with study)

Signature of Person Obtaining Consent

\section{Date}

Print Name of Person Obtaining Consent

Subject's initials 


\title{
APPENDIX E. DATA COLLECTION FORMS
}

\author{
Inflammatory Proteins, Genetic Variation, and Environmental Influences on Nosocomial \\ Infection Development in Sepsis Patients
}

VAMC, IRB \#, PI: Umberger

\section{LIST OF STUDY FoRMS}

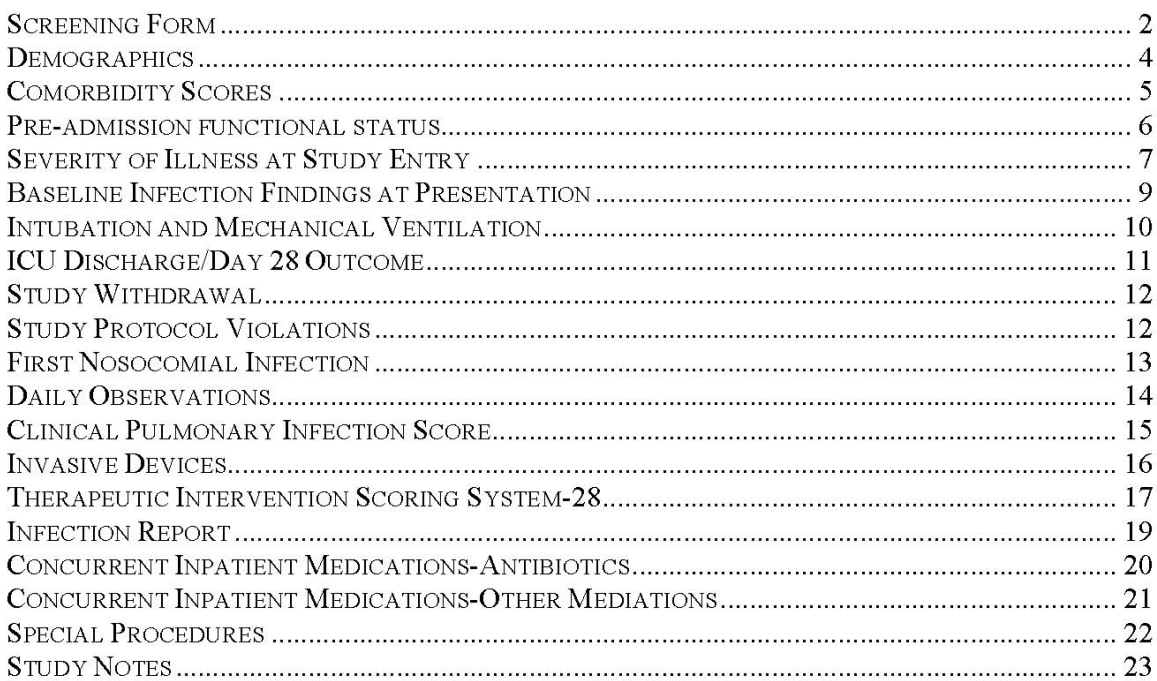


Inflammatory Proteins, Genetic Variation, and Environmental Influences on Nosocomial Infection Development in Sepsis Patients

VAMC, IRB \#, PI: Umberger

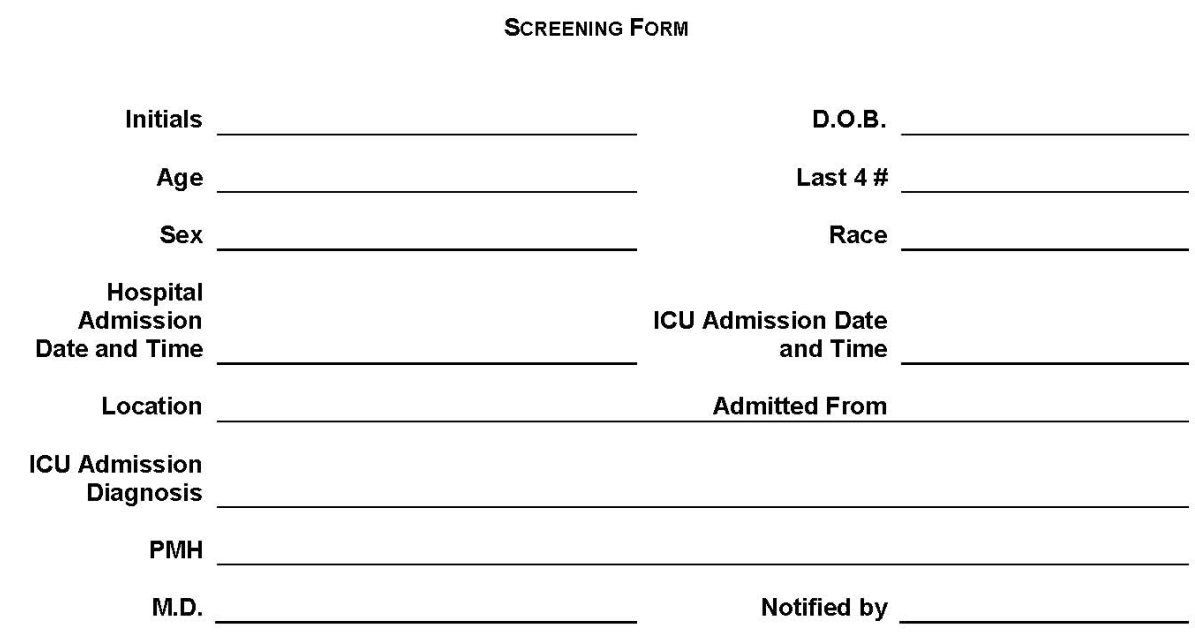

Inclusion Criteria

Presence of Sepsis within 72 hours of admission to the ICU: Sepsis is defined as Systemic inflammatory response syndrome (SIRS) plus an identified or suspected infection .

Infection: definitive or suspected

Describe

SIRS Score of at least 2 :

elevated temperature $\left(>38^{\circ} \mathrm{C}\right.$ or $\left.<36^{\circ} \mathrm{C}\right)$,

minimum heart rate $(>90$ per minute)

elevated respiratory rate $\left(>20\right.$ per minute) or a $\mathrm{PaCO}_{2}$ of $<32 \mathrm{mmHg}$, white blood cell count $\left(>12,000\right.$ or $<4,000$ cells $\mu \mathrm{L}^{-1}$ ).

1. Date/time when sepsis developed:

2. Age 18 or older.

\section{Exclusion Criteria:}

Receiving immunosuppressive agents (chemotherapy or $>1 \mathrm{mg} / \mathrm{kg}$ of prednisone/

1. equivalent dose within the past 3 months)

Other Immunosuppression: ADS

Liver failure (Child-Pugh $\geq$ category C )

Lack of informed consent 
Inflammatory Proteins, Genetic Variation, and Environmental Influences on Nosocomial Infection Development in Sepsis Patients

VAMC, IRB \#, PI: Umberger

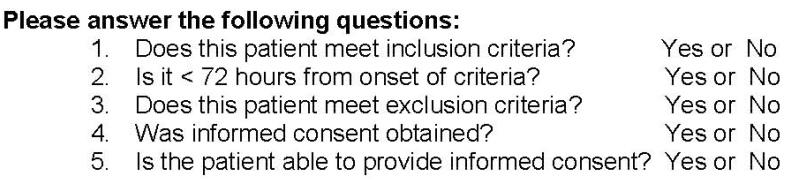

1. Does this patient meet inclusion criteria? Yes or No

2. Is it $<72$ hours from onset of criteria? Yes or No

3. Does this patient meet exclusion criteria? Yes or No

4. Was informed consent obtained? Yes or No

5. Is the patient able to provide informed consent? Yes or No

If the patient meets inclusion criteria, has no exclusion, and is within the enrollment window, obtain informed consent by patient or legally authorized representative.

Informed consent: $\quad \square \quad$ original retained with research records

$\square \quad$ copy given to patient or LAR, and

$\square \quad$ copy placed on chart

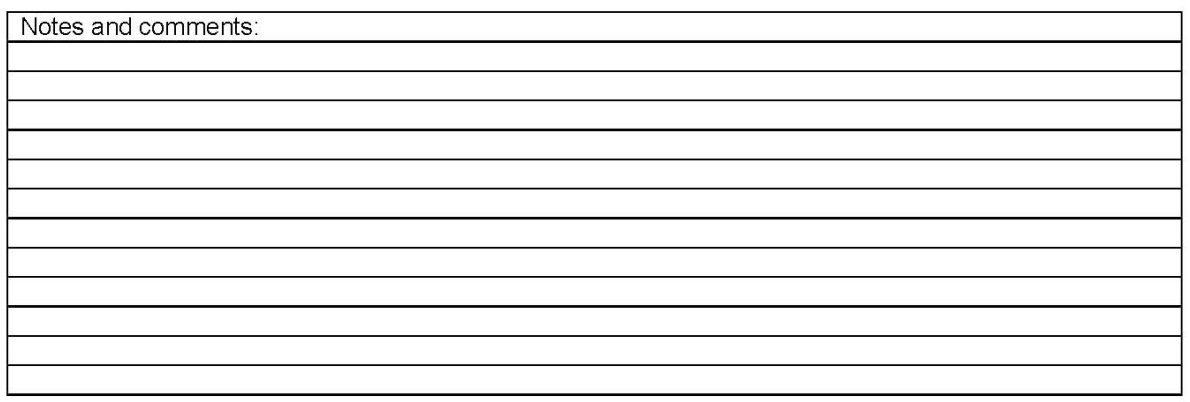

Name of screener

Date

Child-Pugh Classification of Chronic Liver Disease

\begin{tabular}{|c|c|c|c|}
\hline Variable & 1 Point & 2 Points & 3 Points \\
\hline Ascites & Absent & Present & Tense \\
\hline Encephalopathy & None & Grade I or II & Grade III or IV \\
\hline Serum albumin, g/dl & $>3.5$ & 2.8 to 3.5 & $<2.8$ \\
\hline Serum bilirubin, $\mu \mathrm{mol} / \mathrm{dl}$ & $<3.4$ & 3.4 to 5.1 & $>5.1$ \\
\hline $\begin{array}{l}\text { Prothrombin time, s above } \\
\text { control }\end{array}$ & $<4.0$ & 4.0 to 6.0 & $>6.0$ \\
\hline $\begin{array}{ll}5-6= & m \\
7-9= & m \\
10-15= & \mathrm{r}\end{array}$ & $\begin{array}{l}\text { (Child } \\
\text { lisease (C } \\
\text { ease (Chi }\end{array}$ & $\begin{array}{l}\text { Iss B) } \\
\text { C) }\end{array}$ & \\
\hline
\end{tabular}

Encephalopathy:

Grade I-Detectable but slight euphoria, anxiety, or lack of awareness, shortened attention span, difficulty with arithmetic;

Grade II-Lethargy, disorientation for time, change of personality, uncharacteristic or inappropriate behavior;

GradeIII-Somnolence to semi-stupor but response to stimuli, confusion, gross disorientation;

Grade IV-Coma 
Inflammatory Proteins, Genetic Variation, and Environmental Influences on Nosocomial Infection Development in Sepsis Patients

VAMC, IRB \#, PI: Umberger

DEMOGRAPHICS

1. Study Number

2. Study Entry Date

3. Name (Initials)

6. Hospital Admission Date

7. ICU Admission Date

8. Age

9. Sex

10. Race (Hispanic or Latino) Yes or No or Unknown

Race (1=American Indian/Alaska Native, 2=Asian, 3=Native

Hawaiian or Other Pacific Islander, 4=Black or African American,

11. $5=$ White, $6=$ More Than One Race, $7=$ Unknown or Not reported)

12. Weight $(\mathrm{kg})$

13. Height (cm)

14. Body mass index

15. Primary reason for ICU admission (list)

16. ICU admission diagnosis

17. Past Medical History

18. Prior admissions to the ICU for an infection/sepsis?

19. Prior admissions with septic shock/use of vasopressors?

20. Date sepsis criteria med (see screener form)
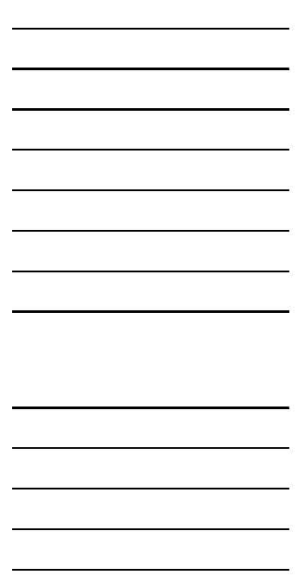

Prior admissions to the ICU for an infection/sepsis?


Inflammatory Proteins, Genetic Variation, and Environmental Influences on Nosocomial Infection Development in Sepsis Patients

VAMC, IRB \#, PI: Umberger

\begin{tabular}{|c|c|c|c|}
\hline \multicolumn{4}{|c|}{ COMORBIDITY SCORES } \\
\hline 1. & Charlson Weighted Index of Comorbidity & & $\begin{array}{l}\text { Sum of } \\
\text { scores } \\
\text { below }\end{array}$ \\
\hline a) & Myocardial infarction & Yes _ No & 1 \\
\hline b) & Congestive Heart Failure & Yes __ No & 1 \\
\hline c) & Peripheral vascular disease & Yes _ No & 1 \\
\hline d) & Cerebrovascular disease & Yes _ No_ & 1 \\
\hline e) & Dementia & Yes _ No & 1 \\
\hline f) & Chronic Pulmonary Disease & Yes _ No & 1 \\
\hline g) & Connective Tissue disease & Yes _ No_ & 1 \\
\hline h) & Ulcer disease & Yes _ No_ & 1 \\
\hline i) & Mild Liver disease & Yes _ No_ & 1 \\
\hline j) & Diabetes & Yes _ No & 1 \\
\hline k) & Hemiplegia & Yes _ No & 2 \\
\hline l) & Moderate or severe renal disease & Yes _ N No_ & 2 \\
\hline m) & Diabetes with end organ damage & Yes __ No_ & 2 \\
\hline n) & Any tumor & Yes ___ No & 2 \\
\hline 0) & Leukemia & Yes _ No & 2 \\
\hline p) & Lymphoma & Yes __ No_ & 2 \\
\hline q) & Moderate or severe liver disease & Yes _ N No_ & 3 \\
\hline г) & Metastatic solid tumor & Yes __ No & 6 \\
\hline \multirow[t]{2}{*}{ s) } & AIDS & Yes__ No___ & 6 \\
\hline & & SUM & \\
\hline $1 b$. & Charlson comorbidity index $>0$ & Number & $(\%)$ \\
\hline
\end{tabular}

2. Social History

a) Drug abuse

b) Current smoker

c) Lifetime non-smoker

d) Smoke free for last 5 years

e) Pack years history

f) Alcohol abuse

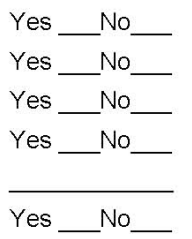

3. Atherosclerotic CV disease risk factors score

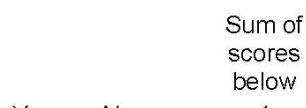

a) - Smoker

Yes _No below 
Inflammatory Proteins, Genetic Variation, and Environmental Influences on Nosocomial Infection Development in Sepsis Patients

VAMC, IRB \#, PI: Umberger

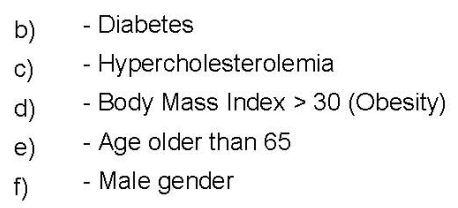

\begin{tabular}{ll} 
Yes_No___ & 1 \\
Yes_No___ & 1 \\
Yes_No__ & 1 \\
Yes_No__ & 1 \\
Yes_No_ & 1 \\
\hline SUM
\end{tabular}

Pre-ADMISSION FUnCTIONAL STATUS

\begin{tabular}{llc}
\hline 1. & Karnofsky performance score & $\begin{array}{c}\text { Select } \\
\text { One }\end{array}$ \\
\cline { 2 - 2 } a) & Normal, no complaints, no signs of disease & $100 \%$ \\
b) & Capable of normal activity, few symptoms or signs of disease & $90 \%$ \\
c) & Normal activity with some difficulty, some symptoms or signs & $80 \%$ \\
d) & Caring for self, not capable of normal activity or work & $70 \%$ \\
e) & Requiring some help, can take care of most personal requirements & $60 \%$ \\
f) & Requires help often, requires frequent medical care & $50 \%$ \\
g) & Disabled, requires special care and help & $40 \%$ \\
h) & Severely disabled, hospital admission indicated but no risk of death & $30 \%$ \\
i) & Very ill, urgently requiring admission, requires supportive measures or & $20 \%$ \\
j) & Moribund, rapidly progressive fatal disease processes & $10 \%$ \\
k) & Karnofsky Score &
\end{tabular}


Inflammatory Proteins, Genetic Variation, and Environmental Influences on Nosocomial Infection Development in Sepsis Patients

VAMC, IRB \#, PI: Umberger

ID\# Initials

\section{Medications}

\begin{tabular}{|c|c|c|}
\hline a) & Antibiotics use prior to ICU admission (list on medication form) & Yes _. No \\
\hline b) & Any steroids use prior to entry $(<1 \mathrm{mg} / \mathrm{kr})$ & Yes No No \\
\hline c) & Any steroids use after study entry (list on medication form with reason) & Yes _. No \\
\hline d) & Diabetic requiring Insulin prior to entry? AdmHgBA1C if done & Yes \\
\hline e) & Required insulin during ICU stay? & Yes _ No \\
\hline
\end{tabular}

SEVERITY OF ILLNESS AT STUdY ENTRY

\begin{tabular}{|c|c|c|}
\hline \multirow[t]{2}{*}{1} & APACHE scores at ICU entry & \\
\hline & Insert the worst values within 24 hours of ICU admission: & lowest and highest \\
\hline a. & Temperature & \\
\hline b. & Systolic BP & \\
\hline c. & Diastolic BP & \\
\hline d. & Heart Rate & \\
\hline e. & Respiratory Rate & \\
\hline f. & Sodium & \\
\hline g. & Potassium & \\
\hline h. & Glucose & \\
\hline i. & Creatinine & \\
\hline j. & BUN & \\
\hline k. & Urine output over 24 hours & \\
\hline I. & Albumin & \\
\hline $\mathrm{m}$. & Bilirubin & \\
\hline n. & Hematocrit $(\%)$ & \\
\hline 0. & White blood cell count & \\
\hline $\mathrm{p}$. & $\mathrm{FiO} 2(\%)$ & \\
\hline q. & $\mathrm{pH}$ & \\
\hline r. & pO2 & \\
\hline s. & $\mathrm{pCO} 2$ & \\
\hline $\mathrm{t}$ & $\mathrm{HCO} 3$ & \\
\hline u. & GCS & \\
\hline v. & Age score & \\
\hline w. & Presence of Acute Renal Failure & \\
\hline$x$ & Chronic Health Score & \\
\hline$y$. & ICU admission score: & \\
\hline A. & APACHEIV Score http://www.icumedicus.com/icu_scores/apachelV.php & \\
\hline & APACHE IV APS Score & \\
\hline & APACHE IV Predicted Mortality Rate & \\
\hline & APACHE IV Predicted ICU Length of Stay & \\
\hline
\end{tabular}


Inflammatory Proteins, Genetic Variation, and Environmental Influences on Nosocomial Infection Development in Sepsis Patients

VAMC, IRB \#, PI: Umberger

ID\# Initials

Print and retain these calculations

B. APACHE II score http://www.icumedicus.com/icu_scores/apache.php APACHE II APS Score

APACHE II Predicted Mortality Rate

Print and retain these calculations

2 Organ Failure scores

a. SOFA score http://www. icumedicus.com/icu_scores/sofa.php

b. MODS score http://www icumedicus.com/icu_scores/mods.php

Print and retain these calculations at baseline

3 Other Mortality Prediction Model

a. MPMO score http://www.icumedicus.com/icu_scores/mpm0.php

b. MPM24 scorehttp://www icumedicus.com/icu_scores/mpm24 php

Print and retain these calculations 
Inflammatory Proteins, Genetic Variation, and Environmental Influences on Nosocomial Infection Development in Sepsis Patients

VAMC, IRB \#, PI: Umberger

ID\#

Initials

Baseline Infection Findings at Presentation

\begin{tabular}{|c|c|c|}
\hline 1 & Infection suspected or definitive? & \\
\hline 2 & Site of infection & \\
\hline 3 & Type of infection & \\
\hline 4 & SIRS Score at baseline & \\
\hline 5 & Temperature, ${ }^{\circ} \mathrm{F}$ & \\
\hline 6 & WBC count, $\times 10^{9} / \mathrm{L}$ & \\
\hline 7 & Respiratory Rate/Minute ventilation & \\
\hline 8 & Heart rate minimum & \\
\hline 9 & Minimum SBP & \\
\hline 10 & Number of fluid boluses (if any) required at admission & \\
\hline 11 & Use of vasopressors required? & Yes_No_ \\
\hline 12 & Max dose of Levophed first 24 hours (if required) & \\
\hline 13 & Chest radiograph score $(1-4)$ & \\
\hline 14 & Chest radiograph with bilateral involvement & \\
\hline 15 & $\mathrm{PaO}_{2}: \mathrm{FiO}_{2}$ & \\
\hline 16 & Blood cultures obtained & Yes_No_ No \\
\hline 17 & Were blood cultures obtained prior to antibiotics? & Yes_No_ \\
\hline 18 & UA and Urine Cultures obtained? & Yes_No_ No \\
\hline 19 & Sputum culture obtained & Yes_No_ \\
\hline 20 & Endotracheal aspirate obtained (ventilated pts) & Yes_No_ No \\
\hline 21 & BAL sample obtained & Yes \\
\hline 22 & C-reactive protein level & \\
\hline 23 & Procalcitonin level (not clinically available at this time) & \\
\hline 24 & Timing from meeting criteria until ICU admission? & \\
\hline 25 & Timing from meeting criteria until initiation of antibiotic treatment (hrs) & \\
\hline 26 & Date and Time first antibiotic was received & \\
\hline 27 & Initial appropriate antibiotic treatment & \\
\hline 28 & Surgical treatment required? & \\
\hline 29 & Other treatment of infection? & \\
\hline 30 & Conventional mechanical ventilation at study entry? & Yes__ No ___ \\
\hline 31 & Noninvasive positive pressure ventilation at study entry? & Yes__ No _ _ \\
\hline
\end{tabular}


Inflammatory Proteins, Genetic Variation, and Environmental Influences on Nosocomial Infection Development in Sepsis Patients

Intubation and Mechanical Ventilation

Was the patient intubated at any time during ICU stay (up to

1. 28 days)?

2. Date of Intubation

3. Date of Extubation

4. Was extubation successful (lasting at least 48 hours?)

5. Duration of Mechanical Ventilation

Yes or No

$\mathrm{mm} / \mathrm{dd} / \mathrm{yyyy}$

$\mathrm{mm} / \mathrm{dd} / \mathrm{yyyy}$

Did the patient receive non-invasive face mask ventilation?

FMV Beginning Date

FMV Ending Date

Yes or No

Duration of FM Ventilation

Days

Reintubated for any reason after extubation?

11. Date of Reintubation

Yes or No

$\mathrm{mm} / \mathrm{dd} /$ /yyy

$\mathrm{mm} / \mathrm{dd} / \mathrm{yyyy}$

Days

Yes or No

$\mathrm{mm} / \mathrm{dd} / \mathrm{yy} y \mathrm{y}$

12. Date of Subsequent Extubation $\mathrm{mm} / \mathrm{dd} / \mathrm{yyyy}$

Duration of any subsequent mechanical ventilation requiring

13. intubation (if at least 48 hours between)

Did patient have ARDS/ALI at any time during ICU stay (up

14. to 28 days)?

15. Mechanical Ventilation Free Days to Day 28

Note if patients are discharged prior to day 28 , much check CPRS to assure that no admissions occur in the subsequent period)

Days

Yes or No

Days 
Inflammatory Proteins, Genetic Variation, and Environmental Influences on Nosocomial Infection Development in Sepsis Patients

VAMC, IRB \#, PI: Umberger

ID\#

Initials

ICU DISCHARGE/DAY 28 OUTCOME

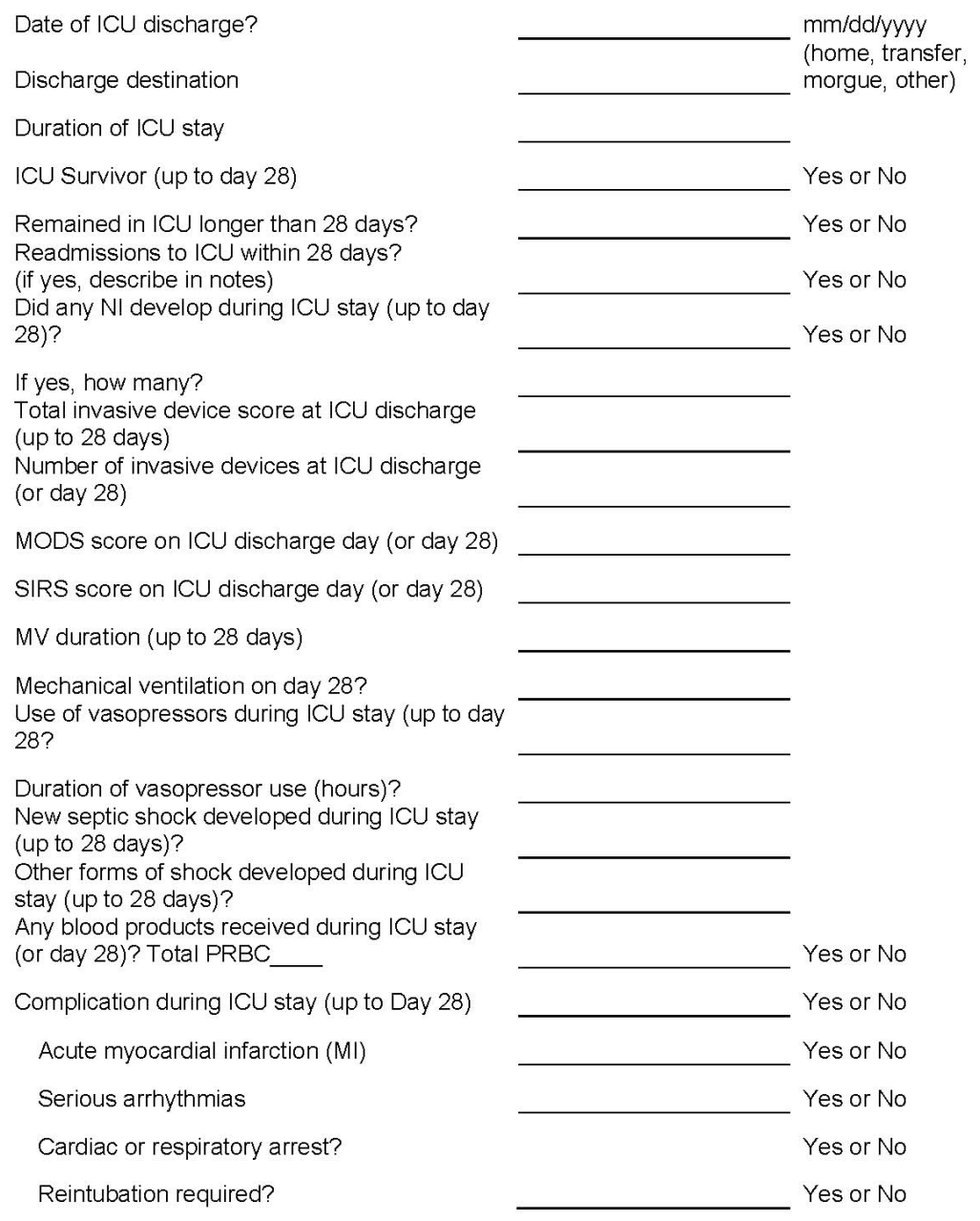

- Data collection is limited to 28 days, collect value up to 28 days for longer admissions.

- Consider first ICU admission for those readmitted but continue to make relevant notes up to 28 days. 
Inflammatory Proteins, Genetic Variation, and Environmental Influences on Nosocomial Infection Development in Sepsis Patients

VAMC, IRB \#, PI: Umberger

ID\# Initials

\section{STUDY WITHDRAWAL}

Did the patient withdraw from the study?

Yes/No

Withdrawal date:

Reason for withdrawal:

STUdY PROTOCOL VIOLATIONS

Have there been any protocol violation or approved exceptions during this study? Yes/No

If yes:

Describe violation

Persons notified

- IRB Report filed 
Inflammatory Proteins, Genetic Variation, and Environmental Influences on Nosocomial Infection Development in Sepsis Patients

VAMC, IRB \#, PI: Umberger

ID\#

Initials

FIRST NOSOCOMIAL INFECTION

\begin{tabular}{|c|c|c|}
\hline 1 & $\mathrm{NI}$ developed during first 28 days of ICU stay? & Yes__No___ \\
\hline & IF NO NI DEVELOPED SKIP TO ICU DISCHARGEIDAY 28 OUTCOME & \\
\hline 2 & Day of first NI & \\
\hline 3 & Site of infection & \\
\hline 4 & Type of infection & \\
\hline 5 & Mixed (polymicrobial infection) & Yes__No___ \\
\hline 6 & Multiple site infection & Yes__ No__ \\
\hline 7 & Antibiotic resistant organism? & Yes _ \\
\hline 8 & Infection confirmed with consultant (not colonization) & Yes_No_ No \\
\hline 9 & Invasive device score at time of first NI (see list) & \\
\hline 10 & MV duration at time of first $\mathrm{N}$ & \\
\hline 11 & Length of ICU stay at time of first $\mathrm{NI}$ & \\
\hline 12 & MODS score at time of first $\mathrm{NI}$ & \\
\hline 13 & SIRS Score on day of first $\mathrm{NI}$ & \\
\hline 14 & -Temperature max on day of first $\mathrm{NI},{ }^{\circ} \mathrm{F}$ & \\
\hline 15 & - Max WBC count on day of first $\mathrm{NI}, \times 10^{9} / \mathrm{L}$ & \\
\hline 16 & -Respiratory Rate/Minute ventilation & \\
\hline 17 & -Heart rate minimum & \\
\hline 18 & Minimum SBP on day of first $\mathrm{NI}$ & \\
\hline 19 & Any fluid boluses required on day of first NI? & \\
\hline 20 & Use of vasopressors required on day of first NI? & Yes_No_ No \\
\hline 21 & Max dose of levophed first on day if first NI (if required) & \\
\hline 22 & Chest radiograph score (1-4) & \\
\hline 23 & Chest radiograph with bilateral involvement & Yes_No_NA_ \\
\hline 24 & $\mathrm{PaO}_{2}: \mathrm{FiO}_{2}$ & \\
\hline 25 & CPIS Score at time of first $\mathrm{NI}$ & \\
\hline 26 & Change in antibiotics & Yes \\
\hline 28 & Surgical treatment required? (see special procedures) & Yes__ No ___ \\
\hline 29 & Other treatment of infection? (see special procedures) & Yes__No_ No \\
\hline 30 & On $\mathrm{MV}$ at time of first NI? & Yes__ No _ \\
\hline 31 & Central line present at time of first NI? & Yes_No No \\
\hline 32 & Foley catheter present at time of first NI? & Yes_ No \\
\hline 33 & Any nurse:patient ratio $>2: 1$ on or within 48 hours preceding first NI? & Yes_No__ \\
\hline 34 & TISS score on day of fist NI & \\
\hline 35 & $\begin{array}{l}\text { Did patient receive any blood products on or within } 48 \text { hours } \\
\text { preceding first NI? }\end{array}$ & Yes__ No __ \\
\hline
\end{tabular}


Inflammatory Proteins, Genetic Variation, and Environmental Influences on Nosocomial Infection Development in Sepsis Patients

\section{DaILy OBSERVATIONS}

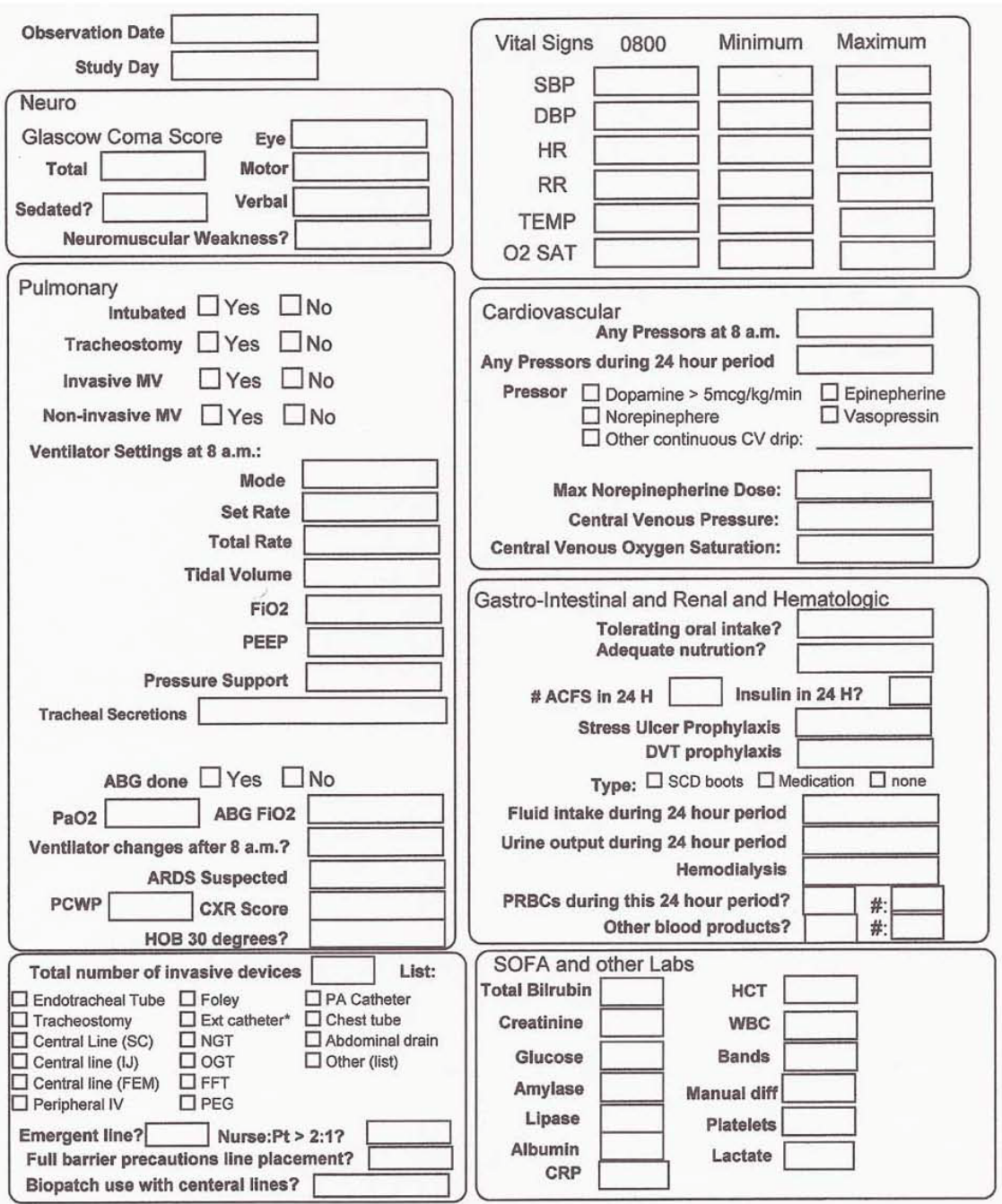


Inflammatory Proteins, Genetic Variation, and Environmental Influences on Nosocomial Infection Development in Sepsis Patients

VAMC, IRB \#, PI: Umberger

ID\#

Initials

CLINICAL PULMONARY INFECTION SCORE

Clinical Pulmonary Infection Score (CPIS Score): http://www.icumedicus.com/clinical criteria/cpis.php

\begin{tabular}{|c|c|c|c|c|c|c|c|c|}
\hline \multicolumn{3}{|c|}{ Variables } & \multicolumn{2}{|c|}{0 points } & \multicolumn{2}{|c|}{1 point } & \multicolumn{2}{|l|}{2 points } \\
\hline \multicolumn{3}{|c|}{ Tracheal Secretions } & \multicolumn{2}{|c|}{$\square$ Rare } & \multicolumn{2}{|c|}{$\square$ Abundant } & \multicolumn{2}{|c|}{$\begin{array}{l}\square \text { Abundant and } \\
\text { purulent }\end{array}$} \\
\hline \multicolumn{3}{|c|}{ Chest x-ray } & \multicolumn{2}{|c|}{$\square$ No infiltrate } & \multirow{2}{*}{\multicolumn{2}{|c|}{$\square$ Diffuse infiltrate }} & \multicolumn{2}{|c|}{$\square$ Focal Infiltrate } \\
\hline \multicolumn{3}{|c|}{ Temperature (C) } & \multicolumn{2}{|c|}{$\square \mathbf{3 6 . 0 - 3 8 . 4}$} & & $\square$ 38.5-38.9 & \multicolumn{2}{|c|}{$\square<36$ or $>39$} \\
\hline \multicolumn{3}{|c|}{ WBC } & \multicolumn{2}{|c|}{$\square$ 4-11 } & \multicolumn{2}{|c|}{$\square<4$ or $>11$} & \multicolumn{2}{|c|}{$\begin{array}{l}\square<4 \text { or }>11 \text { with } \\
\text { bands }>0.5\end{array}$} \\
\hline \multicolumn{3}{|c|}{$\mathrm{PaO} 2 / \mathrm{FiO} 2$} & \multicolumn{2}{|c|}{$\square>240$ or ARDS } & \multicolumn{2}{|c|}{$\ldots$} & \multicolumn{2}{|c|}{$\square<240$ without ARDS } \\
\hline \multicolumn{3}{|c|}{ Microbiology } & \multicolumn{2}{|c|}{$\square$ Negative } & \multicolumn{2}{|c|}{$\ldots$} & $\square$ Positiv & \\
\hline Day & Date & Sec & tions & CXR & Temp (C) & WBC & $\mathrm{PaO} 2 / \mathrm{FiO} 2$ & Microbiology \\
\hline 1 & & & & & & & & \\
\hline 2 & & & & & & & & \\
\hline 3 & & & & & & & & \\
\hline 4 & & & & & & & & \\
\hline 5 & & & & & & & & \\
\hline 6 & & & & & & & & \\
\hline 7 & & & & & & & & \\
\hline 8 & & & & & & & & \\
\hline 9 & & & & & & & & \\
\hline 10 & & & & & & & & \\
\hline 11 & & & & & & & & \\
\hline 12 & & & & & & & & \\
\hline 13 & & & & & & & & \\
\hline 14 & & & & & & & & \\
\hline 15 & & & & & & & & \\
\hline 16 & & & & & & & & \\
\hline 17 & & & & & & & & \\
\hline 18 & & & & & & & & \\
\hline 19 & & & & & & & & \\
\hline 20 & & & & & & & & \\
\hline 21 & & & & & & & & \\
\hline 22 & & & & & & & & \\
\hline 23 & & & & & & & & \\
\hline 24 & & & & & & & & \\
\hline 25 & & & & & & & & \\
\hline 26 & & & & & & & & \\
\hline 27 & & & & & & & & \\
\hline 28 & & & & & & & & \\
\hline
\end{tabular}


Inflammatory Proteins, Genetic Variation, and Environmental Influences on Nosocomial Infection Development in Sepsis Patients

VAMC, IRB \#, PI: Umberger

ID\#

Initials

INVASIVE DEVICES

\begin{tabular}{|c|c|c|c|c|c|c|c|c|c|c|c|c|c|c|c|c|c|c|c|}
\hline Day & Date & $\begin{array}{l}\frac{5}{w} \\
\frac{\tilde{W}}{0} \\
\overline{0}\end{array}$ & 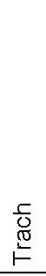 & $\begin{array}{l}\frac{5}{1} \\
\frac{1}{0} \\
0 \\
\frac{5}{2} \\
\frac{1}{Z}\end{array}$ & $\begin{array}{l}\text { U. } \\
\underline{\Lambda}\end{array}$ & 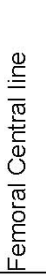 & 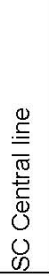 & 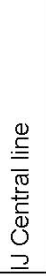 & 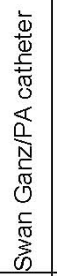 & 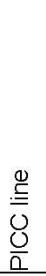 & 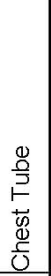 & 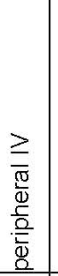 & $\begin{array}{l}\geq \\
\frac{\bar{\sigma}}{\omega} \\
\frac{\overline{0}}{\frac{0}{2}} \\
\frac{\bar{Q}}{\alpha}\end{array}$ & 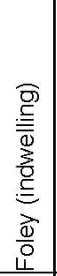 & 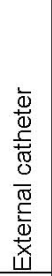 & $\begin{array}{l}\frac{.5}{\overline{0}} \\
\frac{10}{0}\end{array}$ & $\begin{array}{l}\overline{\mathbf{D}} \\
\underline{ \pm} \\
\end{array}$ & $\begin{array}{l}\frac{\grave{\Phi}}{\underline{t}} \\
\end{array}$ & 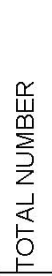 \\
\hline 1 & & & & & & & & & & & & & & & & & & & \\
\hline 2 & & & & & & & & & & & & & & & & & & & \\
\hline 3 & & & & & & & & & & & & & & & & & & & \\
\hline 4 & & & & & & & & & & & & & & & & & & & \\
\hline 5 & & & & & & & & & & & & & & & & & & & \\
\hline 6 & & & & & & & & & & & & & & & & & & & \\
\hline 7 & & & & & & & & & & & & & & & & & & & \\
\hline 8 & & & & & & & & & & & & & & & & & & & \\
\hline 9 & & & & & & & & & & & & & & & & & & & \\
\hline 10 & & & & & & & & & & & & & & & & & & & \\
\hline 11 & & & & & & & & & & & & & & & & & & & \\
\hline 12 & & & & & & & & & & & & & & & & & & & \\
\hline 13 & & & & & & & & & & & & & & & & & & & \\
\hline 14 & & & & & & & & & & & & & & & & & & & \\
\hline 15 & & & & & & & & & & & & & & & & & & & \\
\hline 16 & & & & & & & & & & & & & & & & & & & \\
\hline 17 & & & & & & & & & & & & & & & & & & & \\
\hline 18 & & & & & & & & & & & & & & & & & & & \\
\hline 19 & & & & & & & & & & & & & & & & & & & \\
\hline 20 & & & & & & & & & & & & & & & & & & & \\
\hline 21 & & & & & & & & & & & & & & & & & & & \\
\hline 22 & & & & & & & & & & & & & & & & & & & \\
\hline 23 & & & & & & & & & & & & & & & & & & & \\
\hline 24 & & & & & & & & & & & & & & & & & & & \\
\hline 25 & & & & & & & & & & & & & & & & & & & \\
\hline 26 & & & & & & & & & & & & & & & & & & & \\
\hline 27 & & & & & & & & & & & & & & & & & & & \\
\hline 28 & & & & & & & & & & & & & & & & & & & \\
\hline
\end{tabular}


Inflammatory Proteins, Genetic Variation, and Environmental Influences on Nosocomial Infection Development in Sepsis Patients

VAMC, IRB \#, PI: Umberger

ID\#

Initials

THERAPEUTTC INTERVENTION SCORING SYSTEM-28

\begin{tabular}{|c|c|c|c|c|c|c|c|c|c|}
\hline \multirow{2}{*}{\multicolumn{2}{|c|}{ CARDIOVASCULAR }} & \multirow[b]{2}{*}{ Points } & \multirow[t]{2}{*}{ Date } & \multirow[t]{2}{*}{ Date } & \multirow[t]{2}{*}{ Date } & \multirow[t]{2}{*}{ Date } & \multirow[t]{2}{*}{ Date } & \multirow[t]{2}{*}{ Date } & \multirow[t]{2}{*}{ Date } \\
\hline & & & & & & & & & \\
\hline C1 & $\begin{array}{l}\text { Cardiac arrest and/or counter shock } \\
\text { within past } 24 \text { hours }\end{array}$ & 3 & & & & & & & \\
\hline $\mathrm{C} 2$ or & $\begin{array}{l}\text { Single vasoactive drug EXCLUDING } \\
\text { dopamine } \leq 4 \mathrm{mcg} / \mathrm{kg} / \mathrm{min}\end{array}$ & 3 & & & & & & & \\
\hline C3 & $\begin{array}{l}\text { Multiple vasoactive drugs } \\
\text { EXCLUDING dopamine } \leq 4 \\
\mathrm{mcg} / \mathrm{kg} / \mathrm{min}\end{array}$ & 4 & & & & & & & \\
\hline $\mathrm{C} 4$ & $\begin{array}{l}\text { IV replacement of large fluid losses (> } \\
5 \mathrm{~L} / \text { day) }\end{array}$ & 4 & & & & & & & \\
\hline $\mathrm{C5}$ & Arterial catheter & 5 & & & & & & & \\
\hline C6 & PA catheter & 8 & & & & & & & \\
\hline $\mathrm{C7}$ & Central line (SL, DL, TLC) & 2 & & & & & & & \\
\hline
\end{tabular}

VENTILATORY

\begin{tabular}{|l|l|c|l|l|l|l|l|l|l|}
\hline V1 or & Mechanical ventilation & 5 & & & & & & & \\
\hline V2 & $\begin{array}{l}\text { Other oxygen support (NOT supplied } \\
\text { by MV) }\end{array}$ & 2 & & & & & & & \\
\hline V3 & ETT or trach care & 1 & & & & & & & \\
\hline V4 & $\begin{array}{l}\text { Chest percussion, aerosol tx, } \\
\text { incentive spirometry, NT suctioning }\end{array}$ & 1 & & & & & & & \\
\hline
\end{tabular}

RENAL (Include wtikg_and TOTAL furosemide dose/day

\begin{tabular}{|l|l|c|l|l|l|l|l|l|l|}
\hline R1 & CAVH & 3 & & & & & & & \\
\hline R2 & Urine output $\leq \mathrm{q} 2 \mathrm{~h}$ & 2 & & & & & & & \\
\hline R3 & $\begin{array}{l}\text { Active diuresis: furosemide } \geq 0.5 \\
\text { mg/kg/day }\end{array}$ & 3 & & & & & & & \\
\hline
\end{tabular}
NEUROLOGIC

\begin{tabular}{|l|l|l|l|l|l|l|l|l|l|}
\hline N1 & Monitoring of ICP & 4 & & & & & & & \\
\hline
\end{tabular} METABOLIC

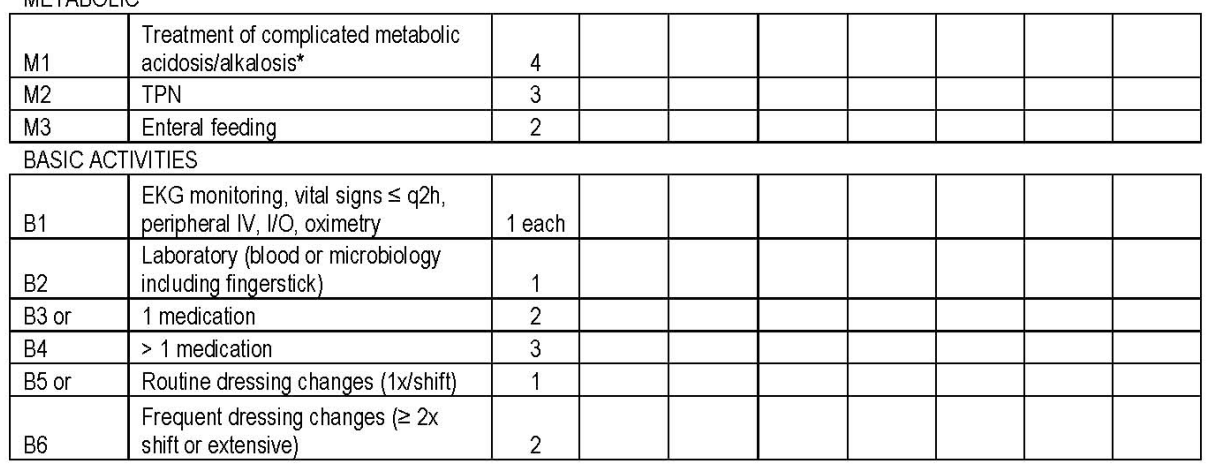


Inflammatory Proteins, Genetic Variation, and Environmental Influences on Nosocomial Infection Development in Sepsis Patients

VAMC, IRB \#, PI: Umberger

ID\# Initials

\begin{tabular}{l|l|l|l|l|l|l|l|l|l|} 
B7 & $\begin{array}{l}\text { Care of drains/chest tubes except } \\
\text { NGT or Foley catheter }\end{array}$ & 3 & & & & & & \\
\hline TISS & Page 2 \\
SPECIFIC INTERVENTIONS & $\begin{array}{l}\text { Single procedure/intervention in the } \\
\text { ICU* }\end{array}$ & 3 & & & & & & & \\
\hline S1 & $>1$ procedure/intervention in the ICU & 5 & & & & & & & \\
\hline S2 & Procedure requiring transport off unit & 5 & & & & & & & Date \\
\hline S3
\end{tabular}

OTHERS

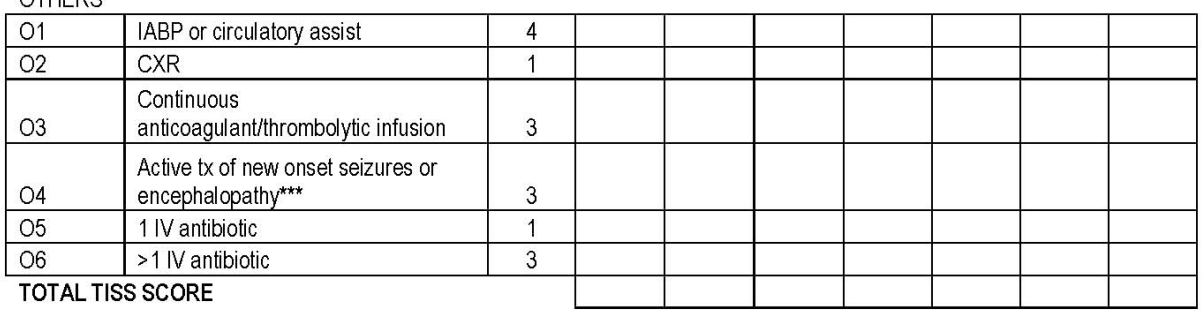

*Insulin drip for DKA/HHNK, bicarb drip, > 2 amps bicarb in 24 hours, ammonium chl oride drip

**Intubation, line insertion, chest tube insertion, endoscopy,

etc.

***Lactulose/kayexalate enemas 
Inflammatory Proteins, Genetic Variation, and Environmental Influences on Nosocomial Infection Development in Sepsis Patients

VAMC, IRB \#, PI: Umberger

ID\# Initials

INFECTION REPORT

All cultures drawn are to be recorded. Culture results are to be reviewed by investigators along with sensitivity reports and antibiotics.

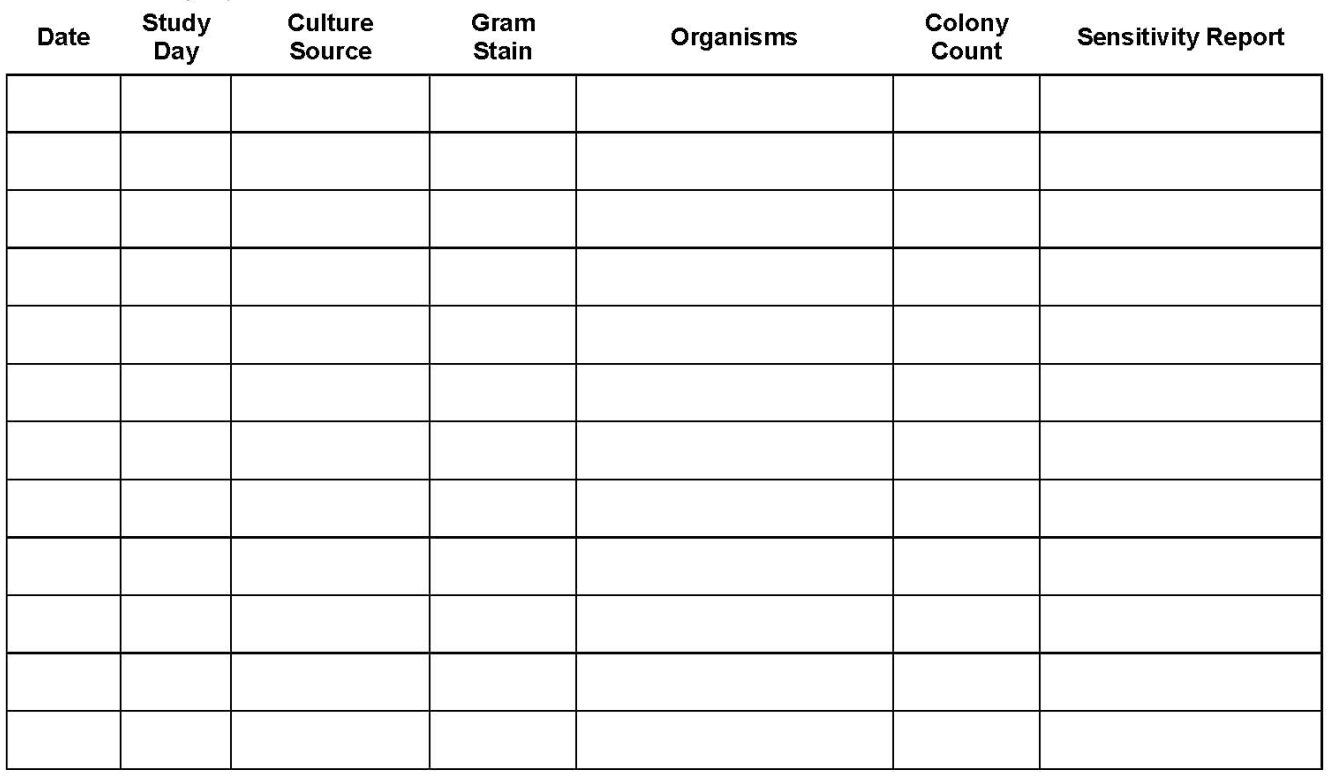

Nosocomial Infections

\begin{tabular}{|c|c|c|c|c|c|c|c|}
\hline Date & $\begin{array}{c}\text { Site of } \\
\text { Infection }\end{array}$ & Organism & $\begin{array}{c}\text { Type of } \\
\text { Organism }\end{array}$ & $\begin{array}{c}\text { Final Infection } \\
\text { Diagnosis }\end{array}$ & $\begin{array}{l}\text { Definitive } \\
\text { Infection? }\end{array}$ & $\begin{array}{l}\text { Fever with } \\
\text { Infection? }\end{array}$ & $\begin{array}{l}\text { Appropriate } \\
\text { antibiotics? }\end{array}$ \\
\hline & & & & & & & \\
\hline & & & & & & & \\
\hline & & & & & & & \\
\hline & & & & & & & \\
\hline & & & & & & & \\
\hline
\end{tabular}


Inflammatory Proteins, Genetic Variation, and Environmental Influences on Nosocomial Infection Development in Sepsis Patients

VAMC, IRB \#, PI: Umberger

ID\# Initials

CONCURRENT INPATIENT MEDICATIONS-ANTIBIOTICS

$\square$ Check here if no medications.

List all medications ( 3 days prior to ICU admission to ICU discharge (up to 28 days))

\begin{tabular}{|c|c|c|c|c|c|c|c|}
\hline & Trade/Generic Name & Reason for use & Dose & Route & Start date & $\begin{array}{l}\text { Stop } \\
\text { Date }\end{array}$ & $\begin{array}{c}\text { Ongoing } \\
\text { Y or N }\end{array}$ \\
\hline 1 & & & & & & & \\
\hline 2 & & & & & & & \\
\hline 3 & & & & & & & \\
\hline 4 & & & & & & & \\
\hline 5 & & & & & & & \\
\hline 6 & & & & & & & \\
\hline 7 & & & & & & & \\
\hline 8 & & & & & & & \\
\hline 9 & & & & & & & \\
\hline 10 & & & & & & & \\
\hline 11 & & & & & & & \\
\hline 12 & & & & & & & \\
\hline 13 & & & & & & & \\
\hline 14 & & & & & & & \\
\hline 15 & & & & & & & \\
\hline 16 & & & & & & & \\
\hline 17 & & & & & & & \\
\hline 18 & & & & & & & \\
\hline 19 & & & & & & & \\
\hline 20 & & & & & & & \\
\hline
\end{tabular}


Inflammatory Proteins, Genetic Variation, and Environmental Influences on Nosocomial Infection Development in Sepsis Patients

VAMC, IRB \#, PI: Umberger

ID\# Initials

Concurrent Inpatient Medications-Other Mediations

Check here if no medications.

List all medications ( 3 days prior to ICU admission to ICU discharge (up to 28 days))

\begin{tabular}{|c|c|c|c|c|c|c|c|}
\hline & Trade/Generic Name & Reason for use & Dose & Route & Start date & $\begin{array}{l}\text { Stop } \\
\text { Date }\end{array}$ & $\begin{array}{l}\text { Ongoing } \\
\mathrm{Y} \text { or } \mathrm{N}\end{array}$ \\
\hline 1 & & & & & & & \\
\hline 2 & & & & & & & \\
\hline 3 & & & & & & & \\
\hline 4 & & & & & & & \\
\hline 5 & & & & & & & \\
\hline 6 & & & & & & & \\
\hline 7 & & & & & & & \\
\hline 8 & & & & & & & \\
\hline 9 & & & & & & & \\
\hline 10 & & & & & & & \\
\hline 11 & & & & & & & \\
\hline 12 & & & & & & & \\
\hline 13 & & & & & & & \\
\hline 14 & & & & & & & \\
\hline 15 & & & & & & & \\
\hline 16 & & & & & & & \\
\hline 17 & & & & & & & \\
\hline 18 & & & & & & & \\
\hline 19 & & & & & & & \\
\hline 20 & & & & & & & \\
\hline
\end{tabular}


Inflammatory Proteins, Genetic Variation, and Environmental Influences on Nosocomial Infection Development in Sepsis Patients

VAMC, IRB \#, PI: Umberger

ID\# Initials

SPECIAL Procedures

\begin{tabular}{|l|l|l|l|l|}
\hline \multicolumn{1}{c}{ Date } & \multicolumn{1}{c}{ Study Day } & \multicolumn{1}{l}{ Type of Procedure } & Reason for Procedure & \multicolumn{1}{l}{ Procedure Report Obtained } \\
\hline & & & & \\
\hline & & & & \\
\hline & & & & \\
\hline & & & & \\
\hline & & & & \\
\hline & & & & \\
\hline & & & & \\
\hline & & & & \\
\hline & & & & \\
\hline & & & & \\
\hline & & & & \\
\hline & & & & \\
\hline & & & & \\
\hline & & & & \\
\hline & & & & \\
\hline & & & & \\
\hline & & & & \\
\hline & & & & \\
\hline & & & & \\
\hline & & & & \\
\hline & & & & \\
\hline & & & & \\
\hline
\end{tabular}


Inflammatory Proteins, Genetic Variation, and Environmental Influences on Nosocomial Infection Development in Sepsis Patients

VAMC, IRB \#, PI: Umberger

ID\# Initials

STUDY NOTES

Record conversations related to study decisions here. Include discussion related to any adverse events as well as general comments as needed. Include contacts made with the patient/family.

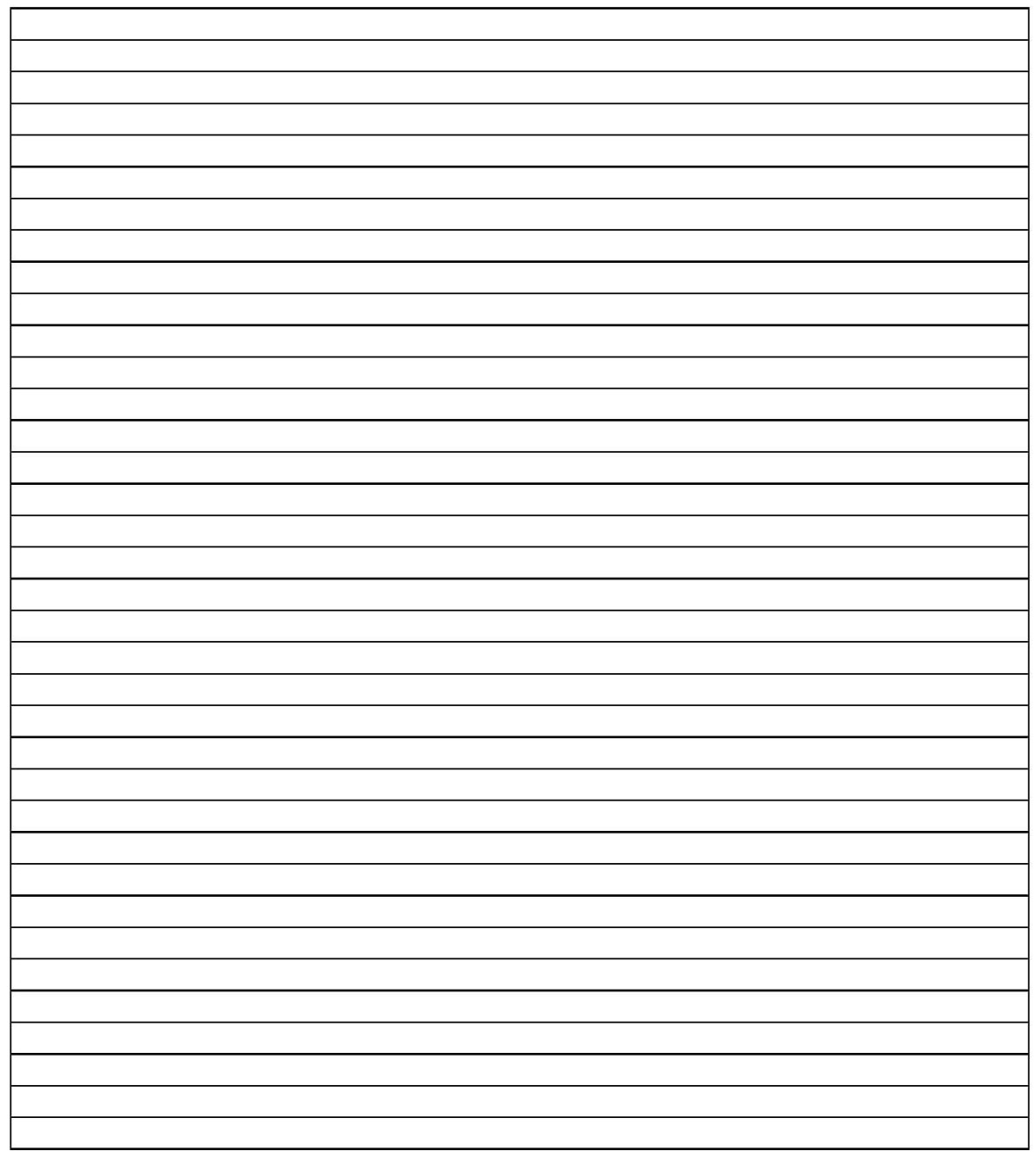




\section{VITA}

Reba Antionette Umberger was born in Memphis, TN in 1966. After graduating from Olive Branch High School, she attended Northwest Mississippi Junior College and received the degree of Associate of Science in Nursing in 1987. Subsequently she entered the University of Memphis and earned a Bachelor of Science degree in biology (microbiology) in 1994. She later pursued graduate education at the University of Tennessee Health Science in 2001 and enrolled in the Master of Science program in epidemiology, earning the M.S. degree in 2004. Reba began doctoral studies in 2006 and will graduate with a Doctor of Philosophy in Nursing from The University of Tennessee Health Science Center in May 2011. She completed a fellowship in genetics at the National Institutes of Health (2008). She has worked in critical care for more than 20 years, at the bedside and in research, and currently maintains CCRN certification. 